Aus der Poliklinik für Kieferorthopädie

(Direktor: Prof. Dr. med. dent. D. Kubein-Meesenburg)

im Zentrum Zahn-, Mund- und Kieferheilkunde

der Medizinischen Fakultät der Universität Göttingen

Ausmaß und Beständigkeit der ästhetischen Verbesserung von Multibrackettherapie-induzierten White-Spot-Läsionen nach Icon-Infiltration -eine prospektive, randomisierte, splitmouth-kontrollierte klinische Studie

\author{
Inaugural-Dissertation \\ zur Erlangung des Doktorgrades \\ für Zahnheilkunde \\ der Medizinischen Fakultät der \\ Georg-August-Universität zu Göttingen
}

vorgelegt von

Amely Eckstein

aus

Bremen

Göttingen 2013 
Dekan: $\quad$ Prof. Dr. rer. nat. H. Kroemer

1. Berichterstatter: PD Dr. med. dent. Michael Knösel

2. Berichterstatter: PD Dr. med. dent. Tina Rödig

Tag der mündlichen Prüfung: $\quad 30.04 .2014$ 


\section{Inhaltsverzeichnis}

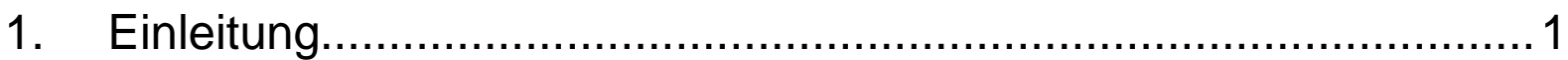

2. Literaturübersicht................................................................ 3

2.1. Makroskopische Anatomie des Schmelzes …….................................... 3

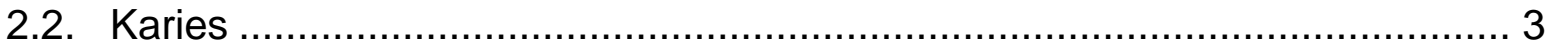

2.3. White-Spot-Läsionen (WSL) - Allgemein .............................................. 4

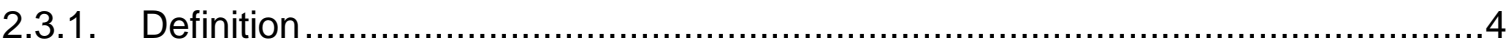

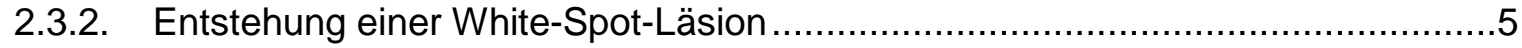

2.3.3. Stellenwert von Streptococcus mutans bei der Kariesentstehung .......................8

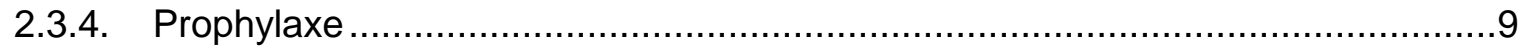

2.3.5. White-Spot-Läsionen nach orthodontischer Behandlung ...................................11

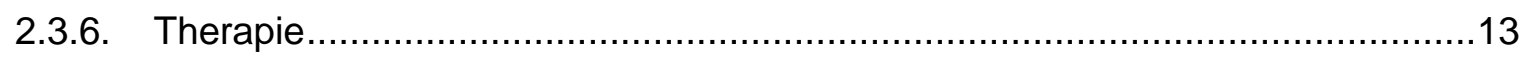

3. Ziele der Arbeit ................................................................... 17

4. Material und Methode ........................................................... 18

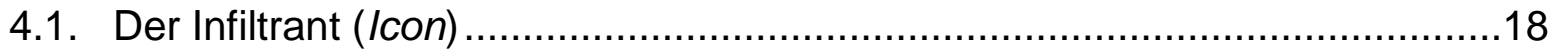

4.2. Dentale Farb- und Helligkeitsbestimmung ..............................................19

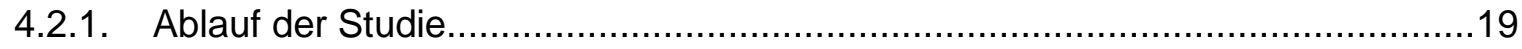

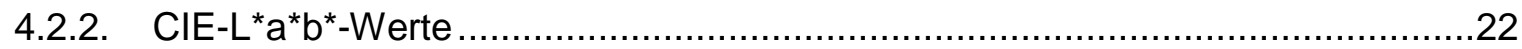

4.2.3. Dentale Farbbestimmung: Technische Problemstellungen ................................23

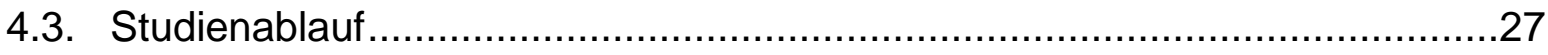

4.3.1. Behandlungsablauf .................................................................................

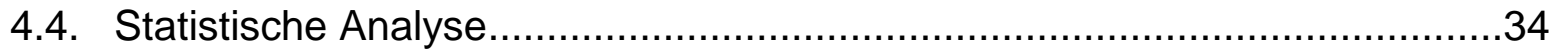

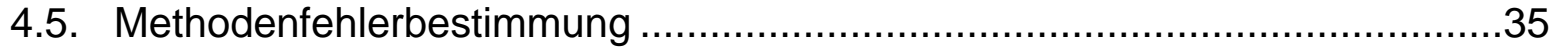

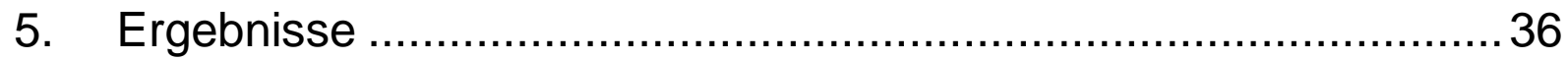

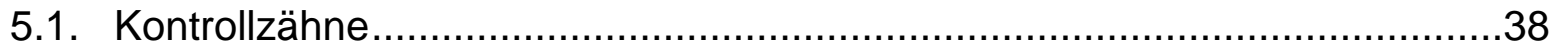

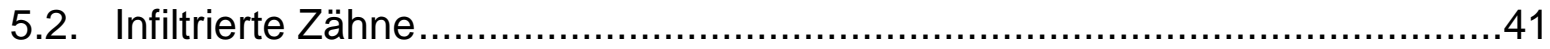

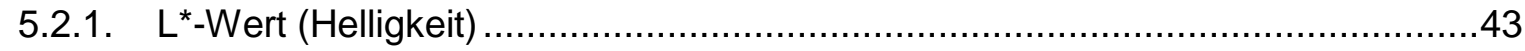

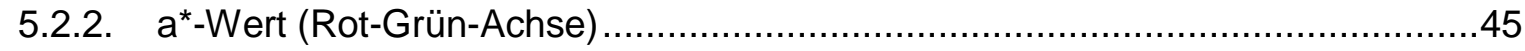

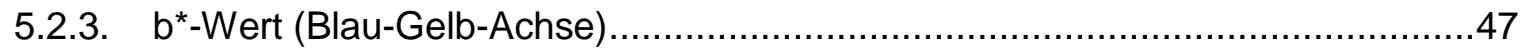

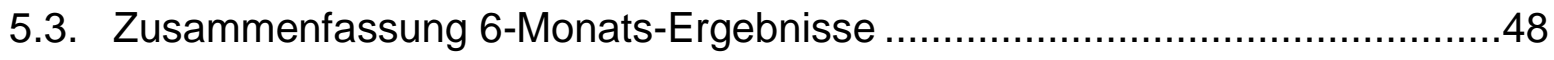

5.4. Nachuntersuchung der infiltrierten Zähne nach 12 Monaten .......................49 


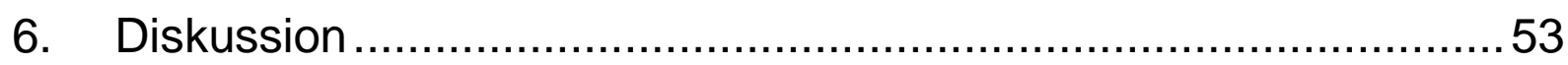

6.1. Problemstellungen bei der WSL-Behandlung ...................................53

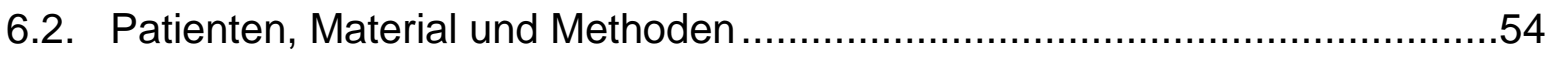

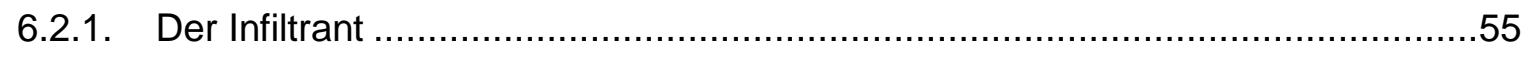

6.2.2. Anrauen des umliegenden Schmelzes .......................................................56

6.2.3. Der Faktor der Läsionstiefe, -ausdehnung und -alter der WSL ........................57

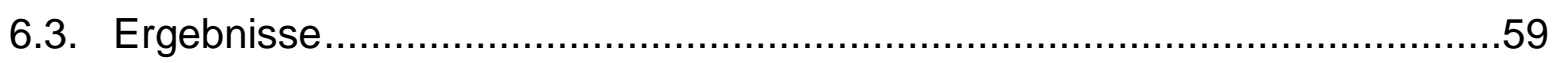

6.3.1. Kontrollierte Farb- und Helligkeitsentwicklung über 6 Monate ..........................59

6.3.2. Baselinekontrollierte Farb- und Helligkeitsentwicklung über 12 Monate ............59

6.3.3. Zusammengefasste Farb- und Helligkeitsentwicklung ..................................60

6.4. Perspektiven für weitergehende Forschung ...................................60

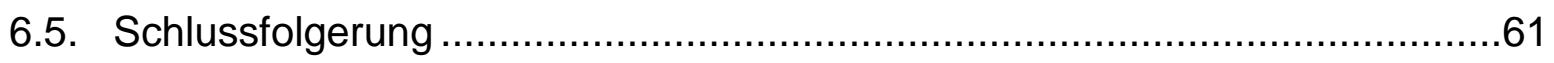

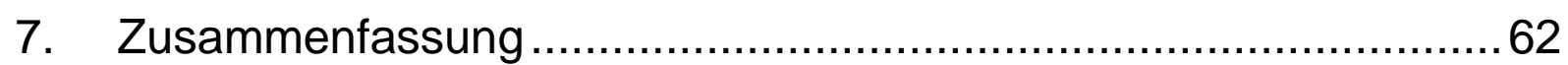

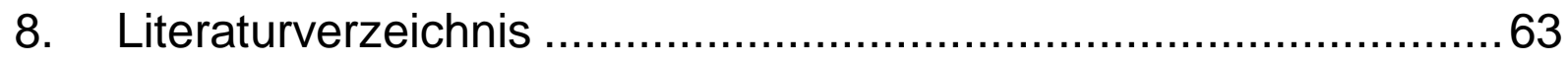

9. Abkürzungsverzeichnis............................................................

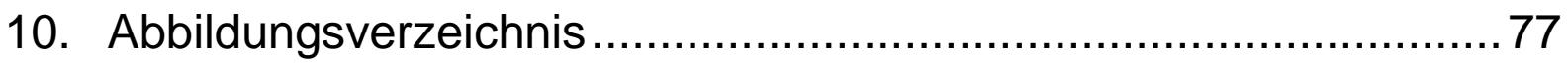

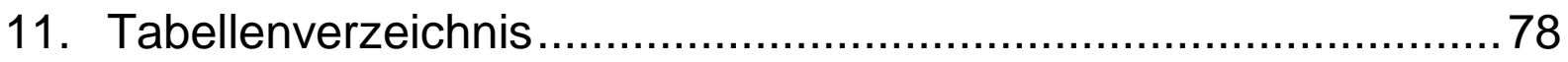

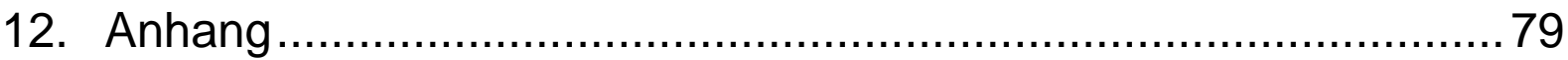




\section{Einleitung}

Im Rahmen einer kieferorthopädischen Behandlung werden dental und basal bedingte Dysgnathien mit dem Ziel behandelt, eine funktionell optimale und gesicherte Okklusion herzustellen und gleichzeitig den gestiegenen Ansprüchen an eine harmonische dentofaziale Ästhetik nachzukommen (Buren et al. 2008). Die heutige Kieferorthopädie ist ohne den Einsatz festsitzender kieferorthopädischer Apparaturen zur effizienten, gezielten Zahnbewegung und Ausformung der Zahnbögen nicht vorstellbar. Während moderne festsitzende Apparaturen die kieferorthopädische Breitenversorgung überhaupt erst möglich gemacht haben (Andrews 1979), ist der Nachteil dieser Herangehensweise jedoch in der vorübergehend erschwerten Mundhygiene zu sehen: Brackets und Bänder stellen Hygienehindernisse dar und begünstigen eine vermehrte Plaqueakkumulation, sodass ein erhöhtes Schmelz-Demineralisationsrisiko während der Behandlung besteht (Zimmer und Rottwinkel 2004, Hadler-Olsen et al. 2011). So können im Rahmen kieferorthopädischer Behandlungen in Verbindung mit inadäquater Mundhygiene schon innerhalb eines kurzen Zeitraums von nur wenigen Wochen initiale Demineralisationserscheinungen des Schmelzes - White-Spot-Läsionen - auftreten (O'Reilly und Featherstone 1987, Gorton und Featherstone 2003). Diese Läsionen sind durch eine vom gesunden Schmelz verschiedene Lichtbrechung klinisch sichtbar und trotz einer bürstabrasionsbedingten Verminderung innerhalb der ersten 6 Monate nach Entfernen der Apparatur (Debonding) oftmals irreversibel (Backer Dirks 1966, Fejerskov et al. 2003, Holmen et al. 1987).

Bei andauernder Plaqueakkumulation kann es über die bestehende ästhetische Beeinträchtigung hinaus zu einem Fortschreiten des initialen Demineralisationsprozesses und damit zu einer Vertiefung der Läsion kommen, die in der Folge eine restaurative Behandlungsnotwendigkeit mit sich bringt (Stahl und Zandona 2007). Diese Erkenntnisse implizieren, dass der Mundhygiene und der Prophylaxe im Rahmen kieferorthopädischer Behandlungen ganz besondere Beachtung geschenkt werden sollte, um das Demineralisationsrisiko zu reduzieren und somit auch aus kariespräventiver und ästhetischer Sicht ein optimales Behandlungsergebnis zu erzielen (Tufekci et al. 2011).

Obwohl die WSL-Prophylaxemaßnahmen wie Mundspülen mit niedrigkonzentrierten Fluoridlösungen und Remotivierungsmaßnahmen für den Bereich der häuslichen Zahnpflege inzwischen ein fester Bestandteil moderner kieferorthopädischer Konzepte sind, stellen WSL noch immer eine gängige Begleiterscheinung festsitzender Behandlungen dar (Buschang et al. 2013), deren Behandlung nicht einfach ist. 
Während im Falle von eingebrochenen kariösen Läsionen Restaurationen unabdingbar sind, sollten die noch nicht eingebrochenen kariösen Läsionen mit dem Therapieziel, diese zu stoppen oder zu remineralisieren, behandelt werden (Stahl und Zandona 2007).

Die klassischen Herangehensweisen zur WSL-Therapie lassen sich also in non-invasive (konservierende) und invasive Maßnahmen gliedern. Non-invasive Maßnahmen beinhalten das Remineralisieren des Schmelzes durch Anwendung lokaler Fluoride (Tezel et al. 2002, Øgaard et al. 2001, Kleber et al. 1999) sowie die begleitende Abrasion durch das Zahnbürsten (Holmen et al. 1987). Auch wenn somit Läsionen inaktiviert werden, bleibt der optisch nachteilige Effekt: Die WSL bleiben klinisch sichtbar (Gugnani 2012, Mattousch et al. 2007). Alternativ kann eine invasive Therapie erfolgen, bei der das kariogene Gewebe entfernt und durch Composite-Material im Sinne einer Füllung ersetzt wird. Trotz einer minimalinvasiven Zielsetzung (Vila Verde et al. 2009) müssen für diese Art der restaurativen Therapie immer auch wesentliche Mengen des umliegenden Gewebes entfernt werden, um alle kariösen Stellen zu erreichen und zu entfernen.

Ein vergleichsweise neuer, substanzschonender Ansatz bietet sich in Form der Karies- bzw. Läsionsinfiltration mit niedrigviskösen, lichthärtenden Kunststoffen (Infiltranten) (Paris et al. 2010). Die WSL-Infiltration als Therapiemöglichkeit wird als Brückenschlag zwischen nichtinvasiven und invasiven Behandlungsmöglichkeiten angesehen (Paris und Meyer-Lueckel 2010), da hierbei keine Entfernung des Läsionsgewebes erfolgt, also die Schmelzsubstanz erhalten bleibt. Klinische Studien (Meyer-Lueckel und Paris 2008a und b) zeigen, dass die Progression initialer, oberflächlich nicht eingebrochener Läsionen durch Infiltration aufgehalten werden kann. Ein weiterer positiver Nebeneffekt der WSL-Infiltration ist, dass durch die veränderte Lichtbrechung der infiltrierten Läsion diese optisch vom gesunden, angrenzenden Schmelz schwieriger oder gar nicht zu unterscheiden ist, was seine Ursache in dem sehr ähnlichen Lichtbrechungsindex von Infiltrant und gesundem Schmelz hat (siehe Abbildung 1b, Seite 8) (Paris und Meyer-Lueckel 2009). Neben dem kariesprotektiven Effekt ist diese farbangleichende Behandlungsmethode für den Fachbereich Kieferorthopädie von großer Bedeutung, da gerade die Frontzahnästhetik für die entsprechende Patientengruppe von sehr hohem Stellenwert ist. Es liegen derzeit keine In-vivo-Langzeituntersuchungen über die Beständigkeit des Farb- und Helligkeitsangleichs vor. Diese Informationslücke bildet den Ausgangspunkt der vorliegenden Arbeit. 


\section{Literaturübersicht}

\subsection{Makroskopische Anatomie des Schmelzes}

Die äußere Ummantelung eines Zahnes besteht im Bereich der Krone aus Schmelz, im Bereich der Wurzel aus Zement. Unter diesen Substanzen liegt das Dentin, welches direkt an die Pulpa angrenzt. Zahnschmelz ist mit $250 \mathrm{KHN}$ - $390 \mathrm{KHN}$ härter als Knochen (30 - 50 $\mathrm{KHN}$ ) und damit die härteste Substanz des menschlichen Körpers (Hellwig et al. 2009). Er besteht aus 93 - 98\% anorganischen Bestandteilen wie Kalzium, Phosphor, Karbonat, Natrium, Magnesium und weiteren Spurenelementen. Nur ein bis vier Prozent sind Wasser und der Rest organische Substanzen wie Proteine und Lipide. Gebildet wird der Zahnschmelz präeruptiv von Ameloblasten. Posteruptiv ist er für Reparaturvorgänge auf die Aufnahme von Kalzium und Phosphat aus dem Speichel bzw. aus der Nahrung angewiesen, weil die Zahnhartsubstanzen säurelöslich sind.

Die Ameloblasten bilden eine Matrix, die sich zu sogenannten Apatitkristallen kristallisiert. Um die Kristalle liegt eine Hydrationsschale, umgeben von Lipiden und Proteinen. Bis zu 100 dieser hexagonal-stäbchenförmigen Schmelzkristalle lagern sich $\mathrm{zu}$ sogenannten Schmelzprismen zusammen, die wellenförmig, stäbchenartig vom Dentin bis zur Oberfläche des Schmelzes reichen. Um diese Schmelzprismen herum liegt die zwischenprismatische Substanz. Sie besteht aus diffus angeordneten Schmelzkristallen. Die äußerste Schicht des Zahnschmelzes ist ca. 20-30 $\mu \mathrm{m}$ dick. In dieser Schicht finden sich keine Prismen, sondern nur dicht aneinander gelagerte Kristalle (Hellwig et al. 2009).

\subsection{Karies}

Die Fédération Dentaire Internationale (FDI) definiert die Zahnkaries als „lokalisierte(n), pathologische(n) Vorgang (...) bakteriellen Ursprungs (...), der zur Entmineralisierung der Zahnhartsubstanzen und schließlich zur Kavitätenbildung führt“ (Franke und Baume 1976, S.77). Karies ist eine weltweit verbreitete, unspezifische Infektionskrankheit der Zahnhartsubstanzen, die sich in unterschiedlichen Ausprägungsformen manifestiert. Initial handelt es sich um eine klinisch nicht erkennbare submikroskopische Veränderung, die sich bei Fortschreiten zu einer deutlich diagnostizierbaren Kavitation entwickelt (Hellwig et al. 2009). 


\subsection{White-Spot-Läsionen (WSL) - Allgemein}

\subsubsection{Definition}

White-Spot-Läsionen sind weißlich-opake Entmineralisationen der Schmelzoberfläche mit glatter bis leicht angerauter Oberfläche. Ätiologisch gesehen bezeichnet man diese auch als Schmelz-Demineralisationen, die eine typische Folge einer unzureichenden Mundhygiene sind. Das heißt, es handelt sich um eine Karies-Vorstufe, entstanden unter einer über Wochen einwirkenden Schicht aus Zahnbelag und Plaque (Hellwig et al. 2009). Durch die Demineralisation in diesen Arealen bilden sich im Schmelz Diffusionswege für Säuren und Mineralien. Bei ausbleibender Behandlung der WSL und anhaltend schlechter Mundhygiene kann sich eine Karies manifestieren, die entsprechend aufwändig mit einer Füllung therapiert werden muss. Darüber hinaus stellen WSL für die Patienten neben der kariogen bedingten Veränderung auch eine erhebliche ästhetische Beeinträchtigung dar. Vor allem im gut sichtbaren Frontzahnbereich wird dies als störend empfunden.

WSL sind eine initiale Karies. Karies ist die häufigste Erkrankung der Zahnhartsubstanzen und eine der häufigsten menschlichen Erkrankungen insgesamt (Subramaniam et al. 2005). Miller hat 1898 eine Entstehungstheorie der Karies vorgestellt, die später verifiziert und erweitert wurde. Sie besagt, dass bei einem Vorkommen von zu viel kariogenem Substrat die kariogenen Mikroorganismen in der Plaque organische Säuren produzieren. Wenn diese Säuren zu lange auf die Zahnhartsubstanz einwirken, führt das zu Entmineralisierungen (Hellwig et al. 2009). Für die Entstehung einer Demineralisation ist die Koexistenz der vier Faktoren Plaque, Substrat (speziell niedermolekulare Kohlenhydrate), eine anfällige Zahnoberfläche (Wirt) und Zeit nötig (Hellwig et al. 2009). Die Speichel-Parameter wie der ph-Wert, die Fließrate und die Pufferkapazität können den Grad der Entmineralisierung beeinflussen. Indem sie auf den Säureangriff folgend, Einfluss auf die Geschwindigkeit, mit der die Demineralisation voranschreitet, nehmen, erhöhen sie die Wahrscheinlichkeit der Remineralisation (Newbrun 1989).

Karies ist das Ergebnis eines Kreislaufs der De- und Remineralisation des Schmelzes, der durch wechselnde $\mathrm{pH}$-Levels verschuldet ist (Burne und Marquis 2000).

Der Kreislauf der De- und Remineralisation ist ein ständiges Phänomen und findet in der Mundhöhle immer im Anschluss an die Aufnahme verschiedener Mahlzeiten und Getränke statt.

Wenn niedermolekulare Kohlenhydrate aus der Nahrung in die Plaque diffundieren, werden diese dort von Mikroorganismen verstoffwechselt, wodurch es zur Bildung von Säuren und 
somit zu einem Abfall des pH-Wertes im Speichel kommt (Stephan 1940). Der für den Schmelz kritische pH-Wert liegt zwischen 5,2 und 5,7. Bei einem zu sauren pH-Wert kommt es zum Mineralverlust aus der Schmelzoberfläche. Dabei werden Mineralien aus dem Schmelzkristallgitter herausgelöst und wandern in die Plaque ab (Demineralisation). Durch den Speichel erfolgt in der Plaque eine rasche Neutralisation der Säuren. Es kommt zu einem Anstieg des $\mathrm{pH}$-Wertes sowie zu einer umgekehrten lonenbewegung von der Plaque in den Schmelz (Remineralisation).

Wird die Plaque über einen längeren Zeitraum nicht entfernt und sind die protektiven Faktoren, wie zum Beispiel der Speichelpuffer und die Fluoride, nicht mehr in der Lage, die Balance auf Seiten der Remineralisation zu halten, entsteht ein Verlust von Mineralien. Das führt zu beginnenden Kariesläsionen (Hellwig et al. 2009).

Diese sind vorerst nur mikroskopisch erkennbar, stellen bei anhaltender Demineralisation aber auch klinisch sichtbare Veränderungen dar.

Das erste Anzeichen einer beginnenden Demineralisation auf der glatten Schmelzoberfläche der Krone ist eine kreidig matte White-Spot-Läsion. Typischerweise verlieren die Bereiche der WSL aufgrund der umfangreichen Porositäten unter der Oberfläche, die durch die Demineralisation entstehen, ihre Transluzenz (Glazer 2009, Paris und Meyer-Lueckel 2009). So entwickeln sich „weißlich opake Veränderungen an der Schmelzoberfläche“, (Hellwig et al. 2009), die sich bei einer Sondenkontrolle leicht rau anfühlen können, deren Oberfläche aber intakt ist. WSL stellen nach der kieferorthopädischen Entbänderung häufig ein ästhetisches Problem dar, das auch bestehen bleibt wenn der Progress dieser Kariesläsionen durch präventive Maßnahmen wie z.B. die Fluoridierung aufgehalten werden kann. (Gugnani et al. 2012).

\subsubsection{Entstehung einer White-Spot-Läsion}

Bei der Entstehung eines White-Spots legt sich zunächst ein Schutzfilm aus adsorbierten Proteinen auf den gesunden und frisch geputzten Zahnschmelz (Plagmann 1998). Es lagern sich Fluoride an, die die Schmelzlöslichkeit reduzieren.

So schützt der Film zum einen die Schmelzoberfläche, dient aber andererseits auch Mikroorganismen als Anlagerungsfläche. Schon innerhalb von null bis zwei Tagen kann auf dem Schutzfilm durch deren Anlagerung Plaque entstehen.

Bei Zahnplaque handelt es sich um einen komplexen Biofilm aus dreidimensional organisierten Bakterienpopulationen, eingebettet in einer extrazellulären Matrix aus Polymeren (Marsh und Martin 2003). Dieser Biofilm haftet auf der Zahnoberfläche und entwickelt sich innerhalb von mehreren Tagen. Von einer ausgereiften Plaque spricht man 
erst nach 7-14 Tagen. Zu diesem Zeitpunkt hat sich bereits ein komplexes, miteinander interagierendes, System von bis zu 700 verschiedenen konkurrierenden und symbiotisch koexistierenden Bakterienspezies gebildet (Aas et al. 2005, Marsh et al. 2011).

Abhängig von der Struktur sowie von der mikrobiellen Zusammensetzung der Plaque ist deren Kariogenität (Busscher und van der Mei 1997). Das besagt auch die ökologische Plaquehypothese: Potentiell krankheitserregende Organismen können an mehreren Orten in geringer Menge vorkommen, ohne eine Krankheit zu erzeugen. Kommt es durch veränderte lokale Umweltbedingungen allerdings zu einem Anstieg von Mikroorganismen und somit zu einer Veränderung der Residentmikroflora, kann das pathologische Prozesse nach sich ziehen. So führt ein über längere Zeit abgesenkter pH-Wert in der Mundhöhle, der z.B. aus vermehrtem Zuckerkonsum resultiert, zu einer Veränderung in der Mikroflora. Es kommt zu einem Anstieg azidogener und säuretoleranter Bakterien, wodurch die Plaque ein kariogenes Potential entwickelt. Verantwortlich sind hier vor allem Streptococcus mutans und Laktobazillen. Diese können sich gut an die pH-Wert-Veränderung adaptieren und sind deshalb anderen Mikroorganismen kompetitiv überlegen (Marsh 1995, Marsh et al. 2011).

Die kariogenen Mikroorganismen produzieren aus Kohlenhydraten Säuren wie Lactat und Acetat. Diese organischen Säuren führen zu interprismatischem Mineralisationsverlust und somit zu einer frühen Läsion. Über diese interprismatischen Bereiche können die Säuren tiefer in den Zahnschmelz diffundieren und sich dort teilen. Dabei werden Wasserstoffionen frei, die das Kristallgitter angreifen, wodurch Kalzium-, Hydroxid-, Phosphat-, Fluorid-, Kobalt-, Natrium und Magnesiumionen gelöst werden. Diese diffundieren in das Wasser um die Kristalle, von dort durch die vergrößerten Poren zur Schmelzoberfläche und dann in die Plaque. Solange die Plaque Säure produziert, halten die Demineralisationsvorgänge, in denen vor allem Kalzium und Phosphat verloren gehen, an (Hellwig et al. 2009).

Bei der lichtmikroskopischen Untersuchung eines Dünnschliffs von Schmelz mit einer WSL kann man, durch verschiedene Behandlungen des Präparates, vier Zonen erkennen. (siehe Abbildung 1a, Seite 7) Die Oberfläche erscheint mikroskopisch trotz geringem Mineralverlust intakt, weshalb sie als pseudointakte Oberfläche bezeichnet wird. Das Porenvolumen, das beim gesunden Schmelz bei $0,1 \%$ liegt, beträgt hier ungefähr $5 \%$. Der absolute Mineralverlust ist hier gering, da Kalzium und Phosphat bei der Diffusion nach extern an der Oberfläche repräzipitieren und sich an bereits geschädigte Kristalle anlagern. Doch durch die pseudointakte Oberfläche können die Säuren über den Läsionskörper bis in die transluzente Zone, die letzte Zone vor dem Dentin, diffundieren und auf dem Weg zu weiteren Demineralisationsvorgängen führen. Der Läsionskörper befindet sich unter der pseudointakten Oberfläche. Hier findet der volumenmäßig höchste Mineralverlust mit einer Verminderung von bis zu $25 \%$ statt. Es ist das Gebiet mit der größten Demineralisation 
(Klimm 1997), in das auch Speichelbestandteile eindringen können. Das Porenvolumen beträgt ebenfalls bis zu 25\%. In der dritten, der dunklen Zone, ist das Porenvolumen $2-4 \%$. Die Kristalle der dunklen Zone sowie auch die der pseudointakten Oberfläche sind wegen der Remineralisierungsvorgänge größer als die des gesunden Schmelzes. An die dunkle Zone schließt sich, zum Dentin hin, die transluzente Zone an, in der die Demineralisation fortschreitet, die Poren entstehen und sich vergrößern. Das Porenvolumen beträgt in diesem Bereich durchschnittlich 1\% (Hellwig et al. 2009). Aufgrund des Mineralverlusts sind die Kristalle in der transluzenten Zone und im Läsionskörper kleiner als die Kristalle des gesunden Schmelzes. Wird die Plaque nicht entfernt, so vergrößert sich die Läsion weiter und geht ins Dentin über. Eine Schmelzläsion kann aber, wenn man die kariogenen Noxen entfernt und entsprechende Prophylaxemaßnahmen - wie Fluoridierung - einleitet, zum Stillstand kommen oder sogar remineralisieren (Hellwig et al. 2009).
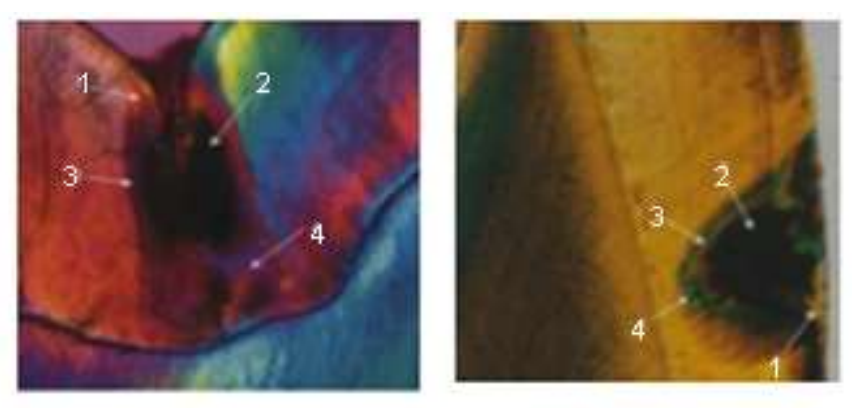

Abbildung 1a: Polarisationsmikroskopisches Bild einer Schmelzkaries: 1) pseudointakte Oberflächenschicht, 2) Zentrum der Läsion, 3) dunkle Zone, 4) transluzente Zone (Abbildung nach Gängler et al. 2010, S.120) 


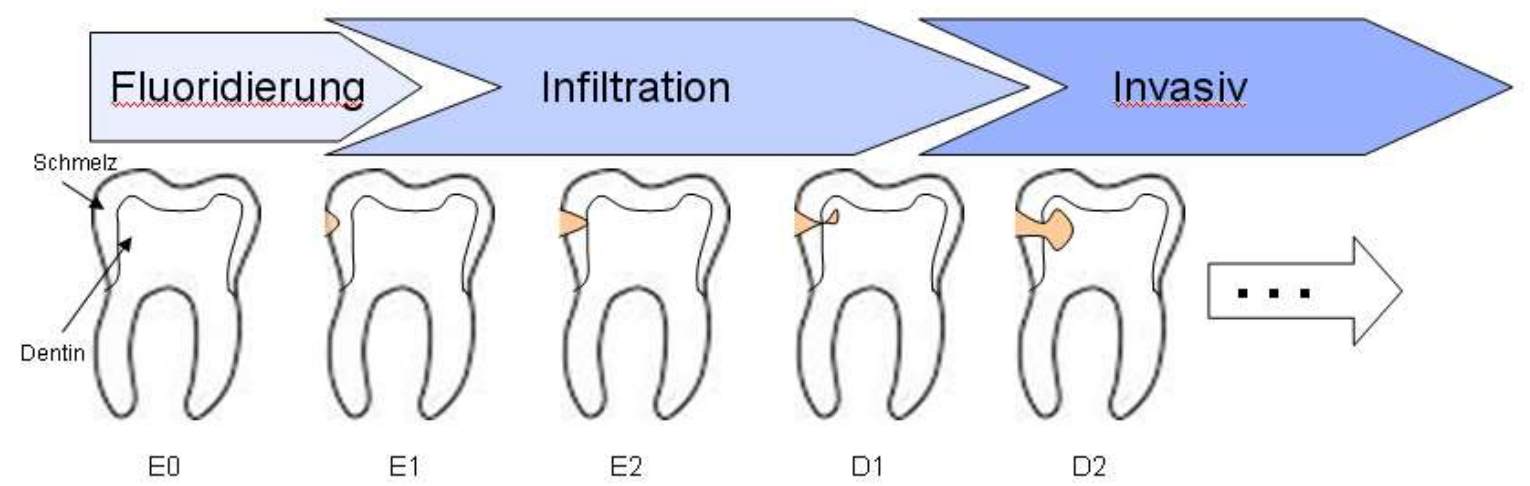

Abbildung 1b: Die Abbildung zeigt die histologische Einteilung der Approximalkaries. Die Einteilung $E$ bezieht sich auf reine Schmelzkaries ohne Dentinbeteiligung. E1- Läsion betrifft die äußere Schmelzhälfte, während die Karies bei E2 bis in die innere Schmelzhälfte reicht. Ab D1 ist das äußere Dentindrittel mitbeteiligt. (D2 bis Dentinmitte) In der Untergruppe D3 (hier nicht abgebildet) ist die Karies bereits in Pulpanähe bis in das innere Dentindrittel extendiert.

Vom Hersteller des Infiltrationskunststoffs Icon (DMG Chemisch-Pharmazeutische Fabrik $\mathrm{GmbH}, 22547$ Hamburg, Deutschland) wird die Kariesinfiltration der Stadien E1 bis D1 empfohlen. Geht die Karies über das erste Dentindrittel hinaus (ab D2), ist eine invasive Behandlung erforderlich.

\subsubsection{Stellenwert von Streptococcus mutans bei der Karies- entstehung}

Es wurde schon vielfach belegt, dass eine korrelierende Prävalenz zwischen Kariesanstieg und dem Auftreten von Mutans-Streptokokken besteht, was bestätigt, dass s. mutans eine entscheidende Rolle bei der Initiation und Progression kariöser Läsionen spielt. Aus diesem Grund wird er auch als Kariesleitkeim bezeichnet (Orland et al. 1954, Orland et al. 1955, Fitzgerald und Keyes 1960, Keyes und Fitzgerald 1962, Krasse 1966, Chang et al. 1997). Diesen besonderen Stellenwert nimmt er vor allem durch seine Stoffwechselleistungen und eine Reihe von Virulenzfaktoren ein (Emilson und Krasse 1985). Mutans-Streptokokken sind in der Lage, enzymatisch Saccharose zu spalten, wodurch extrazelluläre Polysaccharide synthetisiert werden, die eine feste Verbindung zur Zahnoberfläche und somit die Akkumulation einer kariogenen Plaque ermöglichen. Mutans-Streptokokken besitzen außerdem die Fähigkeit, aus aufgenommenen Kohlenhydraten intrazelluläre Polysaccharide als Reservoir bei Substratmangel zu synthetisieren. 
Durch die anaerobe Glykolyse, die die Streptokokken zur Energiegewinnung nutzen, verstoffwechseln sie aufgenommene Kohlenhydrate zu organischen Säuren. Diese werden ausgeschleust und können so zur Entmineralisierung des Schmelzes führen (Hellwig et al. 2009). Außerdem sind Mutans-Streptokokken außerordentlich säuretolerant. So können sie auch bei länger bestehendem niedrigen $\mathrm{pH}$-Wert metabolisch aktiv bleiben. Der intrazelluläre pH-Wert kann von ihnen durch vermehrte glykolytische Aktivität, durch Bildung von Stressreaktionsproteinen, durch Verschiebung des $\mathrm{pH}$ - Optimums für Glukosetransport, Glykolyse und Undurchlässigkeit von Protonen, durch Übergang zum homofermentativen Stoffwechsel, durch gesteigerte Aktivität der $\mathrm{H}^{+} /$ATP-Synthase sowie durch Ausschleusung von Protonen und sauren Endprodukten stabil gehalten werden (Marsh und Martin 2003).

\subsubsection{Prophylaxe}

Bei der Kariesprophylaxe unterscheidet man eine Primär-, Sekundär- und Tertiärprävention. Die Primärprävention zielt auf die direkte Vermeidung der Kariesentstehung, die Sekundärprophylaxe auf ein frühzeitiges Erkennen einer Karies. Die Tertiärprophylaxe beschäftigt sich mit der Behandlung der Erkrankung und dadurch mit der Vermeidung des Progress (Hellwig et al. 2009). Zur Verhinderung einer Karies gelten verschiedene Aspekte als elementar:

Es ist unerlässlich, eine optimale Mundhygiene sicherzustellen (Kneist et al. 2008) und diese durch Remotivation und Reinstruktion des Patienten in regelmäßigen Kontrollterminen während der gesamten kieferorthopädischen Behandlung zu unterstützen (Travess et al. 2004). Dabei sollte der Patient auch über den Einfluss seines Ernährungsverhaltens im Hinblick auf Kariogenität aufgeklärt, dieses mit inm kritisch beurteilt und, falls nötig, umgestellt werden. Mikroorganismen nutzen vor allem niedermolekulare Kohlenhydrate zur Energiegewinnung. Das bedeutet, dass zuckerhaltige Nahrungsmittel die Kariesentstehung begünstigen. In verschiedenen Studien konnte belegt werden, dass ohne bakteriell abbaubare Kohlenhydrate und ohne dass die Nahrung mit plaquebehafteten Zähnen interagiert, keine Karies entsteht (Hellwig et al. 2009). Eine effektive Präventionsstrategie besteht in der zusätzlichen Anwendung von Produkten mit kariesprophylaktischer Wirksamkeit (Kneist et al. 2008, Bergstrand und Twetman 2011). Einer der wichtigsten Grundpfeiler der Kariesprophylaxe sind Fluoride. Es gibt zwei Möglichkeiten, Fluoride zur Prävention von Karies einzusetzen. Zum einen lassen sich Fluoride präeruptiv systemisch zuführen, zum Beispiel in Form von Tabletten oder durch die Nutzung von fluoridiertem Speisesalz. In den USA und der Schweiz wird Trinkwasserfluoridierung als weitere Methode der Kariesprophylaxe genutzt. Diese Möglichkeit wird in Deutschland jedoch nicht 
angewandt, da hier die Beigabe von Zusätzen zum Trinkwasser als Zwangsmedikation eingestuft wurde (Bruker und Ziegelbecker 2000).

Durch die präeruptive systemische Fluoridgabe lagert sich Fluorid während der Zahnentwicklung in die Zahnhartsubstanz ein (Hellwig et al. 2009). Posteruptiv sind lokal applizierte Fluoride für die Kariesprophylaxe noch viel entscheidender, da Fluoride vorwiegend durch Kontakt mit Zahnhartsubstanzen karieshemmend wirken (ten Cate und Featherstone 1991, Hotz 1996). Aufgrund dessen sollten lokale Maßnahmen bevorzugt werden (DGZMK 2000). Dabei arbeitet man mit lokaler Fluoridierung durch Zahnpasta, Spüllösungen, Gele oder hochprozentigere Fluoridlösungen und -lacke die vom Zahnarzt aufgetragen werden. Die günstigsten Fluoridverbindungen sind Natrium-, Amin-, Zinnfluorid und Natriummonofluorphosphat. Fluoride verhindern die Demineralisation, fördern die Remineralisation und vermindern die Säurelöslichkeit des Schmelzes. (Hellwig et al. 2009) Durch den festen Einbau von Fluoriden in das Kristallgitter der Zahnhartsubstanz, die Bildung von Fluorapatit, wird die Kristallinität verbessert, was in geringem Ausmaß auch die Säurelöslichkeit des Schmelzes vermindert (ten Cate und Duijsters 1983).

Die Hauptwirkung der Fluoride ist die Beeinflussung der De- und Remineralisation. Zum größten Teil werden diese Mechanismen von den gelösten Fluoriden, die zudem eine Kalziumfluoridschicht auf der Schmelzoberfläche bilden, in der Umgebung des Zahnes ausgelöst (Fischer et al. 1995). Bei Abfall des pH-Wertes schützt das Fluorid, das in der flüssigen Phase zwischen den Kristallen liegt, den Zahnschmelz indem es von den Hydroxylapatit-Kristallen absorbiert wird und innen so eine fluoridähnliche Eigenschaft verleiht (Hellwig 1996).

Fluoridapatit fällt in Gegenwart von Fluorid hinsichtlich seiner geringen Löslichkeit schneller aus als Hydroxylapatit. Schon bei niedrigem pH-Wert kann die Demineralisationswirkung auf den Schmelz bei beginnender Neutralisation reduziert werden. Folglich werden die Demineralisationsperioden verkürzt, da Mineralien früher beginnen zu präzipitieren. Zusätzlich wird die Widerstandsfähigkeit des Zahns gegenüber weiteren kariösen Angriffen durch den vermehrten Fluoridapatitanteil an der Zahnoberfläche erhöht (Fischer et al. 1995, Hellwig 1996). Zudem hemmen Fluoride zugleich den mikrobiellen Kohlenhydratstoffwechsel, indem die Säurebildung verringert (Marsh 1995) und die Polysaccharidsynthese eingeschränkt wird. 


\subsubsection{White-Spot-Läsionen nach orthodontischer Behandlung}

Für die heutige kieferorthopädische Behandlung ist der Einsatz von festsitzenden Apparaturen, auch Multiband- oder Multibracketapparatur (MB) genannt, unumgänglich und notwendig, weil nicht alle Zahnbewegungen mit herausnehmbaren Geräten realisiert werden können. Diese Therapieform hat jedoch den Nachteil der erschwerten Hygienefähigkeit der Zähne, die über die eingangs beschriebenen Mechanismen eine Entmineralisierung der unzureichend gepflegten Schmelzareale begünstigen.

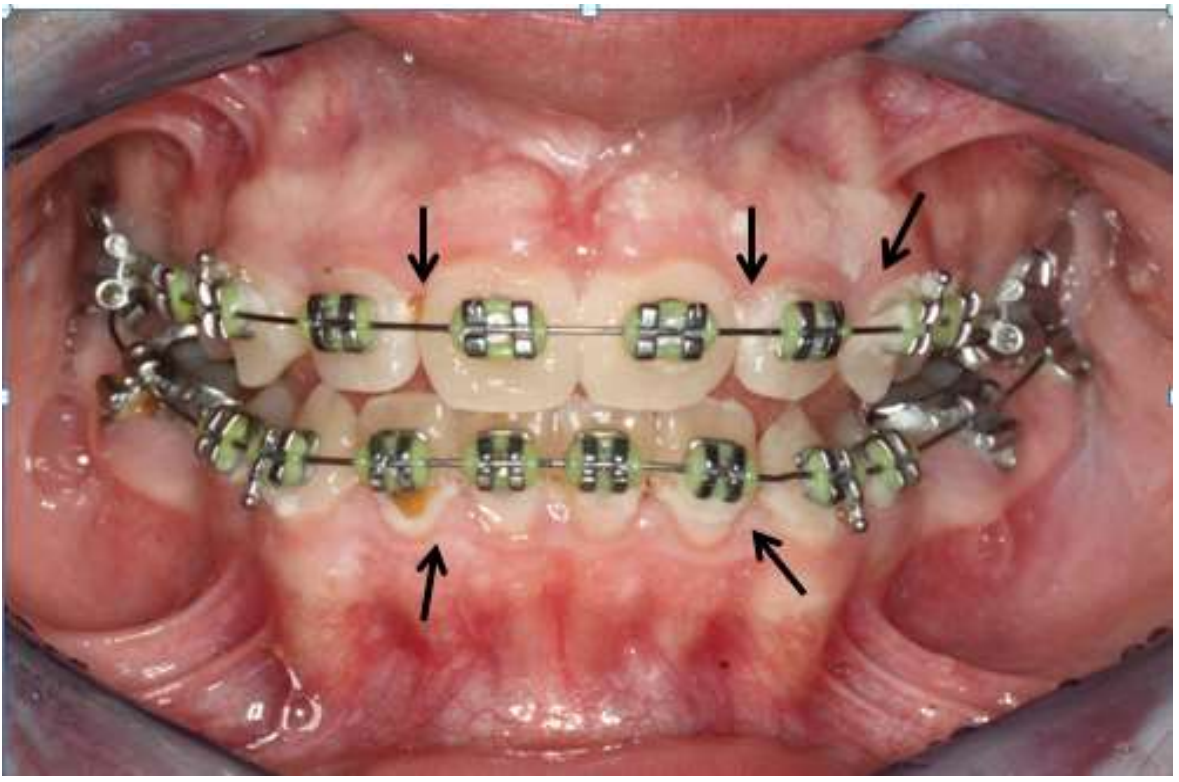

\section{Abbildung 2:}

Die Hygienefähigkeit mit $M B$ ist deutlich erschwert. Vor allem die Bereiche zwischen den Brackets und zwischen Bracket und Zahnfleisch bereiteten vielen Patienten Probleme.

WSL entstehen vor allem während der ersten sechs Behandlungsmonate (Tufekci et al. 2011) und verbleiben als gut sichtbare Läsionen nach Abschluss der festsitzenden Behandlung (Artun und Brobakken 1986, O'Reilly und Featherstone 1987). Dieses Phänomen ist ein besonderes klinisches Problem, seit die direkt auf den Zähnen befestigten kieferorthopädischen Brackets und Bänder eingeführt wurden (Artun und Brobakken 1986). Durch Brackets, Bänder und Bögen ist eine gründliche Zahnreinigung erschwert. Die Zähne sind einer erhöhten Gefahr von Entmineralisierung des Schmelzes ausgesetzt und die Patientenkooperation, die eine optimale Mundhygiene und den Einsatz von Fluoridpräparaten voraussetzt, ist altersbedingt leider oftmals inadäquat (Geiger et al. 1988, Geiger et al. 1992, Shannon 1981).

Nach dem Einsetzen der festsitzenden kieferorthopädischen Apparatur erfolgt häufig eine Verschiebung der Bakterienflora in der Mundhöhle bei der die Anzahl der MutansStreptokokken und Laktobazillen ansteigt und der pH-Wert abfällt, wodurch die Plaque ein kariogenes Potential entwickelt (Balenseifen und Madonia 1970, Lundstrom und Krasse 1987). Diese Erkenntnisse verdeutlichen, warum zusätzlich unzureichende Compliance des 
Patienten einen Risikofaktor darstellt (Geiger et al. 1992, Hadler-Olsen et al. 2011). Festsitzende Apparaturen machen nicht nur die Mundhygiene schwieriger, sondern erhöhen auch die Anzahl der Plaque-Retentionsflächen auf der Oberfläche der Zähne. Kieferorthopädische Patienten entwickeln signifikant mehr WSL als nicht kieferorthopädisch behandelte Personen. Wenn man diese WSL nicht behandelt, kann es im Verlauf zu kariösen Läsionen und zu anhaltenden ästhetischen Problemen kommen (Bishara und Ostby 2008). Demineralisationen treten bevorzugt an den Zähnen des Zahnbogens auf, die einer hohen Aussetzung von Kohlenhydraten Widerstand leisten müssen und gleichzeitig durch eine verringerte Speichelwirkung charakterisiert sind. So konnte während kieferorthopädischen Behandlungen beobachtet werden, dass die Stelle mit der höchsten Inzidenz für Demineralisationen die Frontzähne des Oberkiefers sind. Der Grund für diese Prädelektionsstelle ist eine verminderte Benetzung mit Speichel im Vergleich zu lingualen Schmelzbereichen. Dieser Effekt wird auch durch Patienten mit ausgedehnten WSL an den Labialflächen bestätigt. Entsprechend finden sich weniger Entkalkungen an den dem Speichel direkt ausgesetzten lingualen Flächen (Gorelick et al. 1982). Eine Schmelzdemineralisierung während einer Multibracketbehandlung kann ein extrem schneller Prozess sein, der innerhalb weniger Wochen initiiert wird (Øgaard et al. 1988). Diese Beobachtung wurde von Melrose et al. bestätigt, deren Untersuchung die Möglichkeit der Formation von White-Spot-Läsionen unter klinischen Konditionen in situ innerhalb von nur 4 Wochen zeigt (Melrose et al. 1996). Es handelt sich also um einen sehr schnell fortschreitenden Prozess mit einem besonders starken Anstieg der WSL-Anzahl während der ersten sechs Monate der MB-Behandlung (Tufekci et al. 2011).

Ein Beschleunigen der WSL-Formation kann dabei auch iatrogen erfolgen. In einer aktuellen In-vitro-Untersuchung konnte gezeigt werden, dass Schmelzbereiche, die bei der Bracketbefestigung mit Phosphorsäure angeätzt, aber im Folgenden weder durch Bracketbasis oder Versiegeler abgedeckt werden, eine nochmals signifikant erhöhte Empfänglichkeit für WSL aufweisen. Dieser Faktor ist als besonders kritisch einzustufen bei Vorliegen der Ko-Faktoren einer schlechten Mundhygiene und Anrauzeiten >15 Sekunden (Knösel et al. 2012b). Es liegt damit auch im Verantwortungsbereich des behandelnden Kieferorthopäden, die Problematik der WSL-Formation nicht zusätzlich iatrogen zu verschlechtern.

Nahezu die Hälfte aller festsitzend behandelten Patienten (46\%) entwickelt einer aktuellen Studie zufolge mindestens eine WSL im ersten Behandlungsjahr (Tufekci et al. 2011), während andere Autoren bei sogar $72.9 \%$ der Patienten ein Auftreten von mindestens einer neuen WSL beschreiben. Bei 2.3\% dieser Läsionen sind die Schmelzoberflächen kariös, im Sinne einer Kavitation, eingebrochen (Richter et al. 2011). 


\subsubsection{Therapie}

\subsubsection{Remineralisation bestehender WSL}

Die Demineralisationen, die nach Beendigung der orthodontischen Behandlung zurückbleiben, beeinflussen das ästhetische Endergebnis und sind deshalb von Bedeutung. In einem In-situ-Model zeigten Benson et al. eine Reduktion der physiologischen Remineralisierungsvorgänge bei Anwesenheit von kieferorthopädischen Brackets im Vergleich zu bracketlosen Zähnen (Benson et al. 1999). In manchen Fällen scheinen die Flecken innerhalb des ersten Jahres nach der Entbänderung zurückzugehen (Al-Khateeb et al. 1998, Øgaard B und Ten Bosch 1994, Willmot 2004). Es kann angenommen werden, dass sie abschwächen indem sie über die Jahre weggebürstet werden (Fejerskov et al. 2003). Das Verschwinden könnte auch der Attrition zugeschrieben werden, durch funktionelle Beanspruchung und Zähneputzen (Holmen et al. 1987). Hierzu beschrieben Artun und Thylstrup erstmalig, dass Flächenabrasion durch Auswaschung von Mineralien und Verlust von porösem Gewebe zur Rückbildung von WSL nach Entbänderung führt (Artun und Thylstrup 1986). Aufgrund der Ergebnisse der Studie von Backer Dirks kann davon ausgegangen werden, dass Remineralisierungsprozesse für das Verschwinden von WSL verantwortlich sind (Backer Dirks 1966). Viele Autoren haben den präventiven Effekt von Fluoriden auf die Ausbildung von WSL (Tezel et al. 2002, Øgaard et al. 2001, Kleber et al. 1999) und die Remineralisation von WSL aufgezeigt. Dazu zeigten Donly et al. in vitro, dass beim Zementieren von Bändern Fluoride-freisetzende Glasionomerzemente die Remineralisation von WSL unterstützen (Donly et al. 1995). Geiger et al. haben festgestellt, dass das Auftreten von WSL merklich reduziert werden könnte, würde man täglich eine Natruimfluoridlösung nutzen (Geiger et al. 1988). Dubroc et al. zeigten experimentell, dass fluoridfreisetzende Produkte die Demineralisierungen um Brackets herum reduzieren (Dubroc et al. 1994). Laut Demito et al. ist die mittlere Tiefe von Schmelzdemineralisierungen nach der Applikation von Fluoridlacken, im Gegensatz zu der Kontrollgruppe, ohne Fluorudierung bis zu 38\% geringer (Demito et al. 2004). Kalha und Benson et al. empfahlen, dass Patienten mit festsitzender Zahnspange täglich mit Natriumfluoridlösung spülen sollten, da dies den Schweregrad der WSL reduziere (Kalha 2004, Benson et al. 2004). Folglich ist zu sagen, dass eine optimale Mundhygiene die Grundvoraussetzung für die Vermeidung von WSL ist. Andere präventive Maßnahmen beinhalten die Applikation und Verwendung von fluoridfreisetzenden Materialien (wie z.B. fluoridfreisetzende Zemenente, Bondings oder Fluoridlacke) und das tägliche Spülen mit fluoridhaltigen Mundspüllösungen (Kalha 2004, Benson et al. 2004, Øgaard et al. 2001). Obwohl gezeigt werden konnte, dass die Applikation 
von Fluoriden die Entstehung von WSL reduzieren kann und bei der Remineralisierung des Schmelzes hilft und obwohl mittlerweile viele präventive Maßnahmen in der Kieferorthopädie angewandt werden, wie zum Beispiel der Einsatz von fluoridfreisetzenden Zementen und regelmäßige Prophylaxe mit Reinigung und anschließender Fluoridierung, bleiben WSL trotzdem bei einer Vielzahl von Patienten ein Problem. Die milchige Farbe der WSL kann das ästhetische Erscheinungsbild negativ beeinträchtigen, was die Zufriedenheit mit der kieferorthopädischen Behandlung und deren Ergebnis schmälert.

\subsubsection{Therapie der White-Spot-Läsionen}

Im Falle von kariösen Läsionen sind Restaurationen unabdingbar. Nicht eingebrochene Läsionen sollten mit dem Therapieziel behandelt werden, sie zu remineralisieren oder zumindest die Progression aufzuhalten (Stahl und Zandona 2007).

Die klassischen Herangehensweisen zur WSL-Therapie lassen sich also in non-invasive (konservierende) und invasive Maßnahmen gliedern. Non-invasive Maßnahmen beinhalten das Remineralisieren des Schmelzes durch Anwendung lokaler Fluoride (Backer Dirks 1966) sowie die begleitende Abrasion durch das Zahnbürsten. Zusätzlich spielt die Aufklärung über kariogene Nahrung eine Rolle. Auch wenn somit Läsionen inaktiviert werden, bleibt der optisch nachteilige Effekt. Die WSL bleiben klinisch sichtbar (Gugnani 2012, Mattousch 2007).

Trotz der Remineralisation ist die komplette Farbangleichung der WSL kaum möglich. Oftmals verfärben sich arretierte Läsionen auch bräunlich, da während der Remineralisierung Farbstoffe eingelagert werden (Hellwig et al. 2009). Das bedeutet, dass das ästhetische Problem nicht nur weiterhin bestehen bleibt, sondern sich sogar verschlechtern kann. Non-invasive Behandlungsmethoden sind zu bevorzugen, wenn sie das Langzeit-Kariesrisiko reduzieren, indem sie äthiologische Faktoren der Karies beeinflussen und den natürlichen Reparaturprozess der Zähne fördern (Pitts 2004). Dabei hängt ein nicht invasives Herangehen allerdings sehr von der guten Compliance eines Patienten ab und ist in fortgeschrittenen Stadien eines kariösen Prozesses ineffektiv (Kidd et al. 2008).

Alternativ zur non-invasiven Behandlung kann eine invasive Therapie erfolgen, bei der das kariogene Gewebe entfernt und wie bei einer Füllung durch Composite-Material ersetzt wird. Dies bedeutet jedoch auch die Entfernung von erheblichen Mengen umgebender gesunder Zahnhartsubstanz. Trotz einer minimalinvasiven Zielsetzung (Vila Verde et al. 2009) müssen für diese Art der restaurativen Therapie immer auch wesentliche Mengen des umliegenden Gewebes entfernt werden, um alle kariösen Stellen zu erreichen und zu entfernen. Zusätzlich fängt für den Zahn, durch den ersten invasiven Eingriff, oft ein Kreislauf von Behandlung und Nachbehandlung an. Restaurationen haben eine limitierte Haltbarkeit und 
werden je nach Qualität und Füllungstyp früher oder später als insuffizient eingestuft und erneuert, was als "death spiral of restaurations" bezeichnet wurde (Qvist 2008). Deshalb sollte der erste invasive Eingriff, solange es möglich und angemessen ist, hinausgezögert werden. Sowohl für nicht invasive als auch für restaurative Behandlungsoptionen besteht jeweils das Risiko der Unter- oder der Überversorgung (Nuttal und Pitts 1990).

Ein vergleichsweise neuer Ansatz, die Progression von White-Spot-Läsionen aufzuhalten, ist die Kariesinfiltration mit einem niedrig viskösen lichthärtenden Kunststoff (Infiltrant), ohne das im Rahmen von invasiven Füllungstherapien übliche rotierende Entfernen von Läsion und angrenzender gesunder Zahnhartsubstanz (Paris et al. 2010).

Kariesinfiltration ist somit eine neuartige minimalinvasive WSL-Therapiemöglichkeit, die die Mittelposition zwischen den non-invasiven und den invasiven WSL-Behandlungsmethoden einnimmt (Paris und Meyer-Lueckel 2010). Ziel ist das Aufschieben oder Verhindern des Beginns der invasiven Füllungstherapie-Spirale.

Klinische Studien zur Kariesinfiltration zeigen, dass die Progression initialer, nicht eingebrochener Läsionen aufgehalten werden kann. Sogar das Fortschreiten von eingebrochenen Läsionen kann durch Versiegeln mit dentalen Adhäsiven verlangsamt werden. (Griffin et al. 2008)

Am Anfang der Idee der Läsions-Infiltration stand die Versiegelung der Karies mit einfachen dentalen Adhäsiven. Im Gegensatz zu dieser konventionellen Kariesversiegelung, bei der eine Kunststoffschicht auf die Schmelzoberfläche gelegt wird, hat die Kariesinfiltration das Ziel, den porösen Läsionskörper mit einem niedrig-viskösen Kunststoff zu tränken und anschließend ausgehärtet zu werden, wodurch die Diffusionswege für kariogene Säuren blockiert und die Läsionen verschlossen werden (Paris et al. 2007a und b). Dadurch wird die Progression der Karies verlangsamt oder sogar unterbunden. Die Fähigkeit des Kunststoffs, in diese Läsionsporositäten zu penetrieren, wurde schon vor 30 Jahren beschrieben (Davila et al. 1975). Seitdem wurde die Penetration und das Inhibieren künstlicher Läsionen durch Adhäsive und Fissurenversiegler in verschiedenen Laborstudien untersucht (Rodda 1983). Jedoch sind herkömmliche Versiegler und Adhäsive nicht für hohe Penetrationsfähigkeit hergestellt und erreichen deshalb nur eine geringe Durchdringtiefe in die natürlichen Schmelzläsionen (Paris et al. 2007b). Spezielle Kunststoffe, deren Viskosität für schnelle Kapillarpenetration optimiert wurden, penetrieren bzw. infiltrieren den erkrankten Schmelz signifikant tiefer und werden daher als Infiltrant bezeichnet (Meyer-Lueckel und Paris 2008a und b). Die Resultate bisheriger Studien zeigen, dass die Kariesinfiltration eine klinisch anwendbare Methode ist, um die Progression der Karies signifikant zu reduzieren (Paris und Meyer-Lueckel 2010). 
Um jedoch die Farbe der WSL an die Zähne anzugleichen, gab es bisher keine etablierte Therapie. Eine Möglichkeit inaktive WSL zu behandeln wurde 2007 von Knösel et al. vorgestellt (Knösel et al. 2007). Dabei handelt es sich um eine Bleachingtherapie des gesamten Zahnes, bei dem sich die Farbe des umliegenden Zahnschmelzes der des WSL angleicht. Der hauptsächliche Nachteil hierbei ist allerdings die Dauerhaftigkeit. Das Ergebnis rezidiviert nach anfänglich guten Ergebnissen nach ca. sechs Monaten ebenso schnell wie bei einem herkömmlichen Zahnbleaching (Knösel et al. 2011). Auch Atrittion führt zu einem gewissen Teil zum Farbangleich der WSL an die AGS (Holmen et al. 1987, Artun und Thylstrup 1986). Diese Möglichkeit lässt sich jedoch nicht sinnvoll als Therapiemittel einsetzen. Eine besondere Charakteristik des Infiltrationskunsstoffes (Icon, DMG ChemischPharmazeutische Fabrik GmbH, 22547 Hamburg, Deutschland) ist sein spezifischer Lichtbrechungsindex: Dieser ist dem des gesunden Schmelzes sehr ähnlich (Abbildung 3) (Paris und Meyer-Lueckel 2009). Daraus ergibt sich ein positiver Nebeneffekt der Kariesinfiltration: Die Farbe der WSL gleicht sich an die benachbarten angrenzenden Schmelzbereiche an, wodurch eine kariesprotektive Behandlung mit einer ästhetischen Korrektur verbunden werden kann. Fallstudien und In-vitro-Studien zeigen eine reduzierte Sichtbarkeit der infiltrierten WSL (Neuhaus et al. 2010, Rocha et al. 2011).

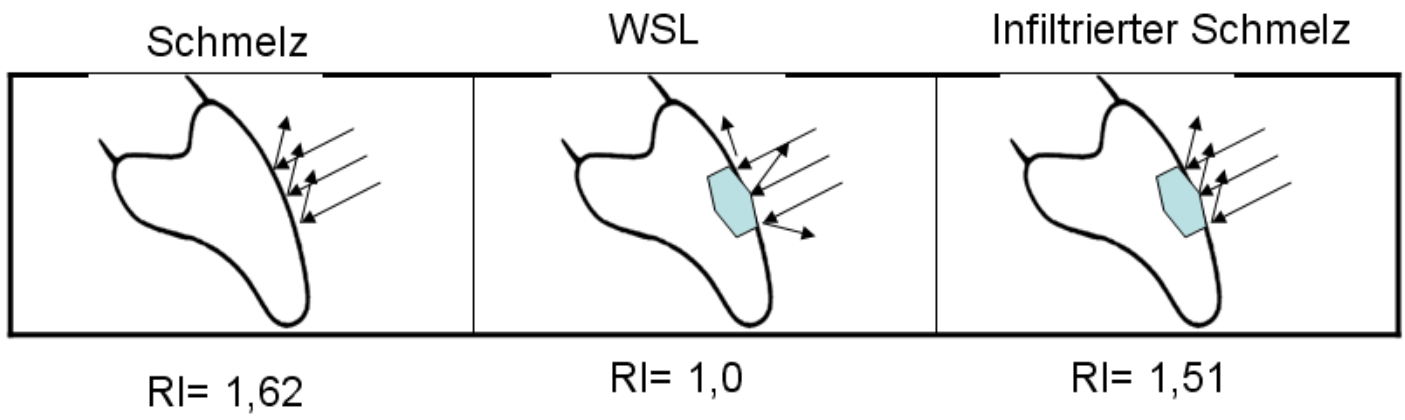

Abbildung 3: Während gesunder Schmelz einen Lichtbrechungsindex (Refractive Index, RI) von 1,62 aufweist, liegt der RI von WSL bei nur 1: Einfallendes Licht wird gestreut statt reflektiert, weswegen WSL opak erscheinen. Der RI des Infiltrationskunststoffs Icon ähnelt mit einem Wert von 1,51 sehr dem des gesunden Schmelzes, weshalb infiltrierte WSL und angrenzender gesunder Schmelz farblich schlecht zu unterscheiden sind (Paris und MeyerLueckel 2009). 


\section{Ziele der Arbeit}

Der Infiltrationskunststoff Icon (DMG, Hamburg, Deutschland) bewirkt laut In-vitro-Studien an bovinen (Mueller et al. 2006) und humanen Zähnen (Paris et al. 2007b) sowie nach Kasuistiken neben der kariespräventiven Wirkung zusätzlich auch ein ästhetisches Kaschieren der weißen bis bräunlichen White-Spot-Läsionen. Dieser Camouflage-Effekt erklärt sich durch die vergleichbaren Lichtbrechungs-Indices von gesundem Schmelz und dem Infiltrationskunststoff (siehe Abbildung 3, Seite 16). Da Informationen über die Langzeitbeständigkeit dieses Camouflage-Effekts während der In-vivo-Alterung des Infiltranten in der Mundhöhle nicht vorliegen, sollten dessen Ausmaß und Dauerhaftigkeit durch eine objektive, spektrophotometrische Farb- und Helligkeits-Analyse (CIE-L*a* $\mathrm{b}^{*}$ Analyse) zum ersten Mal in vivo langzeituntersucht werden.

Primäres Ziel der Arbeit war das Messen der Farb- und Helligkeitsunterschiede (CIE-L*a*b*$\triangle E-W e r t e)$ von multibracketinduzierten WSL und AGS in vivo vor (Baseline, t0) und nach Infiltration (t1) sowie über sechs Monate nach Infiltration, (t2, Woche 1; t3 Monat 1; t4, Monat 3; t5, Monat 6 nach Infiltration) im Vergleich zu unbehandelten Kontrollzähnen, um das Ausmaß der Angleichung und die Haltbarkeit des ästhetischen Effekts zu bewerten.

Es wurde die Nullhypothese getestet, dass Farb- und Helligkeitsunterschiede zwischen WSL und AGS 6 Monate nach Infiltration (t5) nicht signifikant verschieden von den Unterschieden der äquivalenten Areale auf unbehandelten Kontrollzähnen sind (delta-E Werte WSL/ AGS t0 vs. delta-E Werte WSL/ AGS t5).

Da als Teil der mit den Patienten getroffenen Vereinbarung die Kontrollzähne nach 6 Monaten ebenfalls infiltriert wurden, entfallen die Kontrollzähne nach Zeitpunkt t5.

Das sekundäre Ziel der Arbeit war das Nachuntersuchen der Farb- und Helligkeitscharakteristika der zu t0 infiltrierten Flächen über weitere sechs Monate (t6, Monat 12 nach Infiltration) im Vergleich zur Baseline (t0). 


\section{Material und Methode}

\subsection{Der Infiltrant (Icon)}

Das in dieser Studie zur Behandlung der WSL verwendete Produkt Icon ist ein an der Universitätszahnklinik der Charité (Berlin) entwickeltes, zugelassenes und seit 03/2009 kommerziell erhältliches dentales Füllungsmaterial (Icon, DMG Chemisch-Pharmazeutische Fabrik GmbH, 22547 Hamburg, Deutschland). Aufgrund seiner sehr niedrigen Viskosität ist dieser Werkstoff zum Infiltrieren von demineralisierten Läsionen geeignet und wird als Infiltrant bezeichnet.

Zum Lieferumfang eines Icon-Kits gehören 0,30 ml Icon-Etch (in Spritzenform mit Vestibular-Tips; Inhaltsstoffe: 15\%iges Salzsäuregel, pyrogene Kieselsäure, oberflächenaktive Substanzen) zum Konditionieren des demineralisierten Schmelzes, um die harte, pseudointakte Schmelzoberfläche der WSL zu reduzieren. Hierdurch wird, dem niedrigviskösen Icon-Kunststoff die Penetration in tiefere, demineralisierte Schmelzschichten ermöglicht. Des Weiteren gehört eine Trocknungsflüssigkeit (Icon-Dry, 0,45 ml; in Spritzenform mit Luer-Lock-Tip; Inhaltsstoff: 99\% Ethanol) für die absolute Trockenlegung der Läsion zum Lieferumfang. Icon-Dry ist ein hochreines Ethanol, das verwendet wird um die Läsion bis in die Tiefe ausgiebig zu trocknen. Da Feuchtigkeitsrückstände in den Poren eine Infiltration verhindern würden, ist der Trocknungsschritt sehr wichtig. Der Icon-Infiltrant liegt ebenfalls in Spritzenform à $0,45 \mathrm{ml}$ vor (Inhaltsstoffe: Methacrylat-basierte Harzmatrix, Initiatoren, Additive). Durch Kapillarkräfte wird die schnelle Penetration des Infiltranten in die freigelegten Schmelzporen ermöglicht. Der niedrigvisköse, Kunststoff härtet unter Lichteinwirkung aus. Bedingt durch die Enge der Kapillaren in der Läsion ist eine Einwirk-und Penetrationszeit von drei Minuten erforderlich, um die vollständige Infiltrierung zu gewährleisten (Meyer-Lueckel et al. 2007a und b).

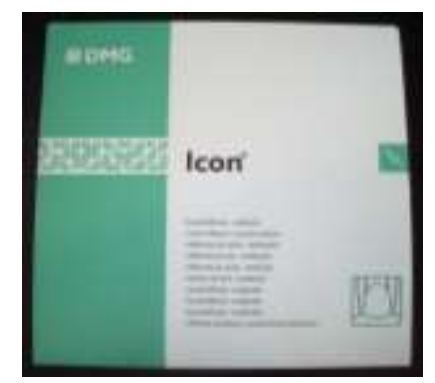

Abbildung 4a

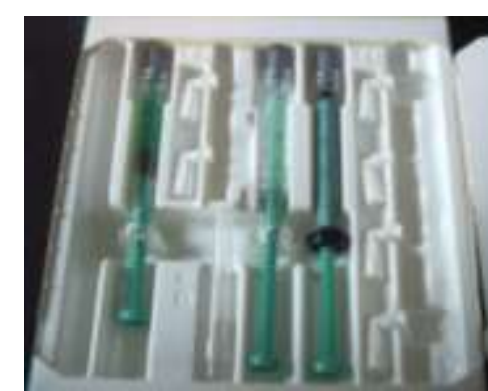

Abbildung 4b

Abbildungen $4 a$ und $b$ zeigen das Icon-Patienten-Kit (4a) und dessen Inhalt (4b). 


\subsection{Dentale Farb- und Helligkeitsbestimmung}

\subsubsection{Ablauf der Studie}

In der vorliegenden Studie wurde das Ausmaß der Farbangleichung der WSL an den umliegenden Schmelz und die Haltbarkeit dieses Effekts bestimmt, indem die CIE-L*a*b*Daten vor der Infiltration (Baseline, t0), direkt nach der Infiltration (t1), nach einer Woche (t2), vier Wochen ( $\mathrm{t} 3$ ), drei Monaten ( $\mathrm{t} 4)$ und nach sechs Monaten ( $\mathrm{t} 5$ ) verglichen wurden.

Die CIE-L*a*b*-Farbbestimmung erfolgte mit einem der derzeit am bestmöglich reproduzierbar messenden, kommerziell erhältlichen Spektrophotometer (ShadePilot, Degudent, Hanau-Wolfgang, Deutschland, Abbildung 5a) (Karamouzos et al. 2007)). Mit dem Spektrophotometer-Handmessgerät werden non-invasiv und somit reproduzierbar und unter standardisierten Bedingungen CIE-L*a*b*- Farb- und Helligkeitswerte ermittelt. Der Unterschied zum Gros der erhältlichen Zahnfarbbestimmungsmethoden besteht in der Durchführung der spektrophotometrischen Messung bei geschlossenen Lippen und damit ohne den verzerrenden Einfluss des Umgebungslichtes. Durch eine Speicherung des gemessenen Areals für weitere Messungen wird der zweite häufigste Fehler der variierenden Kopfposition auf die gemessene Zahnfarbe ausgeschlossen (Knösel et al. 2009) (Abbildung $5 b)$. Eine Kontrolle erfolgt über eine Digitalkamera, die mit einem extraoral ablesbaren Bildschirm verbunden ist. Das Gerät verzeichnet über einen Farb- und Helligkeitssensor die Luminanz und die Chrominanz der Schmelzfläche als Wellenlänge des Lichtes. Die erfassten Daten wurden auf einem Computer gespeichert und mit dem dazugehörigen Programm (Degudent Shadepilot, Hanau-Wolfgang, Deutschland) ausgewertet. Dabei wurden die WSL und die AGS markiert und die jeweiligen CIE-L*a* $\mathrm{b}^{*}$-Werte für die spätere statistische Auswertung bestimmt (Abbildung 5c). 


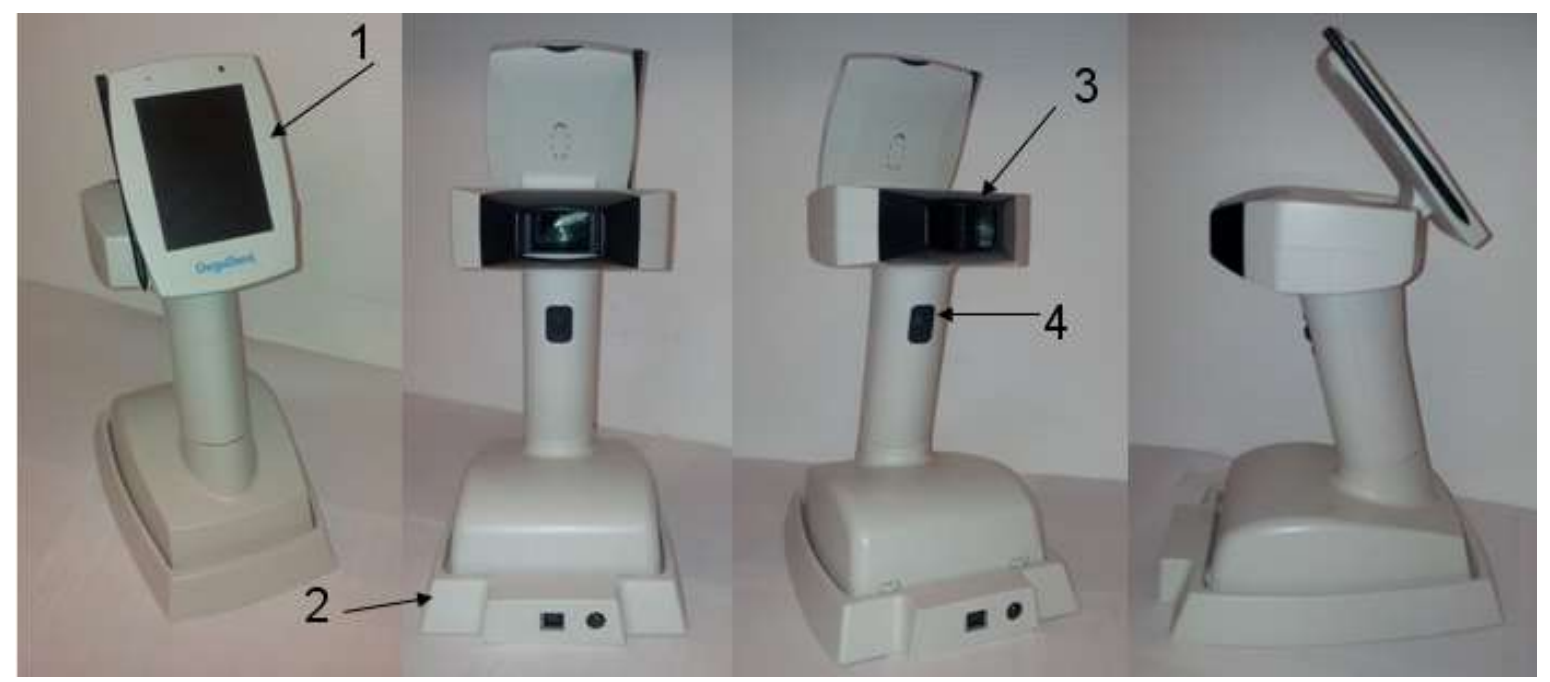

\section{Abbildung 5a:}

Das Spektrophotometer-Handmessgerät (ShadePilot, Degudent, Hanau-Wolfgang, Deutschland), das zur Farb- und Helligkeitsmessung verwendet wurde. (1 Bildschirm, 2 Ladestation und Verbindungsstation zum Computer, 3 Kamera, 4 Auslöseknopf)

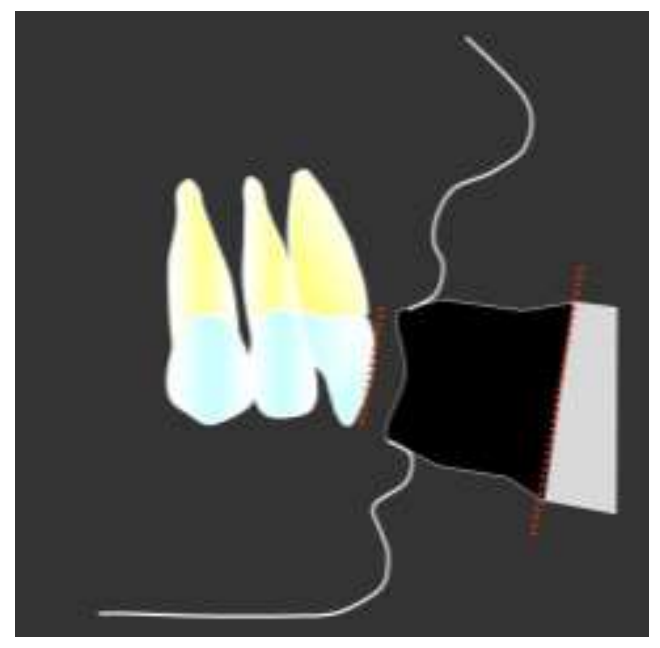

\section{Abbildung 5b:}

Die spektrophotometrische Messung wurde bei geschlossenen Lippen durchgeführt. So kann sichergestellt werden, dass die für eine In-vivo-Studie typischen Fehlerquellen -

Einfluss des Umgebungslichts oder verschiedener Kopfpositionen auf die Zahnfarbe - ausgeschlossen werden können. (Knösel et al. 2009).

(Abbildung nach Knösel et al. 2013 S.89) 


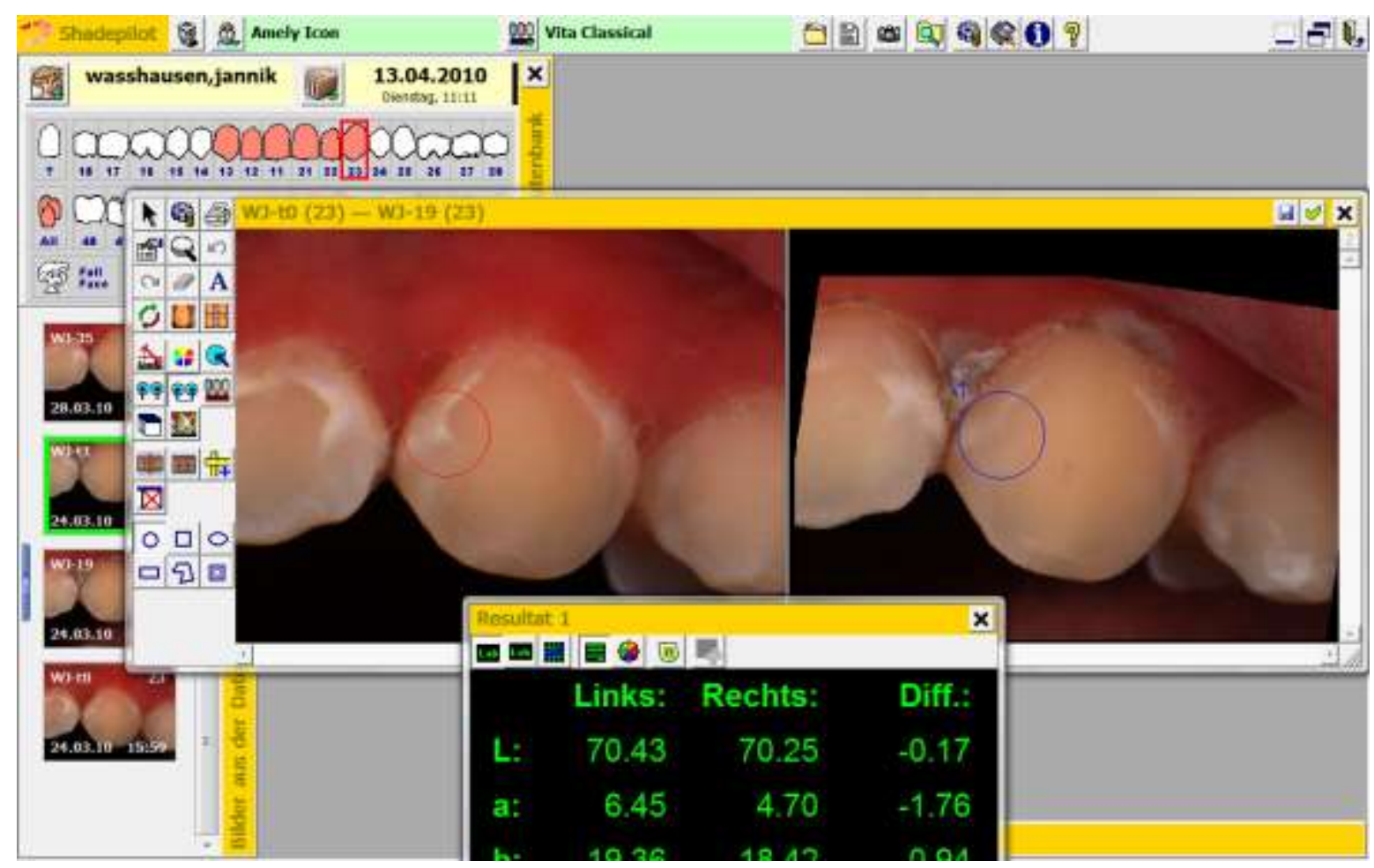

Abbildung 5c: Die Auswertung der von der integrierten Digitalkamera des Spektrophotometers erfassten Bilder und die Bestimmung der festgelegten Bereiche erfolgte mit dem zugehörigen Programm. So wurden die CIE-L*a*b*-Werte für die festgelegten Bereiche bestimmt, die Umrisse der gemessenen Flächen gespeichert und für alle weiteren Follow-up-Messungen genutzt. Auf diese Weise wurde die exakte Re-Lokalisation der Flächen sichergestellt, was besonders im Falle der infiltrierten und damit oft völlig kaschierten Läsionen von großer Bedeutung für die Qualität der Untersuchung war. (Abbildung nach Knösel et al. 2013 S.89) 


\subsubsection{CIE-L*a*b*-Werte}

Die CIE-L* $a^{*} b^{*}$-Farb-Werte geben die Intensität, die Helligkeit und den Farbwert im dreidimensionalen Farbraum wieder. Die $L^{*} a^{*} b^{*}$-Analyse beinhaltet drei Parameter. Der erste Parameter ist der ,Lightness' (L*)-Wert; er beschreibt die Helligkeit des Zahns. Die beiden anderen Parameter sind Farbparameter. Der $a^{*}$-Wert gibt die Position auf der Rot-GrünAchse an, der $b^{*}$-Wert diejenige auf der Gelb-Blau-Achse. Diese Art der Farbdarstellung erlaubt eine exakte Definition jedes Punktes im dreidimensionalen Farbraum und wurde von der Commission International d'Eclairage (CIE) 1976 als internationaler Standard (DIN 6174) zur Farbbestimmung festgelegt (Johnston 2009, Commission Internationale de l'Eclairage 2004).

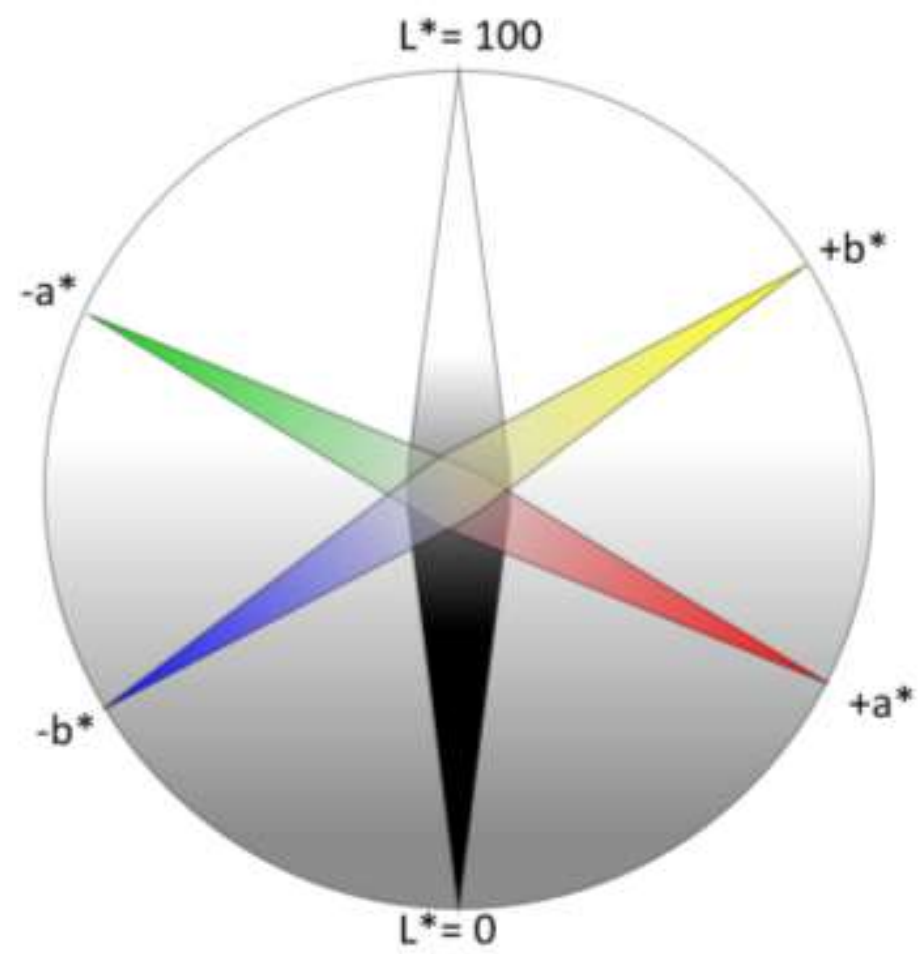

\section{Abbildung 6:}

Dreidimensionale Darstellung des L*a*b*-Systems 


\subsubsection{Dentale Farbbestimmung: Technische Problemstellungen}

Mit der Zahnfarbbestimmung geht die Schwierigkeit einher, die befundete Farbe oder Helligkeit mit anderen zu kommunizieren. Dies führte zur Entwicklung unterschiedlicher Farbtabellen und -skalen. Ein klassisches Beispiel ist der Farbraum nach Munsell, der die Parameter Farbton, Farbwert und Chroma definiert (McLaren 1987).

Die menschliche Farbwahrnehmung beinhaltet das Spektrum des sichtbaren Lichts, das in das Auge eintritt, dort von den drei verschiedenen Farbrezeptoren der Retina (rot, grün blau) verarbeitet wird, die wiederum die Information über den Nervus opticus an das Gehirn weiterleitet (Ishikawa-Nagai et al. 2007, Hecht 2002).

Eine Methode zur Bestimmung der natürlichen Zahnstruktur- und -farbe ist wissenschaftlich nur dann geeignet, wenn eine Reproduzierbarkeit der Messungen gegeben ist.

Bei der dentalen Farbbestimmung ergibt sich in der Praxis die klinische Problemstellung inhomogener und gekrümmter Schmelzoberflächen. Eine Variation des Betrachtungswinkels oder differierende Angulation des Farbmessgerätes zur Schmelzfläche bei Folgemessungen können die Farbwahrnehmung verzerren (Knösel et al. 2009). Durch den variierenden Durchmesser und die Dicke des Schmelzmantels ist zudem die Transluzenz der Zähne eine kompliziert zu erfassende Größe bei der Farbmessung. Die Helligkeit desselben Zahns kann bei geöffnetem Mund trotz gleichbleibendem Betrachtungswinkel anders wahrgenommen werden, wenn z.B. die Zunge während der Messung hinter den Zahn gelegt wird, also in Abhängigkeit des Hintergrunds (Johnston 2009).

Die Helligkeit und Farbe resultiert aus dem Ausmaß der Streuung des Lichts. Helles Licht folgt z.B. höchst unregelmäßigen Lichtpfaden durch den Zahn, bevor es an der Oberfläche entsteht und das Auge des Betrachters erreicht (O'Brien et al. 1990, Van der Burgh et al. 1990). Das ist auch der Grund, weshalb WSL sichtbar sind. Während gesunder Schmelz einen Lichtbrechungsindex (RI) von 1,62 hat, liegt der RI von WSL bei nur 1. Das heißt, die Lichtstreuung ist stark unterschiedlich. Der Infiltrarionskunststoff Icon ähnelt mit seinem RI von 1,51 sehr dem des gesunden Schmelzes (Abbildung 3, Seite 16), weshalb sich die Farbe der WSL nach der Infiltrarion auch der des AGS angleicht (Paris und Meyer-Lueckel 2009) (siehe Abbildung 3 Seite 16).

Die Zahnfarbe ist durch den kombinierten Effekt der intrinsischen und extrinsischen Färbung festgelegt (Watts und Addy 2001). Die intrinsische Zahnfarbe hängt mit der Lichtstreuung und den -absorptionseigenschaften von Schmelz und Dentin zusammen (Ten Bosch und Coops 1995). Die extrinsische Zahnfarbe ist assoziiert mit der Absorption von Tabak- oder 
Lebensmittelbestandteilen wie z.B. nach häufigem Tee-, Kaffee- oder Rotweinkonsum, die sich jedoch durch Schmelzpolituren oder Bleichverfahren gut entfernen lassen (Joiner et al. 1995). Weniger leicht zu verändern sind dagegen intrinsische Verfärbungen, zu denen auch die WSL gehören.

Zur Beurteilung der Zahnfarbe gibt es verschiedene Möglichkeiten. Die älteste Methode ist der visuelle Vergleich mit Farbskalen. Sie findet häufig in der zahnmedizinischen Praxis Anwendung. Hierzu benutzt man Farbringe, die mit einem standardisierten Farbspiegel ausgestattet sind (Hammad 2003, Fani et al. 2007). Der standardisierte Farbspiegel hat eine Einteilung mit Zahnfarben von A bis D (gelblich, rötlich, bläulich und gräulich) und von 1 (hell) bis 4 (dunkel).

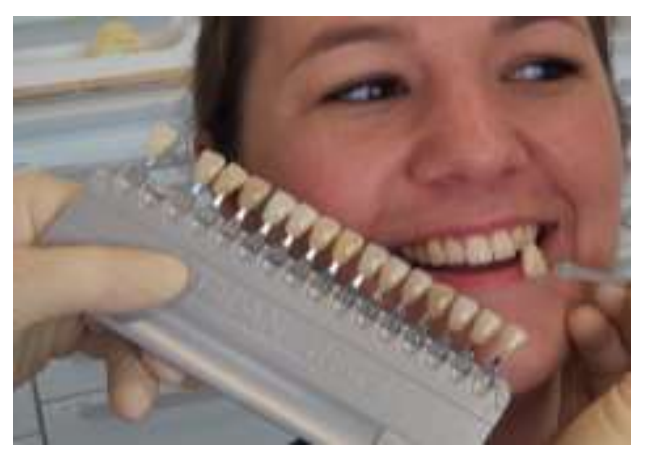

\section{Abbildung 7:}

Die Farbbestimmung mithilfe eines standardisierten

Farbspiegels.

Dieses Verfahren hat allerdings den entscheidenden Nachteil, dass es mit dem menschlichen Auge durchgeführt wird und somit subjektiv ist. Verschiedene Faktoren, wie etwa die Farbe des Umfeldes und die Helligkeit, beeinflussen die Farbempfindung (Backer Dirks 1966, Johnston 2009). Auch die Oberfläche des Zahnes und der Blickwinkel verändern die wahrgenommene Farbe ebenso wie die subjektive psychische und physische Befindlichkeit. Alle diese Einflüsse erschweren die visuelle Farbanalyse mittels Farbringen und ermöglichen nur eine begrenzt zuverlässige und schwer reproduzierbare Datenerhebung (Backer Dirks 1966). Die Reproduzierbarkeit der visuellen Farbbestimmung liegt bei lediglich 46,6\% (Paul et al. 2002); sie ist für wissenschaftliche Zwecke daher nicht geeignet.

Eine objektivere Möglichkeit zur dentalen Farbanalyse bieten elektronische Farbmessgeräte, wie Colorimeter (z.B. Shade Eye, Shofu Dental Corporation, Ratingen, Deutschland) oder Spektrophotometer (z.B. ShadePilot, Degudent, Hanau-Wolfgang, Deutschland, Abbildung 5 a S.20), die eine digitale sowie parametrische und damit reproduzierbare Farbbestimmung auch kleinster Farbdifferenzen ermöglichen (Okubo et al. 1998).

Basierend auf der Grundlage dessen, was die Rezeptoren des menschlichen Auges erfassen können, reduzieren Colorimeter mit Hilfe von Filtersystemen die gemessene Farbinformation, indem nur das Licht der verschiedenen Farbkomponenten gemäß den 
Farbrezeptoren (rot, grün blau) des Auges getrennt verzeichnet wird. Dies entspricht einer vereinfachten Anpassung an die menschliche visuelle Reaktion auf Lichteinfall, beinhaltet jedoch keine weiteren, für das Auge nicht erkennbaren Daten. Zu diesen gehören spektrale Informationen oder Daten, die z.B. mit einem Spektralphotometer zusätzlich gemessen werden können. Ein Spektralphotometer bricht das Licht in ein Spektrum und verwendet dabei z.B. ein Farbgitter. Sensoren lesen jeden Abschnitt des Spektrums aus, registrieren die Intensitätsverteilung und erzeugen spektrale Datensätze, die auch Informationen im infraroten oder ultravioletten Licht beinhalten.

Spektralphotometer sind aufgrund ihrer Genauigkeit vorwiegend für wissenschaftliche Zwecke prädestiniert. Spektrale Datensätze können über Algorithmen beliebig in die gängigen, standardisierten $\mathrm{CIE}-\mathrm{L}^{*} \mathrm{a}^{*} \mathrm{~b}^{*}$-, RGB oder $\mathrm{XYZ}$-Farbsysteme transferiert werden. Dies ermöglicht die Vergleichbarkeit mit den Ergebnissen anderer Forschungsgruppen (Tzeng und Berens 2005, Johnston 2009).

In der Studie von Okubo et al. wird die Reproduzierbarkeit elektronisch-instrumenteller Farbanalysen mit $83,3 \%$ bis $100 \%$ angegeben (Okubo et al. 1998). Elektronische Messverfahren haben in der Mehrheit die Verwendung der CIE- $L^{*} a^{*} b^{*}$-Farbraum-Analyse als ein objektives, standardisiertes System gemeinsam, das sich zum Standard in der zahnmedizinischen Forschung entwickelt hat (Ishiwata 1986, O‘Brien et al.1990b).

Die CIE definiert eine Umwandlung der Tristimulus-Werte in die Werte des CIE- $L^{*} a^{*} b^{*}$ Farbraum auf drei räumlich angeordneten Achsen $L^{*}, a^{*}$ und $b^{*}$ (Commission Internationale de l'Eclairage (CIE) 2004) (siehe Abbildung 6, Seite 22). Es handelt sich um einen einheitlichen Farbraum, bei dem gleich wahrgenommene Farbunterschiede mit gleichen Abständen entsprechend korrespondieren. Der $L^{*}$-Wert ist ein Maß, das die Helligkeit eines Objekts beschreibt. Er ist auf der Skala so eingeteilt, dass vollständiges ,Schwarz einen $L^{*}$ Wert von null und ein komplett abspiegelnder Diffusor (,Weiß') einen $L^{*}$-Wert von 100 erhält. Der positive $a^{*}$-Wert ist das Maß für den Farbton ,Rot' ohne andersfarbige Einflüsse, der negative $a^{*}$-Wert entsprechend der Farbe ,Grün'. Der $b^{*}$-Wert steht im Positiven für den Farbton ,Gelb' und im Negativen für ,Blau'. Die $a^{*}$ - und $b^{*}$-Koordinaten, die sich Null annähern, bedeuten neutrale Farben wie ,Weiß' oder ,Grau'. Der entscheidende Vorteil des CIE-L*a*b*-Systems besteht also darin, dass Farbunterschiede zu Einheiten parametrisiert werden, die in Relation zur visuellen Erfassung gesetzt werden und statistisch ausgewertet werden können. 
Der CIE-L*a*b*-Farbraum ermöglicht das einfache Berechnen von Farbunterschieden zwischen zwei Flächen:

$\Delta E_{\mathrm{p}, \mathrm{v}}=\sqrt{\left(L_{\mathrm{p}}^{*}-L_{\mathrm{v}}^{*}\right)^{2}+\left(a_{\mathrm{p}}^{*}-a_{\mathrm{v}}^{*}\right)^{2}+\left(b_{\mathrm{p}}^{*}-b_{\mathrm{v}}^{*}\right)^{2}}$

Mit dieser Formel kann der Farbunterschied $\Delta E$ zwischen dem Farbton $\mathrm{p}\left(\mathrm{L}^{*} \mathrm{a}^{*} \mathrm{~b}^{*}\right)$ und dem Farbton $v\left(L^{*} a^{*} b^{*}\right)$ berechnet werden (Commission Internationale de l'Eclairage (CIE) 2004).

Der $\Delta$ E-Schwellenwert, der für diese Studie interessant ist, da ab diesem ein Farbunterschied mit dem menschlichen Auge wahrnehmbar ist, liegt bei $\Delta \mathrm{E}=3,7$ (Johnston und Kao 1989). $\Delta \mathrm{E}=1$ entspricht einer geringen aber noch tolerierbaren Farbabweichung. $\mathrm{Ab}$ $\Delta \mathrm{E}=>5$ spricht man nicht mehr von einer Farbabweichung sondern von einer anderen Farbe.

Auch bei Spektrophotometern gibt es -wie bei jeder anderen Methode- den Einfluss von Messfehlern (Berns et al. 2000), insbesondere bei der Durchführung in vivo. Hierbei spielen die Faktoren des Umgebungslichtes, der Kopfhaltung bei der dentalen Farbbestimmung in Relation zum Messgerät sowie die Geschicklichkeit des Untersuchers eine Rolle (Knösel et al. 2009).

Die Reproduzierbarkeit kann durch Wiederholungsmessungen untersucht werden. Diese Untersuchung wurde auch in der vorliegenden Studie für die Methodenfehleranalyse angewandt.

Zudem wurde vor jeder Messung die Validität durch einen Kalibrierungsprozess (Messung eines definierten Farbfelds) abgesichert. 


\subsection{Studienablauf}

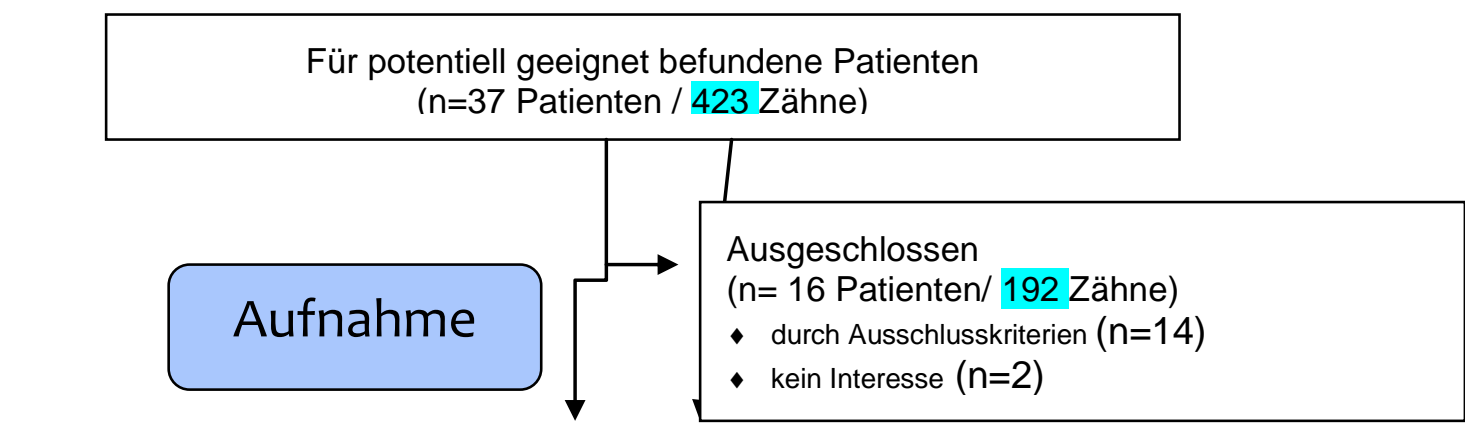

Randomisierung ( $n=21$ Patienten, $n=231$ Zähne; 82 Quadranten)

Zuweisung der Quadranten 1 und 3, oder 2 und 4, zur Behandlungs- (Icon) oder Kontrollgruppe.

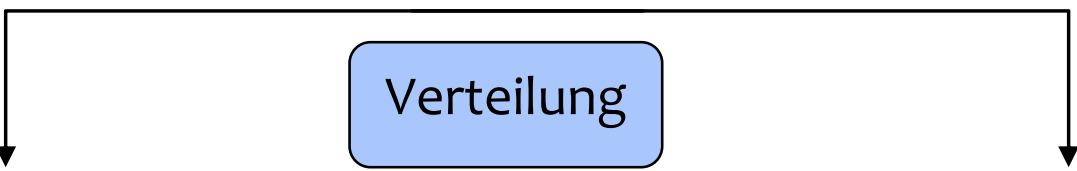

Zuweisung Behandlungsgruppe (Infiltration) ( $n=117$ Zähne/ 41 Quadranten: 11xQuadrant 1; 10xQuadrant 3; 10xQuadrant 2; 10xQuadrant 4;)

- Behandlung und Untersuchung durchgeführt ( $n=117$ Zähne/ 41 Quadranten)
Zuweisung Kontrollgruppe

( $\mathrm{n}=114$ Zähne / 41 Quadranten: 11xQuadrant 1; 10xQuadrant 3; 10xQuadrant 2; 10xQuadrant 4;)

- Untersuchung durchgeführt ( $n=114$ Zähne / 41 Quadranten)

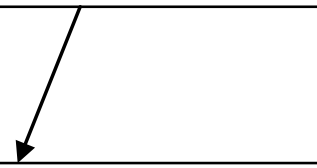

- Ausgeschlossen ( $\mathrm{n}=1$ Patient / 6 Zähne / 2 Quadranten: 1x Quadrant 1, 1x Quadrant 3; Grund: Patient erschien zu keinem der Kontrolltermine

\section{Nachkontrolle}

\section{Auswertung}

- Ausgeschieden ( $\mathrm{n}=1$ Patient / 6 Zähne / 2 Quadranten: 1x Quadrant 2, 1x Quadrant 4; Grund: Patient erschien zu keinem der Kontrolltermine

\section{Ausgewertet}

( $\mathrm{n}=20$ Patienten / 111 Zähne / 39 Quadranten: 10xQuadrant 1, 9xQuadrant 3, 10xQuadrant 2, 10xQuadrant 4)

- Von der Auswertung ausgeschlossen:

(t1: $\mathrm{n}=1$ Zahn nicht auswertbar,

t2: $n=1$ Patient / 6 Zähne; nicht zur t2-Kontrolle erschienen

t4: $n=1$ Patient / 6 Zähne; nicht zur t4-Kontrolle erschienen)
Ausgewertet

( $\mathrm{n}=20$ Patienten /108 Zähne / 39 Quadranten: 10xQuadrant1, 10xQuadrant 3, 10xQuadrant 2, 10xQuadrant 4)

- Von der Auswertung ausgeschlossen:

(t1: $\mathrm{n}=3$ Zähne nicht auswertbar,

t2: $n=1$ Patient / 6 Zähne; nicht zur t2-Kontrolle erschienen

t4: $n=1$ Patient / 6 Zähne; nicht zur t4-Kontrolle erschienen) 
Im Anschluss an die Genehmigung der Studie durch die Ethikkommisson der Universitätsmedizin Göttingen (siehe Anhang, Seite 85) wurden am Klinikum der Universitätsmedizin Göttingen über ein Jahr in Frage kommende Patienten auf die Möglichkeit einer Teilnahme an der Studie angesprochen und gegebenenfalls akquiriert. Von 37 in diesem Zeitraum potentiell geeigneten Patienten mit WSL, während bzw. nach Debonding, kamen nach Berücksichtigung der Ausschlusskriterien 23 Patienten als für die Studie geeignet in Betracht. (siehe Fließdiagramm) Zwei Patienten waren an einer Teilnahme nicht interessiert. Somit begann die Studie mit 21 kieferorthopädisch behandelten Patienten ( $n=21$ Patienten, $m=10 / f=11$, mittleres Alter 15,5; $n=231$ Zähne; 82 Quadranten), die nach Multibracketbehandlung entsprechende Entmineralisierungen an den Zähnen aufwiesen. Die Befundung der WSL erfolgte durch die Doktorandin / Zahnärztin (Amely Eckstein) in Konsultation eines Fachzahnarztes für Kieferorthopädie (PD Dr. Michael Knösel). Die Behandlung wurde in einem kontrollierten randomisierten Split-Mouth-Design an den Frontzähnen durchgeführt. Hierbei dienten entweder der erste und dritte Quadrant (Zähne: 13, 12, 11, 33, 32, 31) oder der zweite und vierte Quadrant (Zähne: 23, 22, 21, 43, 42, 41) als behandelte Referenzgruppe. Die jeweils verbliebenen Quadranten stellten die Kontrollgruppe dar. Zur Randomisierung wurden die zu behandelnden Quadranten durch Würfeln anhand der Patientennummern im Vorfeld ausgewürfelt. Die ungeraden Zahlen 1, 3 und 5 standen für den 1. und 3. Quadranten und die geraden Zahlen 2, 4 und 6 bedeuteten, dass der 2. und 4. Quadrant infiltriert wurde.

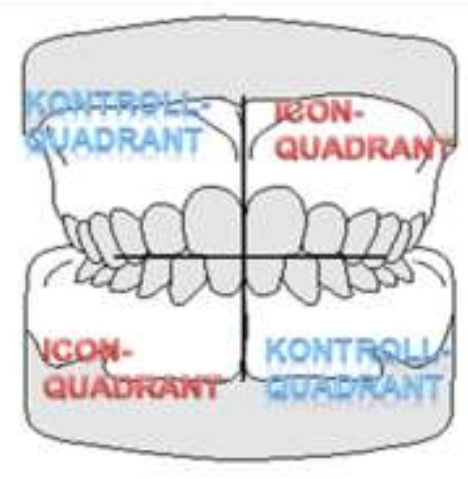

\section{Abbildung 8:}

Beispiel für Quadrantenzuweisung: Eine Behandlung erfolgt hier im zweiten und vierten Quadranten; die Quadranten eins und drei fungieren als Kontrollquadranten.

Nach ausführlicher Aufklärung über Risiken und mögliche Nebenwirkungen (Patientenaufklärungsbögen und Einverständniserklärungsformulare siehe Anhang S.79-84), erfolgte die Bestimmung der Farbe und Helligkeit (CIE-L* $a^{*} b^{*}$-Daten) der WSL und des angrenzenden gesunden Schmelzbereichs (AGS). Die absoluten CIE-L*a*b*-Daten der einzelnen Flächen (AGS Kontrolle, AGS Icon, WSL Kontrolle und WSL Icon) wurden aufgezeichnet, die Differenzen zwischen AGS und WSL gebildet und in Relation zu den Differenzen der korrespondierenden Flächen auf den Kontrollzähnen gesetzt. Für die 
Farbbestimmung kam das unter 4.2.1. und 4.2.3. beschriebene Spektrophotometer (ShadePilot, Degudent, Hanau-Wolfgang, Deutschland) zum Einsatz. Im Vorfeld der Studie erfolgte ein Training zur sicheren Handhabung des Gerätes an neun freiwilligen Probanden. Nach Abschluss der Messung an 13 Studien-Patienten erfolgte eine statistische Vorauswertung und Gruppengrößenkalkulation, um die finale Gruppengröße auf Basis der Unterschiede zwischen WSL und AGS festzulegen. Die Gruppengröße wurde für eine Power von mindestens $80 \%$ bei einer Auflösung von möglichen signifikanten Unterschieden (t0 vs. t5) bei einem Schwellenwert von $\Delta \mathrm{E}=3,0$ Einheiten auf einem Signifikanzniveau von alpha $=5 \%$ adjustiert. Der Schwellenwert von CIE- $L^{*} a^{*} b^{*}-\Delta E=3,0$ Einheiten ist in der Literatur als Untergrenze der in einem klinischen Setting mit bloßem Auge differenzierbaren Farbnuancen angegeben (Archegas et al. 2011). Anderen Untersuchungen zufolge liegt der Schwellenwert der klinischen Sichtbarkeit sogar bei $\Delta \mathrm{E}=3,3$ Einheiten (Inokoshi 1996, Kim und Um 1996, Ruyter et al. 1987) oder bei $\Delta \mathrm{E}=3,7$ Einheiten (Johnston und Kao 1989).

Basierend auf der kompletten Beurteilung der ersten 13 Patienten konnte errechnet werden, dass für ein aussagekräftiges Ergebnis 20 Patienten (jeder mit im Mittel $n=8.3$ gemessenen Zähnen) benötigt wurden.

Von den vorher mit Multibracketapparaturen behandelten 21 Patienten im Alter von 12 bis 19 Jahren (mittleres Alter 15,5 J) sind 21 (10 männliche, 11 weibliche) mit WSL ( $\mathrm{n}=231$ Zähne; 114 Kontrollzähne / 117 Behandelte Zähne je 41 Quadranten) untersucht worden. Davon wurden 117 Zähne mit Icon behandelt, 114 Zähne wurden der Kontrollgruppe zugewiesen. Ausschlusskriterien stellten eingebrochene Läsionen, sowie Füllungen, restaurierte Zähne und Milchzähne dar. Es kam nach vorgenommener Infiltration zum Ausschluss eines Patienten ( $\mathrm{n}=12$ Zähne, 6 Kontollzähne/2 Quadranten; 6 Behandelte Zähne/2 Quadranten), da er zu keinem der Folgetermine erschien. Die Studie konnte somit mit $n=20$ Patienten ( $\mathrm{n}=219 Z$ Zähne; 111 Behandelte Zähne/39Quadranten; 108 Kontrollzähne/39 Quadranten) durchgeführt und planmäßig beendet werden. Tabelle I (Seite 32) zeigt detaillierte Informationen und Zeiträume zur kieferorthopädischen Behandlung, Beginn und Ablauf der Infiltrationsbehandlung und zu den Folgeuntersuchungen. 


\subsubsection{Behandlungsablauf}

Die Erziehungsberechtigten und die Patienten wurden umfassend mündlich und schriftlich aufgeklärt und gaben ihre schriftliche Zustimmung. (Aufklärungsbögen im Anhang S. 79-84). Vor der Behandlung wurden die Zähne mit fluoridfreier Polierpaste („Zircate prophy paste“, Dentsply DeTrey $\mathrm{GmbH}$, Konstanz, Deuschland) gereinigt und anschließend erfolgten mit der Digitalkamera des Spektrophotometers (ShadePilot, Degudent, Hanau-Wolfgang, Deutschland) Aufnahmen der Untersuchungszähne 13 bis 23 im Oberkiefer und 33 bis 43 im Unterkiefer) (Baseline, t0). Zum Schutz der Gingiva kamen unter die freie Gingiva gelegte, ungetränkte Retraktionsfäden („Ultrapak“, Größe 0, Ultradent, Utha, USA) zum Einsatz, um diese vor dem Kontakt mit dem $\mathrm{HCl}-\mathrm{Gel} z u$ isolieren (Abbildung 9a und b).

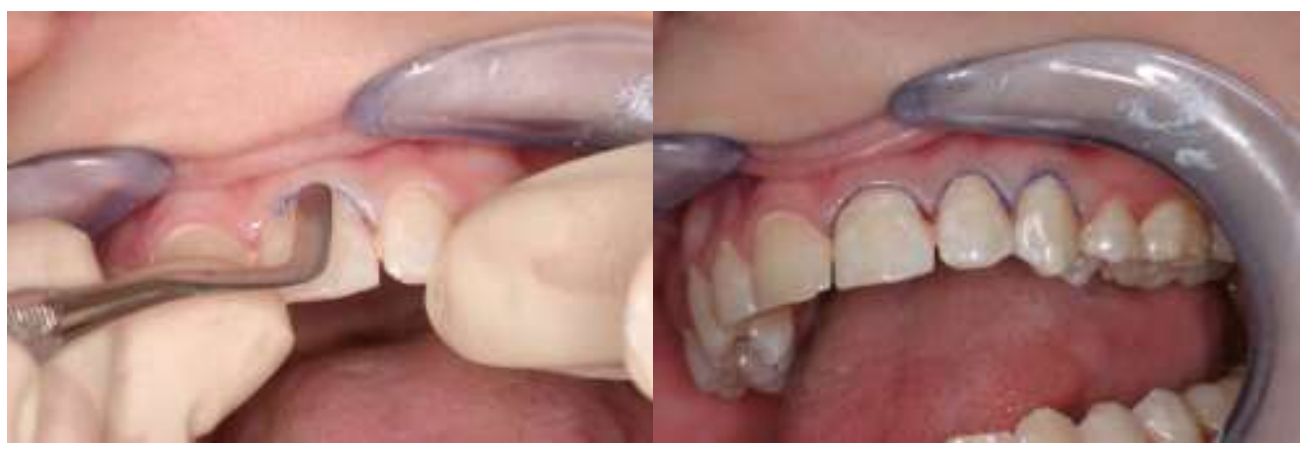

Abbildung 9a und b: Legen der Retraktionsfäden

Um die Zähne trocken zu legen, wurde ein Wangenabhalter eingesetzt und ein Sauger und Watte eingebracht. Als erstes wurde Icon-Etch, das 15\%ige $\mathrm{HCl}-\mathrm{Gel}$, nach Herstellerangaben auf die Läsionsstellen der betroffenen Zähne aufgetragen (Abbildung 9c), nach zwei Minuten Einwirkzeit wieder abgespült und mit Luft getrocknet.

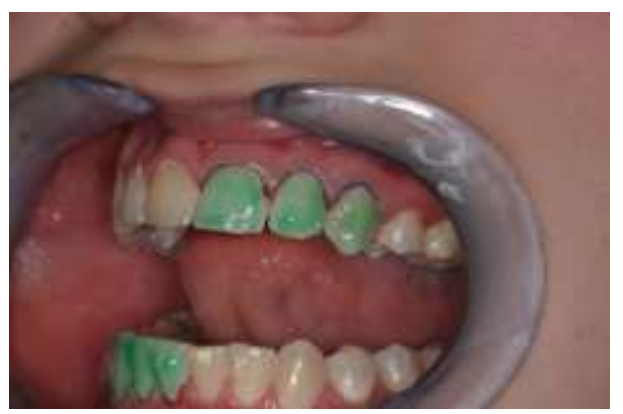

Abbildung 9c:

Das Anrauen mit dem Icon-Etch

Dieser Vorgang der Konditionierung konnte bis zu vier Mal wiederholt werden, um die pseudointakte Oberfläche der Läsionen zu entfernen und somit eine Infiltration zu ermöglichen (Meyer-Lueckel et al. 2007). 
In Einhaltung der Anleitung wurden also die Ätzintervalle an das Ausmaß der WSL angepasst. Bei stärker ausgeprägten WSL erfolgte eine häufigere Schmelzätzung als bei kleinen Läsionen. Über die Notwendigkeit weiterer Ätzvorgänge wurde nach visueller Kontrolle entschieden. Mit dem Aufbringen der Dry-Lösung kann man den späteren ästhetischen Angleichungseffekt gut voraussehen. Gleicht sich die WSL-Farbe der Zahnfarbe beim Benutzen der Dry-Lösung kurz an, so bedeutet das eine vollständige Entfernung der pseudointakten Schicht; weitere Ätzvorgänge sind nicht notwendig. Ist das nicht der Fall, erfolgen weitere Ätzvorgänge. Es zeigte sich, dass vor allem bei älteren und ausgeprägteren WSL eine längere Ätzdauer zu ästhetisch besseren Endergebnissen führt. Die Anzahl der durchgeführten Ätzvorgänge pro Patient ist aus der Tabelle I (Seite 32) ersichtlich.

Als nächstes wurde Icon-Dry auf die Läsionsstellen aufgetragen, um 30 Sekunden einzuwirken und anschließend mit Luft getrocknet zu werden. (Abbildung 9d und e)

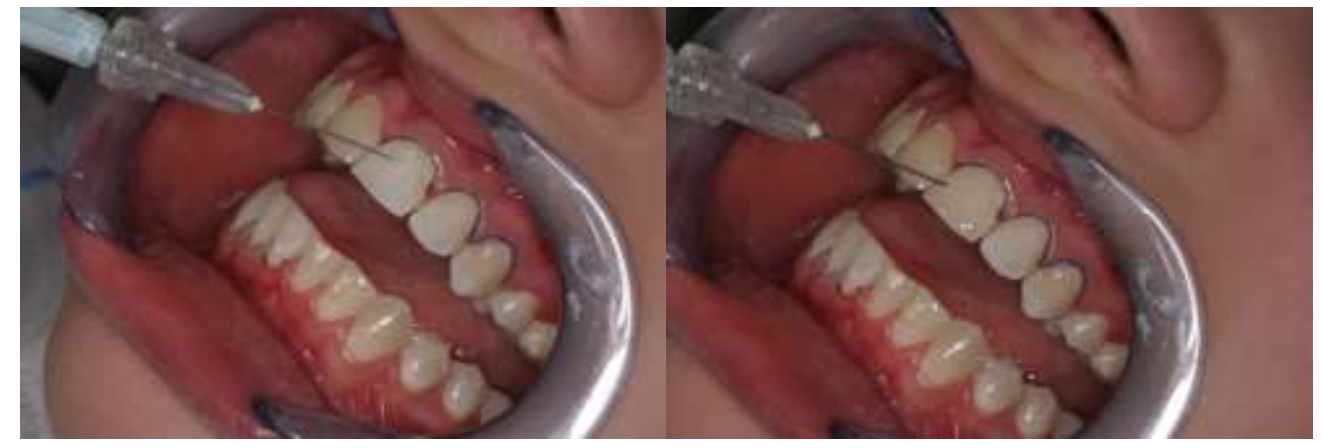

Abbildung 9d und e: Das Auftragen des Icon-Dry

Auf die komplett trockene, vorbereitete Schmelzoberfläche wurde nun der Icon-Infiltrant aufgetragen (Abbildung 9f) und nach einer Infiltrationszeit von drei Minuten für 40 Sekunden lichtgehärtet Abbildung 9g). Dieser Vorgang erfolgte im Anschluss ein zweites Mal mit einer Minute Einwirkzeit.

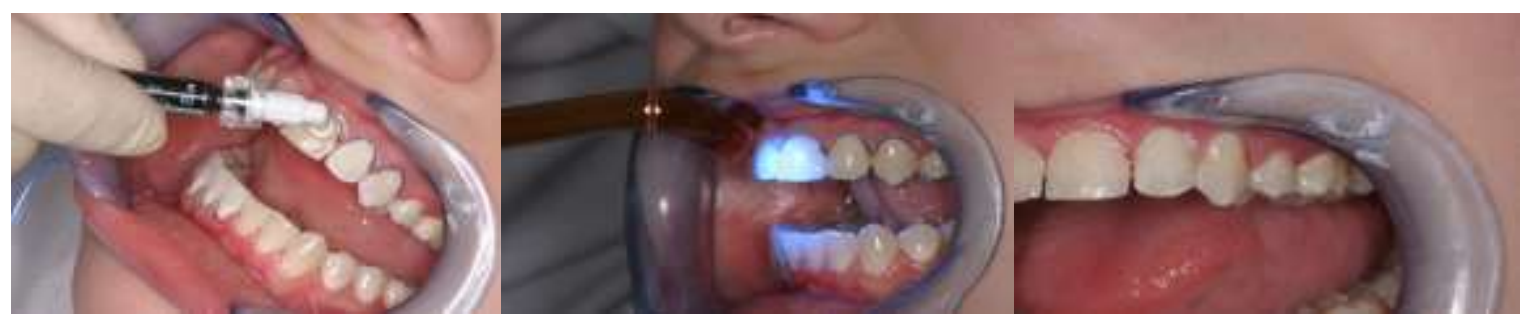

Abbildung 9f: Auftragen des Icon-Infiltranten

Abbildung 9g: Lichtaushärtung

Abbildung 9h: Abschlusssituation 


\begin{tabular}{|c|c|c|c|c|c|c|}
\hline $\begin{array}{c}\text { Patient } \\
\#\end{array}$ & $\begin{array}{l}\text { Komplette } \\
\text { Ätzdauer } \\
\text { (Minuten) }\end{array}$ & $\begin{array}{l}\text { Zeitraum zw. } \\
\text { Entbänderung } \\
\text { und Infiltration } \\
\text { (Monate) }\end{array}$ & $\begin{array}{l}\text { Versuchs- } \\
\text { zähne } \\
\text { (n) }\end{array}$ & $\begin{array}{l}\text { Icon- } \\
\text { Zähne } \\
\text { (n) }\end{array}$ & $\begin{array}{l}\text { Kontroll- } \\
\text { Zähne (n) }\end{array}$ & $\begin{array}{l}\text { Fehlende } \\
\text { Messungen } \\
\text { (Zeitpunkte) }\end{array}$ \\
\hline 1 & 6 & 8 & 8 & 4 & 4 & --- \\
\hline 2 & 6 & 3 & 11 & 5 & 6 & --- \\
\hline 3 & 6 & 5 & 12 & 6 & 6 & $\begin{array}{l}\text { t1: vier } \\
\text { Bilder nicht } \\
\text { beurteilbar }\end{array}$ \\
\hline 4 & 6 & 5 & 11 & 5 & 6 & --- \\
\hline 5 & 6 & 11 & 12 & 6 & 6 & t4 \\
\hline 6 & 6 & 9 & 12 & 6 & 6 & --- \\
\hline 7 & 6 & 8 & 12 & 6 & 6 & t2 \\
\hline 8 & 6 & 12 & 12 & 6 & 6 & --- \\
\hline 9 & 8 & 6 & 12 & 6 & 6 & --- \\
\hline 10 & 6 & 9 & 12 & 6 & 6 & --- \\
\hline 11 & 8 & 7 & 11 & 6 & 5 & --- \\
\hline 12 & 8 & 9 & 12 & 6 & 6 & t2 \\
\hline 13 & 6 & 3 & 12 & 6 & 6 & $\mathrm{t} 4, \mathrm{t} 5$ \\
\hline 14 & 7 & 1 & 8 & 4 & 4 & --- \\
\hline 15 & 7 & 1 & 12 & 6 & 6 & --- \\
\hline 16 & 7 & 1 & 11 & 6 & 5 & --- \\
\hline 17 & 8 & 4 & 12 & 6 & 6 & --- \\
\hline 18 & 8 & 1 & 12 & 6 & 6 & --- \\
\hline 19 & 8 & 2 & 7 & 5 & 2 & --- \\
\hline 20 & 8 & 1 & 8 & 4 & 4 & --- \\
\hline \multirow[t]{2}{*}{21} & 6 & 1 & $\begin{array}{l}\text { ausge- } \\
\text { schlossen }\end{array}$ & $\begin{array}{l}\text { ausge- } \\
\text { schlossen }\end{array}$ & $\begin{array}{l}\text { ausge- } \\
\text { schlossen }\end{array}$ & $\mathrm{t} 2, \mathrm{t} 3, \mathrm{t} 4, \mathrm{t} 5$ \\
\hline & & & Total: 219 & Total: 111 & Total: 108 & \\
\hline
\end{tabular}

Tabelle I: Detaillierte Informationen und Zeiträume zu kieferorthopädischer Behandlung, Beginn und Ablauf der Infiltrationsbehandlung und den Folgeuntersuchungen.

Das Schmelzätzen wurde in Zwei-Minuten-Intervallen durchgeführt. In drei Fällen erfolgte die Konditionierung beim letzten Vorgang für nur eine Minute (Patient 14-16). Die DurchschnittsÄtzzeit betrug 6,8 Minuten. Durchschnittlich wurde die Inflitration 5,1 Monate nach der Entbänderung durchgeführt. 
Da bei Kariesinfiltration keine Kunststoffschicht auf dem Läsionskörper für einen effektiven Verschluss notwendig ist, kann etwaiges überschüssiges Material vor der Lichthärtung entfernt werden (Paris und Meyer-Lueckel 2010).

Zum Abschluss wurden die Zähne noch einmal mit Polierpaste („Zircate prophy paste“, Dentsply DeTrey $\mathrm{GmbH}$, Konstanz, Deutschland) poliert und die Zwischenräume mit Zahnseide gereinigt. Die t1-Aufnahmen erfolgten nach beendeter Infiltration (Abbildung 9h) mit dem Spektrophotometer (ShadePilot, Degudent, Hanau-Wolfgang, Deutschland) (t1). Die weiteren Bilder wurden dann nach einer Woche (t2), nach einem Monat (t3), nach drei Monaten (t4) und nach einem halben Jahr (t5) wiederholt. Nach einem halben Jahr wurden im Anschluss an die t5-Messung bei allen Patienten auch die Zähne der Kontrollgruppe mit Icon versiegelt.

Für die komplette Laufzeit der Studie wurden die Patienten mit den gleichen Zahnbürsten („Oral-B Classic Care“, Procter \& Gamble, Schwalbach am Taunus, Deutschland) und Zahnpasten („elmex“, GABA, Lörrach, Deutschland) versorgt, um die Voraussetzungen für die Studienteilnehmer weitestgehend anzugleichen.

Für die Auswertung der mit dem ShadePilot aufgenommenen Bilder kam auf dem Computer das dazugehörigen ShadePilot-Programm (DeguDent Shadepilot, Hanau-Wolfgang, Deutschland) von Degudent (Hanau, Deutschland) zum Einsatz. Es erfolgte jeweils die Messung der $L^{*} a^{*} b^{*}$-Werte einer reproduzierbaren Stelle im gesunden Schmelz und einer ebenfalls reproduzierbaren Stelle im White-Spot an jedem Zahn. Die Messareale wurden für jeden Patienten und jeden Zahn auf dem Computer gespeichert, um ein Wiederauffinden der maskierten WSL zu ermöglichen und um zu gewährleisten, dass die Messungen an exakt denselben Arealen erfolgte. So konnte die Veränderung der Farbe in den WSL mit IconBehandlung und in den WSL ohne Icon-Behandlung verglichen werden sowie der Farbangleich der WSL nach Icon-Behandlung an die Farbe des gesunden Schmelzes des jeweiligen Zahnes. 


\subsection{Statistische Analyse}

Die Farb- und Lichtwerte (CIE-L*a*b*) wurden jeweils für den WSL-Bereich und den angrenzenden Schmelz in jeder Gruppe zu den unterschiedlichen Zeitpunkten summiert und als $\Delta \mathrm{E}$-Werte berechnet:

$\Delta \mathrm{E}_{(\operatorname{Par} 1-\operatorname{Par} 2)}=\left[\left(\mathrm{L}_{\text {Par } 1}-\mathrm{L}_{\text {Par 2 }}\right)^{2}+\left(\mathrm{a}_{\text {Par } 1}-\mathrm{a}_{\text {Par 2 }}\right)^{2}+\left(\mathrm{b}_{\text {Par } 1}-\mathrm{b}_{\text {Par } 2}\right)^{2}\right]^{1 / 2}$

Untersuchungsparameter ('PAR') repräsentieren in dieser Formel jeweils entweder dieselbe Fläche zu unterschiedlichen Zeitpunkten oder verschiedene Flächen zum selben Zeitpunkt.

Es wurden sowohl die WSL- und AGS-Flächen mit den entsprechenden Flächen der Kontroll-Gruppe verglichen (Inter-Gruppenvergleich) als auch die Entwicklung der einzelnen Flächen separat in den Gruppen untersucht. Weiterhin wurden die Unterschiede zwischen WSL und AGS auf signifikante Unterschiede getestet (Intra-Gruppenvergleich), um die Entwicklung der Farbunterschiede über die Zeit möglichst vollständig abzubilden. Dabei wurden sowohl die Entwicklung der Farb- und Helligkeitswerte separat als auch die summierten $\Delta \mathrm{E}$-Farb- und Helligkeitswerte dargestellt, um die klinische Sichtbarkeit der Veränderungen beurteilen zu können (Johnston und Kao 1989).

Für die Licht- und Farbparameter $L^{*}, a^{*}$ und $b^{*}$ fand die Kalkulation der Mittelwerte und des 95\% Konfidenzintervalls separat für die WSL und den umgebenden Schmelz statt.

Für die Auswertung kam eine multifaktorielle Varianzanalyse mit wiederholten Messungen (repeated measures) zum Einsatz (F1-LDF1 ANOVA). Ein Faktor war die "Infiltrationsbehandlung" (F1), der zweite Faktor die wiederholten Messungen über die Zeit (Versuchszeitspanne, longitudinal (LDF1). Dann wurden die Gruppen ohne und mit Behandlung hinsichtlich des globalen Zeitverlaufs getrennt untersucht. Abschließend wurden alle Zeitpunkte in beiden Gruppen getrennt paarweise miteinander verglichen (15 = Anzahl der Paarvergleiche/Patienten). So konnten die Effekte der Infiltration und des Zeitverlaufs auf die Farbunterschiede ( $\Delta$-E Werte) analysiert werden.

Die Patienten wurden als randomisierter Faktor betrachtet. Im Fall von signifikanten Interaktionen erfolgten paarweise Vergleiche für alle Versuchszeitpunkte mit BonferroniJustierung des a-levels. Das Signifikanzniveau wurde mit $\alpha=5 \%$ festgelegt. Für die statistische Analyse kam SAS 9.3 (SAS Institute; Cary, NC, USA) und Statistica (StatSoft (europe) $\mathrm{GmbH}$; Hamburg, Deutschland) zum Einsatz. 


\subsection{Methodenfehlerbestimmung}

Ein Vorversuch diente zur Bestimmung des Methodenfehlers. Dabei wurden in vivo jeweils die $C I E-L^{*} a^{*} b^{*}-W e r t e$ von einem oberen und einem unteren zentralen Inzisivius zehn Mal an zehn aufeinanderfolgenden Tagen aufgenommen. Die Durchführung der Messung erfolgte erst durch die Doktorandin und direkt im Anschluss durch einen unabhängigen Untersucher. Die obere und untere Grenze der Abweichungen (Methodenfehler) lag zwischen 0.16 Einheiten ( $L^{*}-$ Wert, oberer Inzisivus) und 0.82 Einheiten ( $a^{*}$-Wert, oberer Inzisivus). Des Weiteren wurde die Korrelation der $\mathrm{a}^{*} / \mathrm{b}^{*}$-Werte bei t0 und $\mathrm{t} 5$ ermittelt, um die Genauigkeit der gemessenen Farbwerte zu testen (Knösel et al. 2012a). Diese sollte auch bei Farb- und Helligkeitsveränderungen konstant bleiben. So ließ sich zusätzlich die Messgenauigkeit überprüfen.

Bei t0 war die $a^{*} / b^{*}$-Korrelation $r=0.42(p<0.0001)$ für WSL und $r=0.52(p<0.0001)$ für AGS. Bei t5 war die Korrelationsstruktur fast identisch: $r=0.4(p<0.0001)$ für WSL und $r=0.53$ $(p<0.0001)$ für AGS, weswegen die gemessenen Farbwerte als valide einzustufen sind.

Diese Ergebnisse stimmen mit vorherigen Ergebnissen in Bezug auf die Genauigkeit eines Spektrophotometers überein (Sluzker et al. 2011) und zeigen eine Präzision, die weit über der Kapazität des menschlichen Auges liegt (Johnston and Kao 1989).

In der folgenden Tabelle sind die detaillierten Informationen der Methodenfehleranalyse aufgelistet.

\begin{tabular}{|c|c|c|c|c|c|c|c|}
\hline \multicolumn{8}{|c|}{ Oberer rechter Inzisivus (11) } \\
\hline Parameter & Untersucher & $\mathrm{N}$ & Mittelwert & Min. & Max. & Spanne & SD \\
\hline L & 1 & 10 & 79.86 & 79.76 & 79.98 & 0.22 & 0.077 \\
\hline$A$ & 1 & 10 & 2.91 & 2.50 & 3.32 & 0.82 & 0.28 \\
\hline B & 1 & 10 & 14.34 & 14.10 & 14.50 & 0.40 & 0.17 \\
\hline L & 2 & 10 & 79.79 & 79.74 & 79.90 & 0.16 & 0.05 \\
\hline A & 2 & 10 & 2.83 & 2.52 & 3.20 & 0.68 & 0.23 \\
\hline$B$ & 2 & 10 & 14.32 & 14.10 & 14.50 & 0.40 & 0.11 \\
\hline \multicolumn{8}{|c|}{ Unterer rechter Inzisivus (41) } \\
\hline L & 1 & 10 & 73.47 & 73.40 & 73.58 & 0.18 & 0.05 \\
\hline A & 1 & 10 & 3.13 & 2.81 & 3.57 & 0.76 & 0.32 \\
\hline B & 1 & 10 & 13.53 & 13.26 & 13.66 & 0.40 & 0.15 \\
\hline$L$ & 2 & 10 & 73.41 & 73.35 & 73.59 & 0.24 & 0.08 \\
\hline A & 2 & 10 & 3.20 & 2.80 & 3.57 & 0.77 & 0.28 \\
\hline B & 2 & 10 & 13.493 & 13.26 & 13.66 & 0.40 & 0.1609 \\
\hline
\end{tabular}

Tabelle II: detaillierte Ergebnisse der Methodenfehleranalyse 


\section{Ergebnisse}

Die Baseline- $\Delta \mathrm{E}-$ Werte der Kontrollzähne und der zu behandelnden Zähne wurden verglichen, wobei keine signifikanten Unterschiede in Farb- und Lichtcharakteristika gefunden wurden, so dass eine Vergleichbarkeit der Gruppen gegeben ist.

Die Varianzanalyse ergab hoch signifikante Unterschiede im zeitlichen Verlauf zwischen infiltrierten und Kontrollzähnen (Inter-Gruppenvergleich).

\section{Entwicklung der summierten Farb-und Helligkeitsunterschiede}

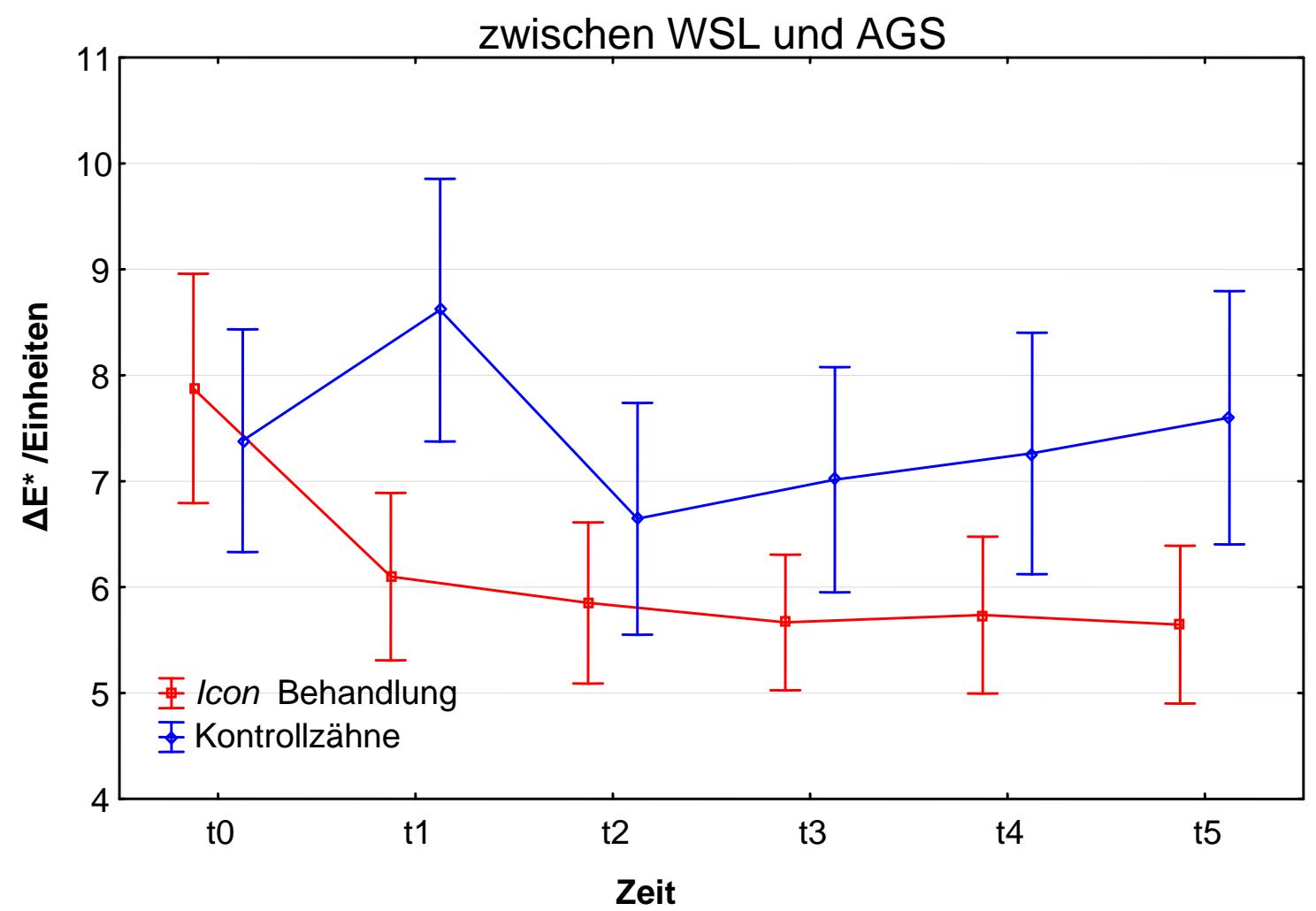

Abbildung 10: Entwicklung der summierten Farb- und Helligkeitsunterschiede zwischen den WSL und dem umliegendem Schmelz. Die Grafik zeigt die mittlere Differenz zwischen WSL und AGS bei den infiltrierten und den Kontrollzähnen. Diese weist einen Abfall in der IconGruppe nach Infiltration auf (t1), der sich über sechs Monate nicht signifikant verändert.

Der temporäre Anstieg der Helligkeit in der Kontrollgruppe bei $\mathrm{t} 1$ ist auf das Trockenlegen der Zahnreihen während der Behandlung zurückzuführen. 


\begin{tabular}{|c|c|c|c|c|c|c|}
\hline \multirow{11}{*}{ to } & Parameter & Bereich & $\begin{array}{l}\text { Mittelwert- } \\
\text { Icon } \pm \text { SD }\end{array}$ & $\begin{array}{l}\text { Mittelwert- } \\
\text { Kontrollgruppe } \pm \text { SD }\end{array}$ & $\begin{array}{l}\mathrm{N} \text {-Icon / } \\
\mathrm{N}-\text { Kontroll- } \\
\text { gruppe }\end{array}$ & P-Wert \\
\hline & L & WSL & $72.84 \pm 4.21$ & $73.12 \pm 3.81$ & $105 \mid 101$ & 0.62 \\
\hline & $\mathrm{L}$ & AGS & $72.59 \pm 2.46$ & $72.46 \pm 2.64$ & $111 \mid 108$ & 0.71 \\
\hline & A & WSL & $6.36 \pm 2.49$ & $5.85 \pm 2.69$ & $104 \mid 101$ & 0.16 \\
\hline & $A$ & AGS & $5.43 \pm 1.41$ & $5.63 \pm 1.65$ & $111 \mid 108$ & 0.33 \\
\hline & B & WSL & $17.89 \pm 7.37$ & $16.59 \pm 6.99$ & $104 \mid 101$ & 0.20 \\
\hline & B & AGS & $20.72 \pm 3.18$ & $20.76 \pm 3.25$ & $111 \mid 108$ & 0.92 \\
\hline & $\Delta \mathrm{L}$ & WSL/AGS & $0.17 \pm 3.72$ & $0.56 \pm 3.92$ & $105 \mid 101$ & 0.47 \\
\hline & $\Delta \mathrm{a}$ & WSL/AGS & $1 \pm 2.5$ & $0.44 \pm 2.65$ & $104 \mid 101$ & 0.13 \\
\hline & $\Delta b$ & WSL/AGS & $-2.94 \pm 7.42$ & $-4.22 \pm 7.18$ & $104 \mid 101$ & 0.21 \\
\hline & $\Delta \mathrm{E}(\mathrm{L}, \mathrm{a}, \mathrm{b})$ & WSL/AGS & $7.88 \pm 4.44$ & $7.38 \pm 4.34$ & $67 \mid 68$ & 0.52 \\
\hline \multirow{10}{*}{ t5 } & L & WSL & $70.64 \pm 3.31$ & $72.78 \pm 4.16$ & $95 \mid 92$ & $<0.001$ \\
\hline & L & AGS & $72.4 \pm 2.4$ & $72.42 \pm 2.51$ & $102 \mid 97$ & 0.96 \\
\hline & A & WSL & $7.18 \pm 2.72$ & $5.96 \pm 2.75$ & $95 \mid 92$ & 0.003 \\
\hline & $A$ & AGS & $5.38 \pm 1.62$ & $5.7 \pm 1.83$ & $102 \mid 97$ & 0.20 \\
\hline & B & WSL & $20.94 \pm 3.52$ & $17.88 \pm 7.59$ & $95 \mid 92$ & 0.001 \\
\hline & B & AGS & $22.19 \pm 3.28$ & $21.42 \pm 3.31$ & $102 \mid 97$ & 0.101 \\
\hline & $\Delta \mathrm{L}$ & WSL/AGS & $-1.84 \pm 3.01$ & $0.14 \pm 3.96$ & $95 \mid 90$ & $<0.001$ \\
\hline & $\Delta a$ & WSL/AGS & $1.95 \pm 2.46$ & $0.5 \pm 2.66$ & $95 \mid 90$ & $<0.001$ \\
\hline & $\Delta \mathrm{b}$ & WSL/AGS & $-1.32 \pm 3.53$ & $-3.55 \pm 7.8$ & $95 \mid 90$ & 0.01 \\
\hline & $\Delta \mathrm{E}(\mathrm{L}, \mathrm{a}, \mathrm{b})$ & WSL/AGS & $5.65 \pm 2.83$ & $7.6 \pm 4.51$ & $58 \mid 57$ & 0.01 \\
\hline
\end{tabular}

Tabelle III: Bei dem Inter-Gruppen-Vergleich wurden in beiden Gruppen die summierten $\mathrm{L}^{*} / \mathrm{a}^{*} / \mathrm{b}^{*}$-Werte $(\Delta \mathrm{E})$ zum Zeitpunkt Baseline (t0) und nach sechs Monaten (t5) betrachtet. Der Farb- und Helligkeitsunterschied der WSL und AGS sind zur Baseline weder in der Behandlungs- noch in der Kontrollgruppe signifikant unterschiedlich. 


\subsection{Kontrollzähne}

In der Kontrollgruppe zeichnete sich bei t1 ein deutlicher Anstieg der Helligkeit der WhiteSpots sowie der umliegenden Schmelzregion ab (Abbildung 10, Seite 36 und Abbildung 11). Dieser Helligkeitsanstieg ist auf die Austrocknung der Zähne zurückzuführen, die eine unvermeidliche Nebenwirkung des Lippen- und Wangenretraktors, des Saugers und der Watte ist, die während der Infiltrationssitzung benutzt wurden. (Russell 2000) Dieser Effekt war nach einer Woche verschwunden und der Wert auf sein normales Niveau zurückgekehrt (t2). Zwischen 0 (Baseline), t2, t3, t4 und t5 konnten keine signifikanten Helligkeitsunterschiede beobachtet werden. Es gab keine signifikanten Veränderungen der $\Delta E-W e r t e$ nach sechs Monaten. Nichts wies auf eine Minderung der summierten Farb- und Helligkeitsunterschiede von $\triangle E$-Werten zwischen WSL und AGS im Vergleich zu Baseline hin (Tabelle IV, Seite 39, Abbildung 10, Seite 36 und Abbildung 11).

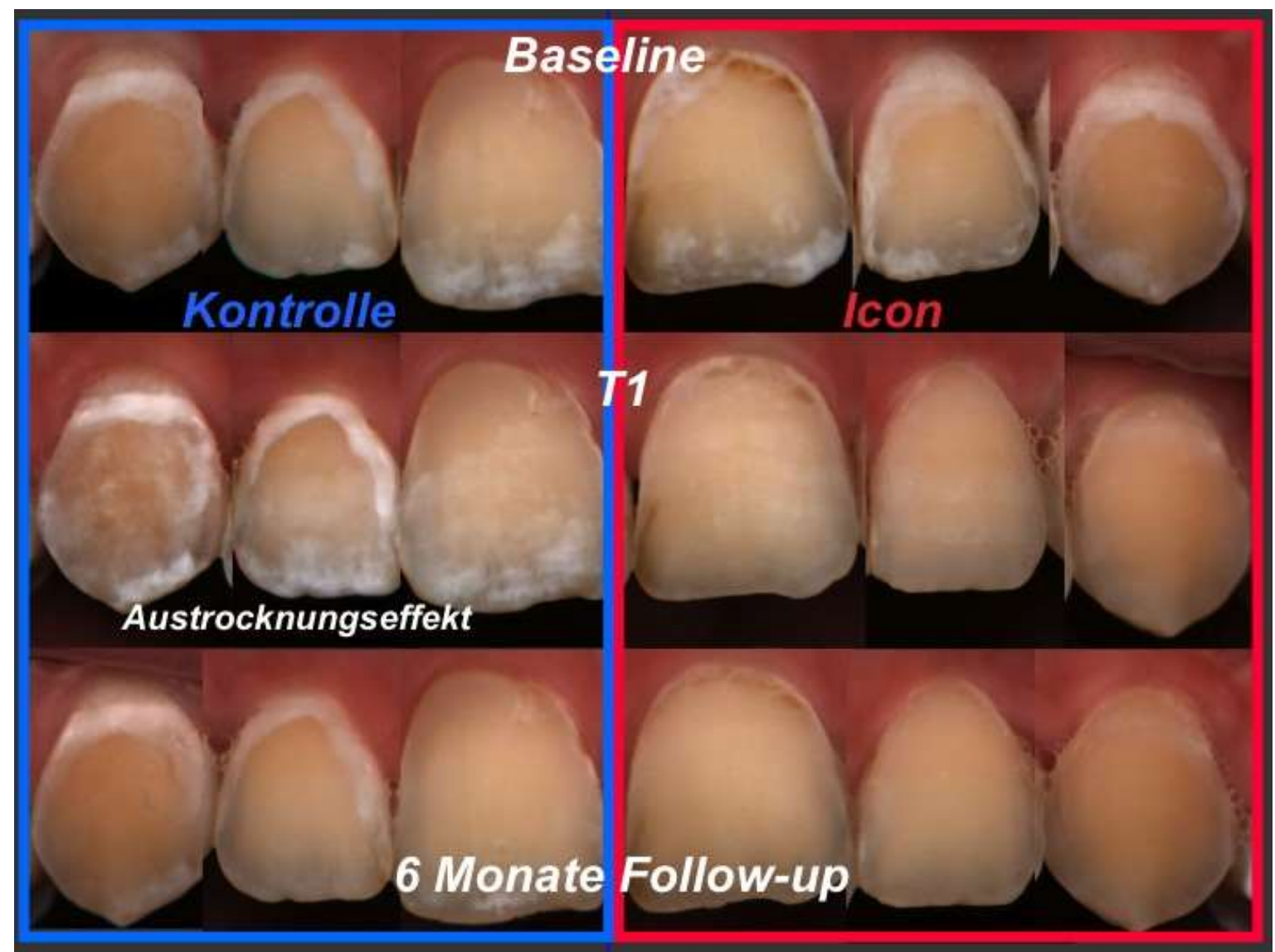

Abbildung 11: Fallbeispiel. Einzelaufnahmen spektrophotometrisch ausgewerteter Zähne zu den Zeitpunkten Baseline, t1 (nach Infiltration) und t5 (nach 6 Monaten). Der temporäre Aufhellungseffekt (Russell 2000) an den Kontrollzähnen bei t1 ist deutlich erkennbar. (Knösel et al. 2013 S.92) 


\begin{tabular}{|c|c|c|c|c|c|c|c|}
\hline $\begin{array}{l}\text { Gruppe/ } \\
\text { Bereich }\end{array}$ & $\begin{array}{l}\text { Para- } \\
\text { meter }\end{array}$ & $\begin{array}{l}\text { Mittelwert t0 } \\
\pm \mathrm{SD}\end{array}$ & $\begin{array}{l}\text { Mittelwert t5 } \\
\pm \mathrm{SD}\end{array}$ & $\begin{array}{l}\text { Diff. t0-t5 } \\
\pm \mathrm{SD}\end{array}$ & $\mathrm{N}$ & $\begin{array}{l}\text { KI 95\% Diff. } \\
\text { [min;max] }\end{array}$ & $P$ \\
\hline $\begin{array}{l}\text { Icon I } \\
\text { WSL }\end{array}$ & $\mathrm{L}$ & $72.73 \pm 4.27$ & $70.64 \pm 3.31$ & $2.09 \pm 3.63$ & 95 & {$[1.35 ; 2.83]$} & $<0.001$ \\
\hline $\begin{array}{l}\text { Kontroll } \\
\text { / WSL }\end{array}$ & $\mathrm{L}$ & $73.19 \pm 3.87$ & $72.78 \pm 4.16$ & $0.4 \pm 2.52$ & 92 & {$[-0.12 ; 0.93]$} & 0.13 \\
\hline $\begin{array}{l}\text { Icon I } \\
\text { AGS }\end{array}$ & $\mathrm{L}$ & $72.56 \pm 2.53$ & $72.4 \pm 2.4$ & $0.16 \pm 1.75$ & 102 & {$[-0.19 ; 0.5]$} & 0.36 \\
\hline $\begin{array}{l}\text { Kontroll } \\
\text { / AGS }\end{array}$ & $\mathrm{L}$ & $72.5 \pm 2.69$ & $72.42 \pm 2.51$ & $0.08 \pm 1.94$ & 97 & {$[-0.31 ; 0.47]$} & 0.68 \\
\hline $\begin{array}{l}\text { Icon I } \\
\text { WSL }\end{array}$ & A & $6.37 \pm 2.56$ & $7.17 \pm 2.74$ & $-0.79 \pm 1.95$ & 94 & {$[-1.19 ;-0.4]$} & $<0.001$ \\
\hline $\begin{array}{l}\text { Kontroll } \\
\text { / WSL }\end{array}$ & A & $5.75 \pm 2.74$ & $5.96 \pm 2.75$ & $-0.2 \pm 1.59$ & 92 & {$[-0.53 ; 0.13]$} & 0.23 \\
\hline $\begin{array}{l}\text { Icon I } \\
\text { AGS }\end{array}$ & A & $5.41 \pm 1.45$ & $5.38 \pm 1.62$ & $0.03 \pm 0.86$ & 102 & {$[-0.14 ; 0.2]$} & 0.72 \\
\hline $\begin{array}{l}\text { Kontroll } \\
\text { / AGS }\end{array}$ & A & $5.62 \pm 1.72$ & $5.7 \pm 1.83$ & $-0.08 \pm 0.89$ & 97 & {$[-0.26 ; 0.1]$} & 0.39 \\
\hline $\begin{array}{l}\text { Icon I } \\
\text { WSL }\end{array}$ & B & $17.75 \pm 7.5$ & $20.97 \pm 3.53$ & $-3.22 \pm 6.84$ & 94 & {$[-4.62 ;-1.82]$} & $<0.001$ \\
\hline $\begin{array}{l}\text { Kontroll } \\
\text { / WSL }\end{array}$ & B & $16.27 \pm 7.21$ & $17.88 \pm 7.59$ & $-1.6 \pm 4.17$ & 92 & {$[-2.47 ;-0.74]$} & $<0.001$ \\
\hline $\begin{array}{l}\text { Icon I } \\
\text { AGS }\end{array}$ & B & $20.57 \pm 3.15$ & $22.19 \pm 3.28$ & $-1.62 \pm 1.85$ & 102 & {$[-1.99 ;-1.26]$} & $<0.001$ \\
\hline $\begin{array}{l}\text { Kontroll } \\
\text { / AGS }\end{array}$ & B & $20.68 \pm 3.26$ & $21.42 \pm 3.31$ & $-0.74 \pm 2.01$ & 97 & {$[-1.15 ;-0.34]$} & 0.001 \\
\hline $\begin{array}{l}\text { Icon } \\
\text { WSL / } \\
\text { AGS }\end{array}$ & $\Delta \mathrm{E}$ & $8.14 \pm 4.59$ & $5.59 \pm 2.82$ & $2.55 \pm 4.24$ & 57 & {$[1.43 ; 3.68]$} & $<0.001$ \\
\hline $\begin{array}{l}\text { Kontroll } \\
\text { WSL / } \\
\text { AGS }\end{array}$ & $\Delta \mathrm{E}$ & $7.9 \pm 4.51$ & $7.6 \pm 4.51$ & $0.29 \pm 2.38$ & 57 & {$[-0.34 ; 0.93]$} & 0.35 \\
\hline
\end{tabular}

Tabelle IV: Der Intra-Gruppen-Vergleich der verschiedenen Werte von t0 bis t5 für beide Gruppen. 
Die Helligkeit der AGS hat sich durch die Icon-Behandlung nicht signifikant geändert, während sich die der WSL deutlich verändert hat. Es gab keine signifikanten Veränderungen der $L^{*}$-Werte in der Kontrollgruppe. Der $a^{*}$-Wert der WSL ändert sich durch die Behandlung mit Icon signifikant mit einem Anstieg im Sinne einer Verschiebung auf der Rot-Grün-Achse in Richtung ,rot'. Die $a^{*}$-Werte der AGS und der Kontrollgruppe veränderten sich weder mit noch ohne Icon-Behandlung.

Der $b^{*}$-Wert der WSL stieg durch die Icon-Behandlung signifikant von 17.75 auf 20.97, veränderte sich aber auch in der Kontrollgruppe von 16.27 auf 17.88. Bei dem b*-Wert der AGS ist es ähnlich: Er stieg signifikant in der Icon-Gruppe von 20.57 auf 22.19 und auch in der Kontrollgruppe von 20.68 auf 21.42 (siehe Tabelle IV, Seite 39). Es ist jedoch fraglich, ob die durchschnittliche Differenz von -0,74 von klinischer Relevanz ist.

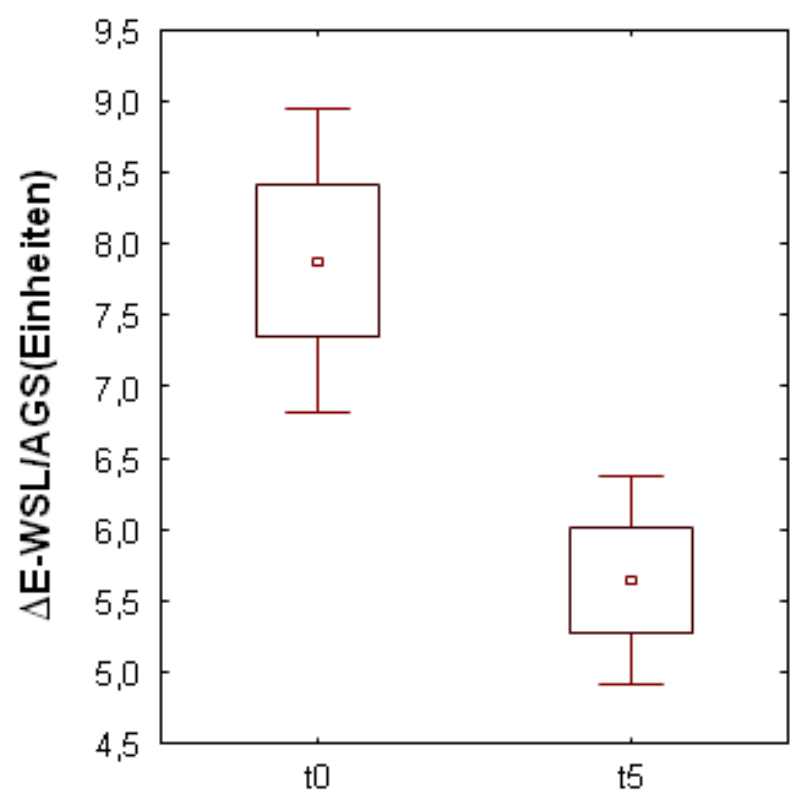

icon

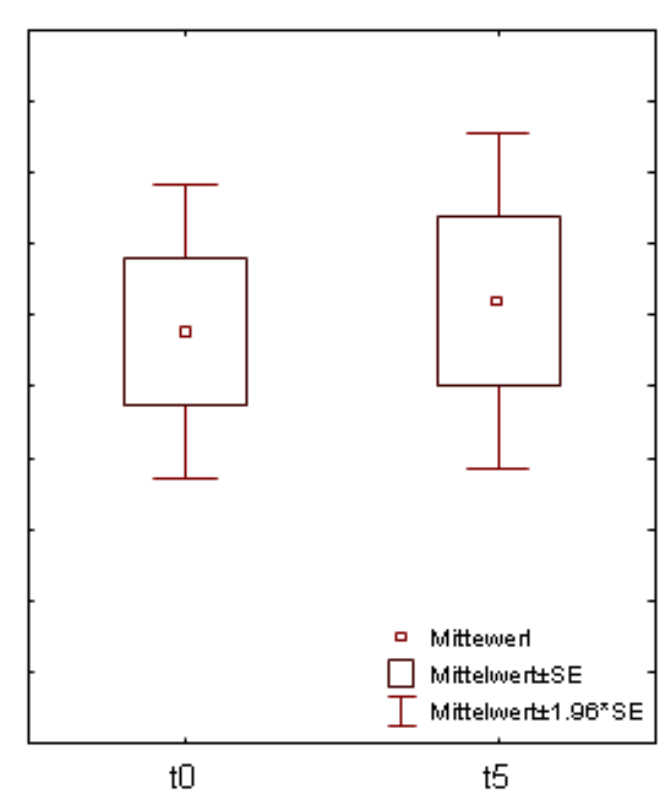

Kontrollgruppe

Zeit

Abbildung 12: Ausgehend von den Baseline-Werten (t0) waren die Farb- und Helligkeitswerte ( $\Delta \mathrm{E}-$ Werte) der WSL im Vergleich zu den Schmelzarealen nach sechs Monaten (t5) signifikant reduziert, während es bei den Kontrollzähnen keine signifikanten Änderungen gab. Die Null-Hypothese, dass es keine signifikanten Farb- und Helligkeitsveränderungen (CIE-L*a*b*, $\Delta E$-Werte) zwischen WSL und AGS sechs Monate nach Infiltration gibt, wurde zurückgewiesen.

Die $\triangle \mathrm{E}$ Unterschiede von WSL/AGS an den Icon-Zähnen erreichten den Schwellenwert der klinischen Sichtbarkeit, der mit dem bloßen Auge bei 3,7 $\Delta \mathrm{E}$-Einheiten liegt (Johnston und Kao 1989). 


\subsection{Infiltrierte Zähne}

Nach der Infiltration (t1) war eine signifikante Minderung der Farb- und Helligkeitsunterschiede zwischen WSL und AGS im Vergleich zu Baseline (t0) festzustellen. Das heißt, dass sich die Farbe der WSL an die Farbe des umliegenden Schmelzes optisch angeglichen hat. Diese Ergebnisse beziehen sich auf die $\Delta \mathrm{E}$-Werte in der Icon Gruppe. Der besagte Unterschied ändert sich nicht signifikant über die sechs Monate (t1 bis t5), was auf einen dauerhaften Camouflage-Effekt der Infiltration hindeutet. Die Abbildungen 10 (Seite 36), 12 (Seite 40) und die Tabelle V stellen die Entwicklung der zusammengefassten Farbund Lichtunterschiede in $\Delta \mathrm{E}-$ Werten dar. Die Null-Hypothese, dass es nach der Infiltration keine signifikanten Farb- und Helligkeitsveränderungen (durch CIE-L*a*b* $\Delta E-$ Werte) zwischen WSL (t0: 7.88 zu t5: 5.65; siehe Tabelle V) und den umliegenden Schmelzgebieten (t0: 7.38 zu t5: 7.59) im Vergleich zu der nicht behandelten Kontrollgruppe gibt, konnte zurückgewiesen werden.

Entwicklung der Farb- und Helligkeitsunterschiede zwischen WSL und AGS

\begin{tabular}{|l|l|l|l|l|l|l|}
\hline Gruppe & Zeit & $\Delta$ E-Mittelwert & $\mathrm{n}$ & $\mathrm{SD}$ & Minimum & Maximum \\
\hline Icon & $\mathrm{t} 0$ & 7.87 & 67 & 4.43 & 2.52 & 30.43 \\
\hline Icon & $\mathrm{t} 1$ & 6.09 & 67 & 3.24 & 0.27 & 14.71 \\
\hline Icon & $\mathrm{t} 2$ & 5.85 & 59 & 2.91 & 1.20 & 16.08 \\
\hline Icon & $\mathrm{t} 3$ & 5.66 & 65 & 2.58 & 1.07 & 14.63 \\
\hline Icon & $\mathrm{t} 4$ & 5.73 & 56 & 2.76 & 0.97 & 13.30 \\
\hline Icon & $\mathrm{t} 5$ & 5.64 & 58 & 2.83 & 0.80 & 13.11 \\
\hline Kontrollgruppe & $\mathrm{t} 0$ & 7.38 & 68 & 4.34 & 0 & 17.20 \\
\hline Kontrollgruppe & $\mathrm{t} 1$ & 8.61 & 65 & 5.01 & 0 & 21.89 \\
\hline Kontrollgruppe & $\mathrm{t} 2$ & 6.64 & 59 & 4.20 & 0 & 17.07 \\
\hline Kontrollgruppe & $\mathrm{t} 3$ & 7.01 & 68 & 4.39 & 0 & 18.05 \\
\hline Kontrollgruppe & $\mathrm{t} 4$ & 7.26 & 56 & 4.25 & 0.48 & 17.03 \\
\hline Kontrollgruppe & $\mathrm{t} 5$ & 7.59 & 57 & 4.51 & 0.48 & 20.23 \\
\hline
\end{tabular}

Tabelle V: Deskriptive Statistik 
Die Tabelle IV (Seite 39) und die Abbildungen 13a-f und 14a-f geben detaillierte Informationen über Farbe und Helligkeitsentwicklung der WSL und der AGS von t0 bis t5 in der Behandlungs- und der Kontrollgruppe. In den Abbildungen 13a-f zeigen die Grafiken die Veränderungen in den Gruppen (Kontrollgruppe oder Icon), während in den Abbildungen 14a-f die Veränderungen in den WSL und den AGS direkt gegenübergestellt werden.

Die Grafiken 13 und 14 sind im Folgenden unter den jeweiligen Kapiteln der zugehörigen CIE-L*a*b*-Werte eingeordnet ( $L^{*}$-Wert: 13a,b/14a,b Seiten 43/44; $a^{*}-$ Wert:13c,d/14c,d Seiten 45/46; b*-Wert: 13e,f/14e,f Seiten 47/48).

Dort werden die Entwicklungen der separaten Farb- und Helligkeitswerte in beiden Gruppen dargestellt.

Abbildungen 13a-f: Intra-Gruppen-Vergleiche zwischen WSL und AGS ausgehend vom Zeitpunkt t0 (Baseline) bis t5 (nach sechs Monaten) jeweils für die Behandlungs- und die Kontrollgruppe.

Abbildungen 14a-f: Inter-Gruppen-Vergleiche zwischen Icon-Behandlung und Kontrollgruppe ausgehend vom Zeitpunkt to (Baseline) bis t5 (nach sechs Monaten) jeweils für die WSL und die AGS. 


\subsection{1. $\quad L^{*}$-Wert (Helligkeit)}

Es gab eine signifikante Abnahme der Helligkeit der WSL durch die Infiltration, aber keine signifikante Veränderung der Helligkeit über sechs Monate in der Schmelzregion der infiltrierten Zähne.

Es wurden auch keine signifikanten Helligkeitsveränderungen in der Kontrollgruppe verzeichnet (weder in den WSL, noch in den AGS); (Abbildung 13a, b).

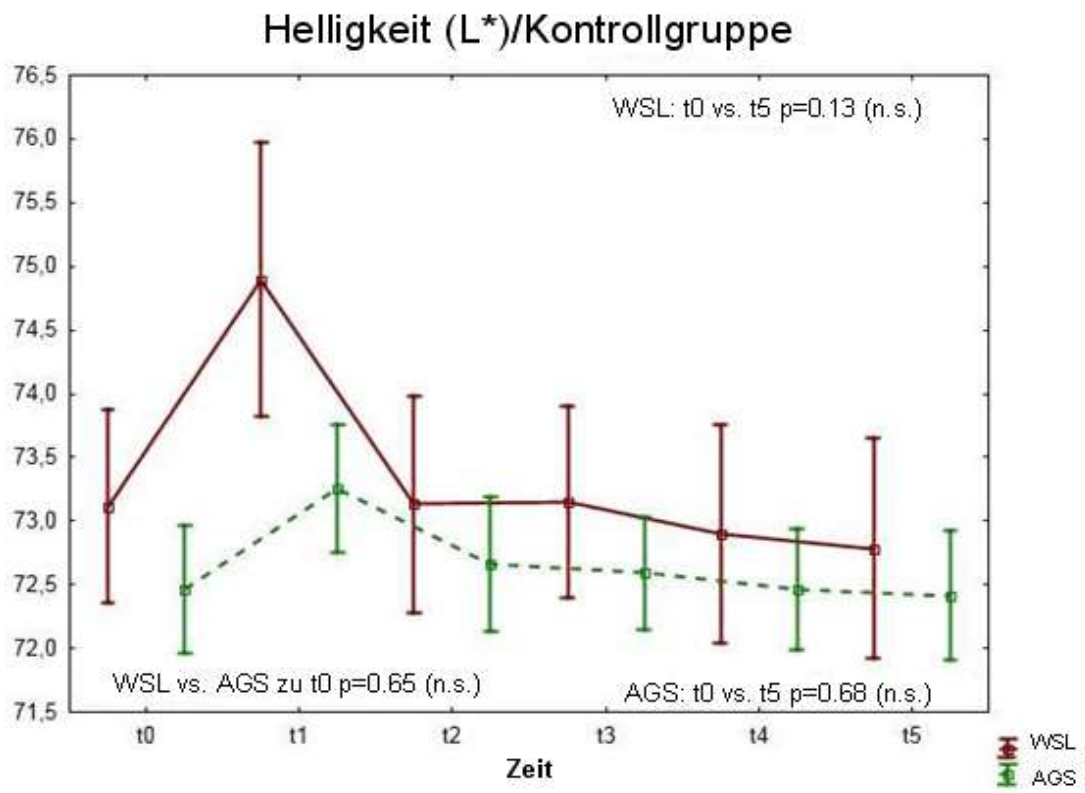

Abbildung 13a

Helligkeit $\left(L^{*}\right) / / c o n$

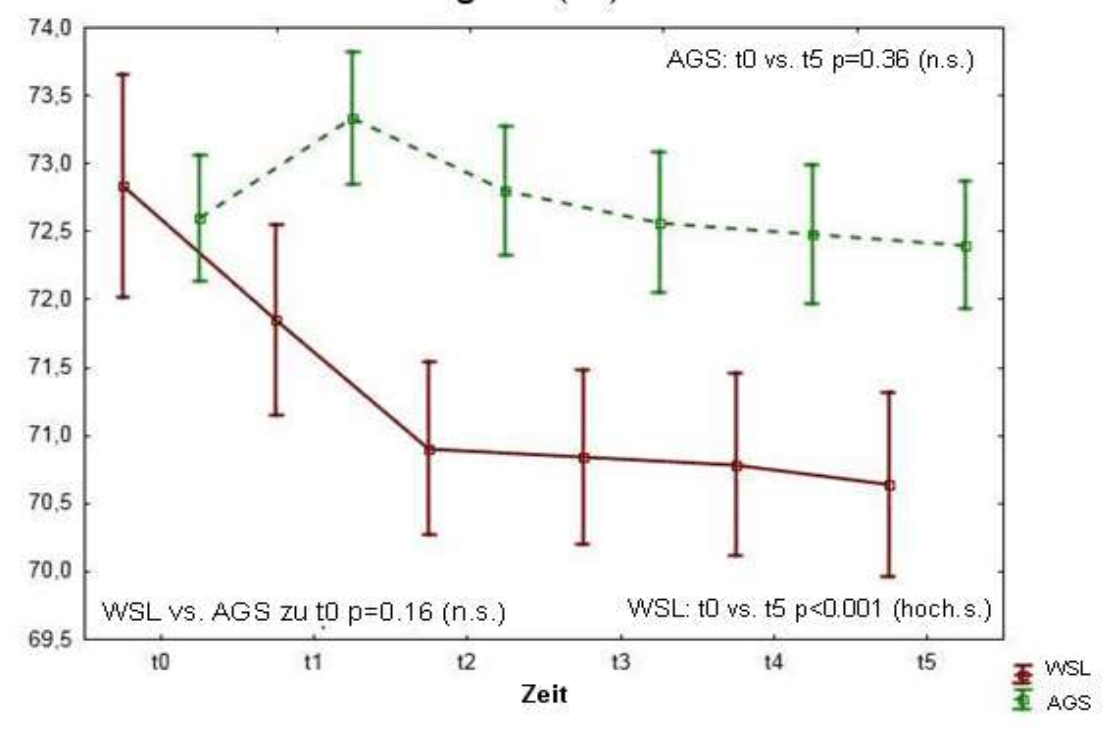

Abbildung 13b 
Ergebnisse

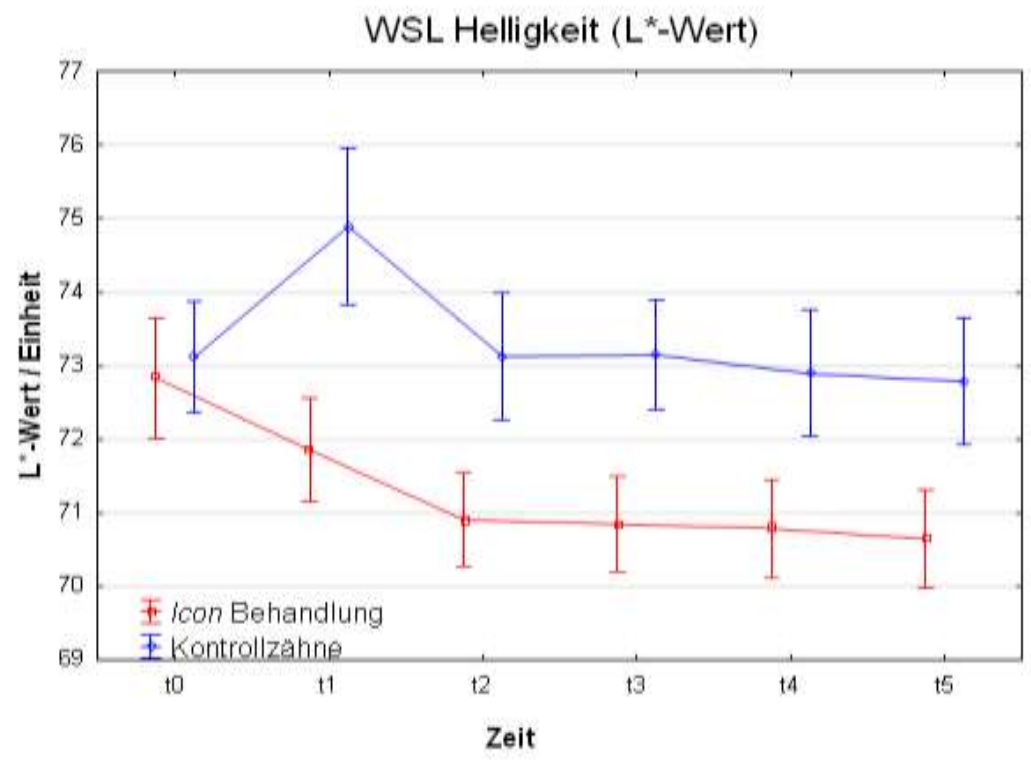

Abbildung 14a

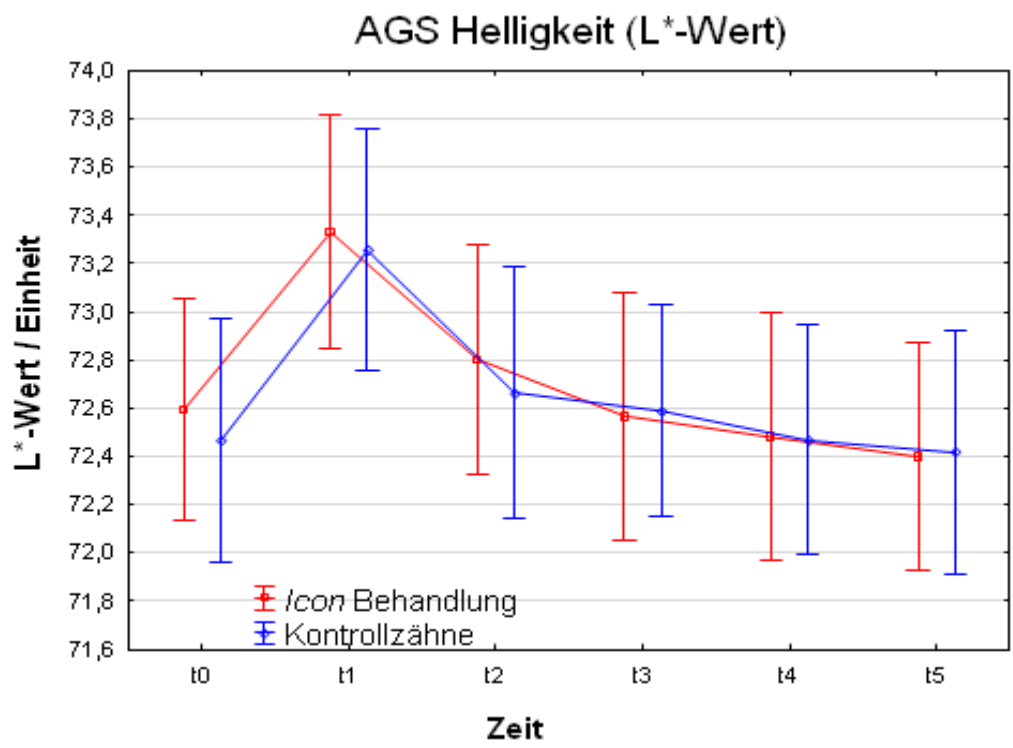

Abbildung 14b 


\subsection{2. $\quad a^{*}$-Wert (Rot-Grün-Achse)}

Die Farbe der WSL veränderte sich signifikant nach der Infiltration mit einem hohen signifikanten Anstieg im $a^{*}-$ Wert. Das bedeutet eine Veränderung auf der Rot-Grün-Achse zum ,Rot'-Pol. Währenddessen war weder in der Icon-AGS-Fläche noch in den WSL oder den AGS der Kontrollzähne eine signifikante Veränderung festzustellen. (Abbildungen 13c, d und $14 \mathrm{c}, \mathrm{d})$.

Rot-Grün-Achsen-

Veränderungen $\left(a^{*}\right) /$ Kontrollgruppe

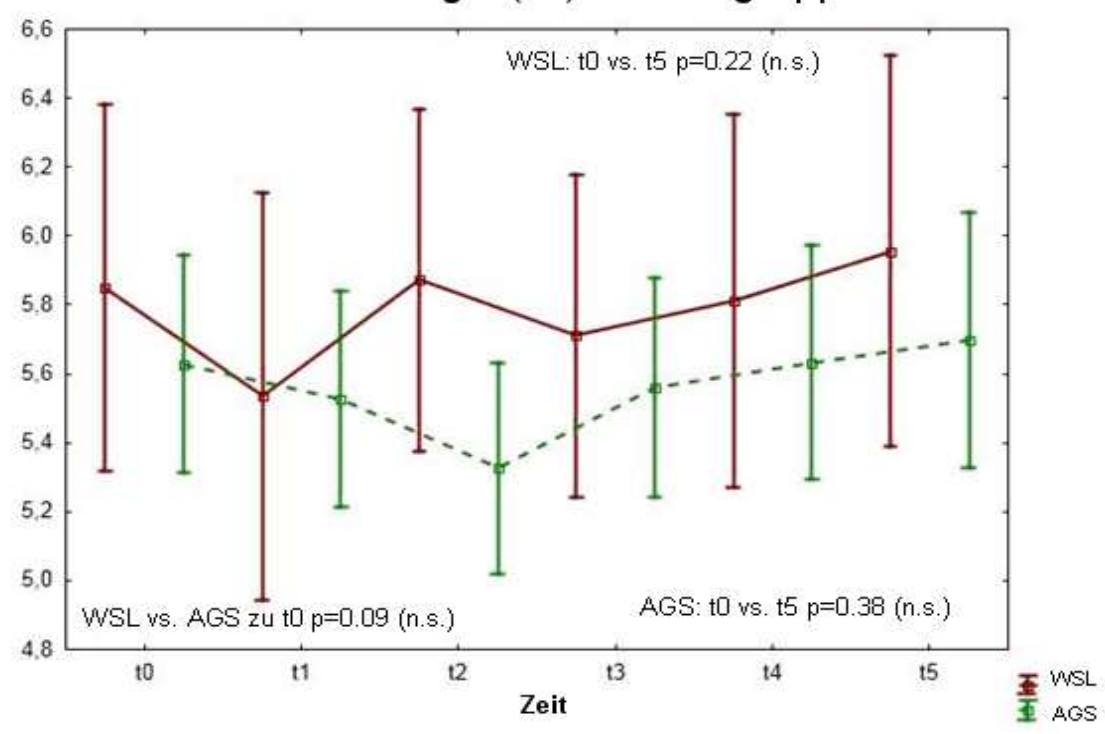

Abbildung $13 \mathrm{c}$

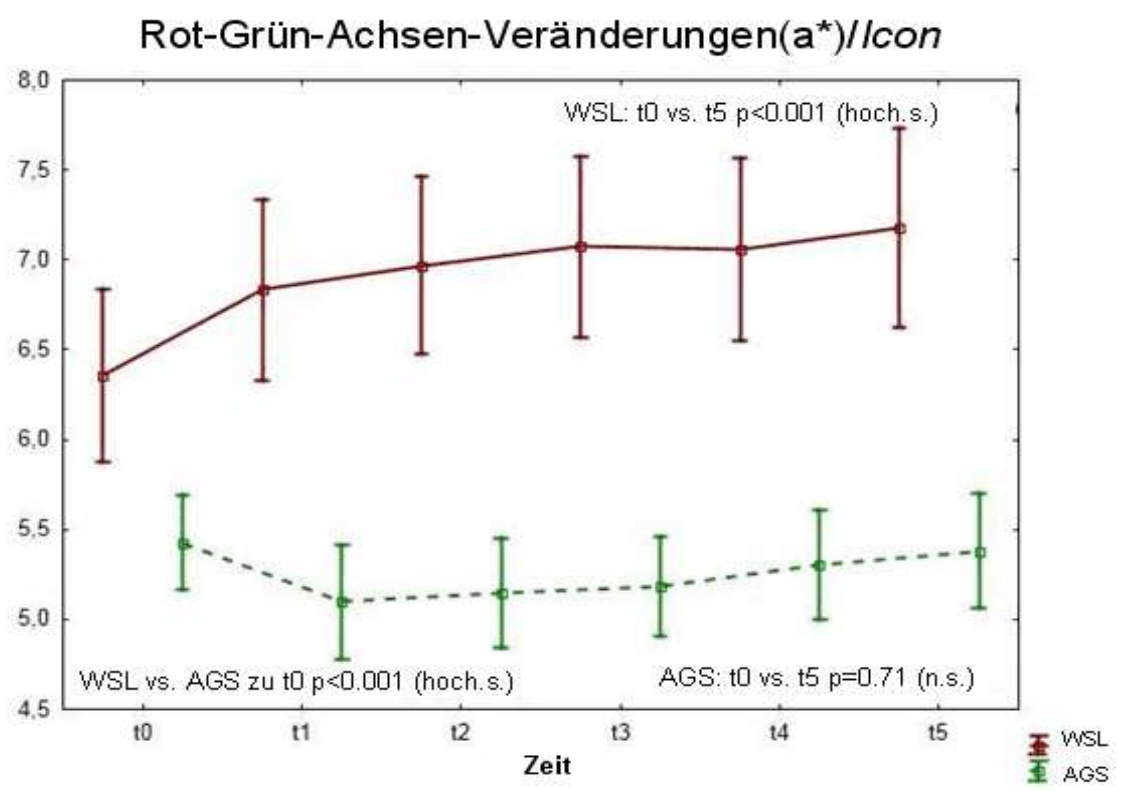

Abbildung 13d 


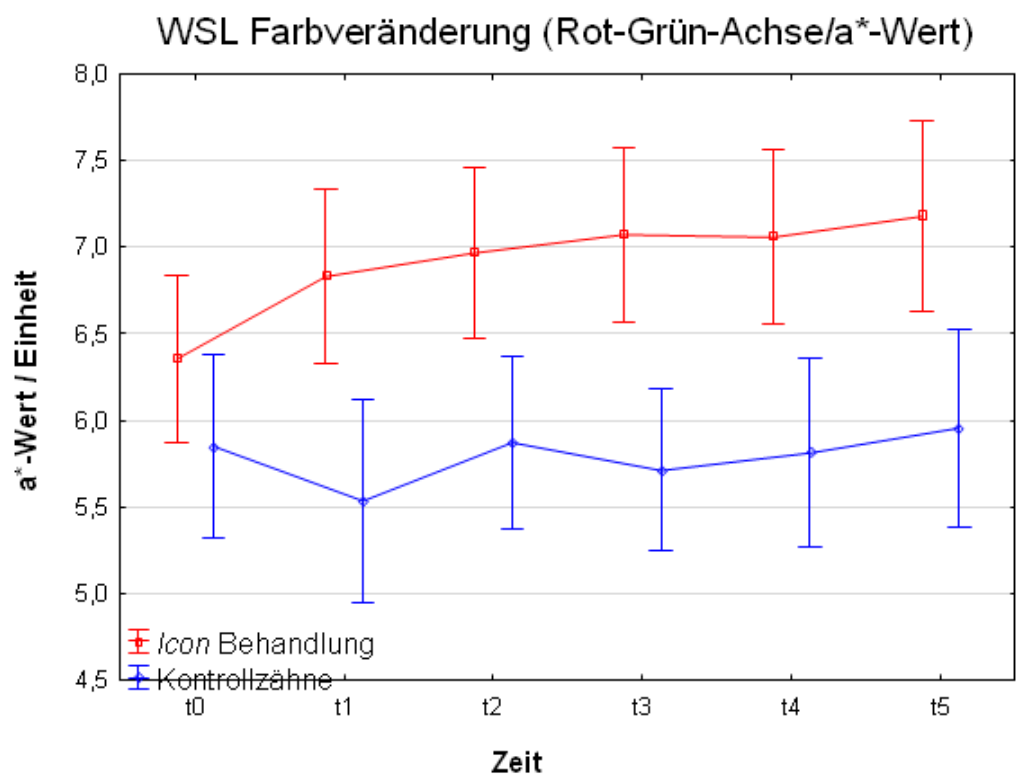

Abbildung $14 \mathrm{c}$

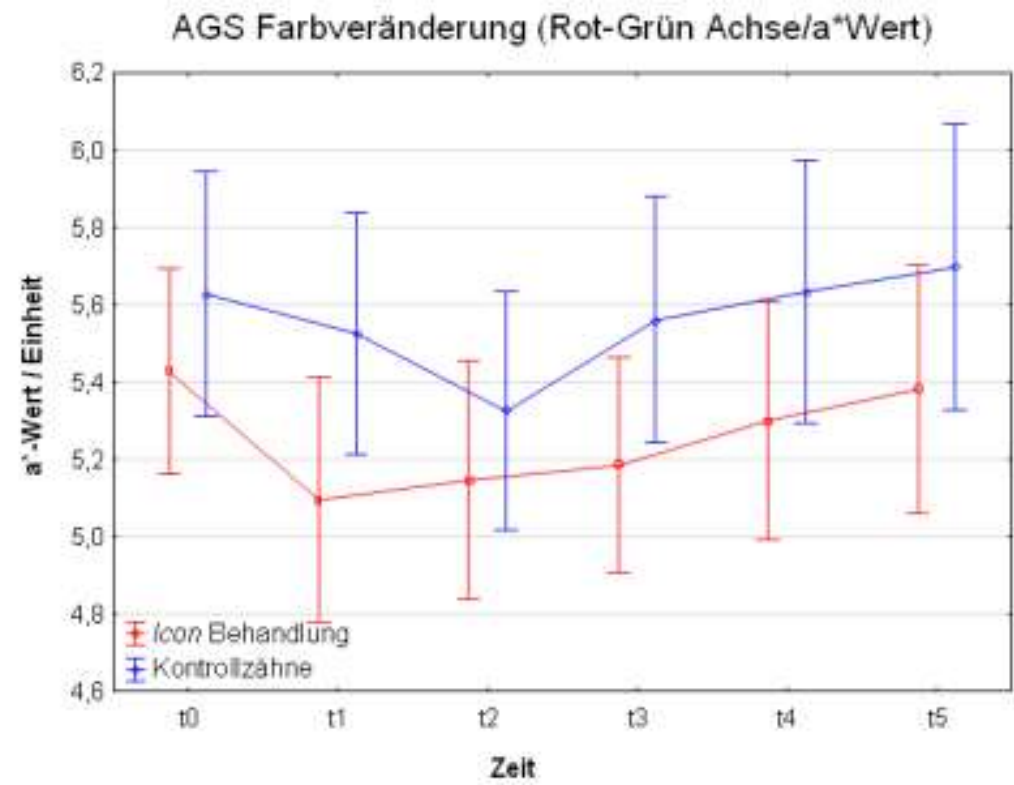

Abbildung $14 \mathrm{~d}$

Abbildung 14 C und D: Der a*-Wert steigt durch die Icon-Behandlung an (Abbildung 14c), während im AGS keine signifikante Veränderung stattfindet (Abbildung 14d). 


\subsection{3. $\quad b^{*}$-Wert (Blau-Gelb-Achse)}

Die $b^{*}$-Werte der WSL und AGS veränderten sich durch die Icon-Behandlung hoch signifikant von Baseline bis t5 (WSL stieg von 17.7 auf 20.9, AGS stieg von 20.5 auf 22.2). Das bedeutet eine Veränderung auf der Blau-Gelb-Achse in Richtung des ,Gelb'-Pols. Hier gab es auch eine hoch signifikante Veränderung in der Kontrollgruppe. (Anstieg von $b^{*}$ bei den WSL von 16.2 auf 17.8, bei den AGS von 20.7 auf 21.4) (Abbildung 13 e, f).

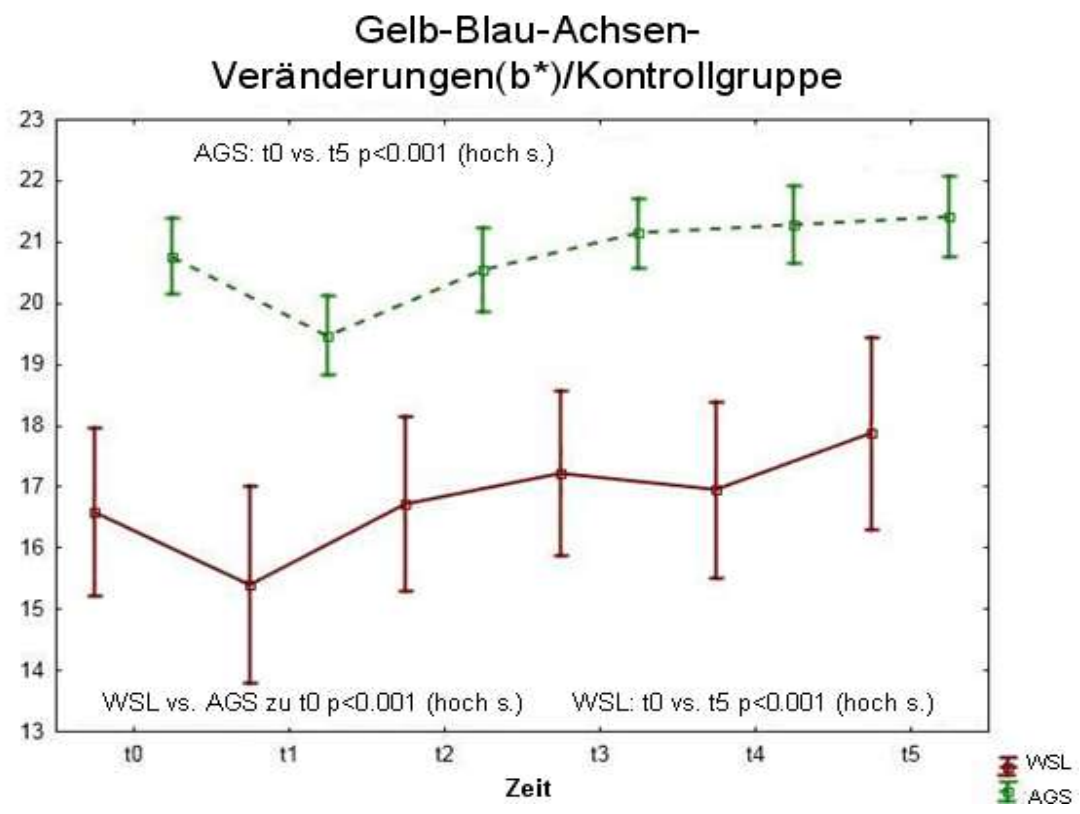

Abbildung 13e

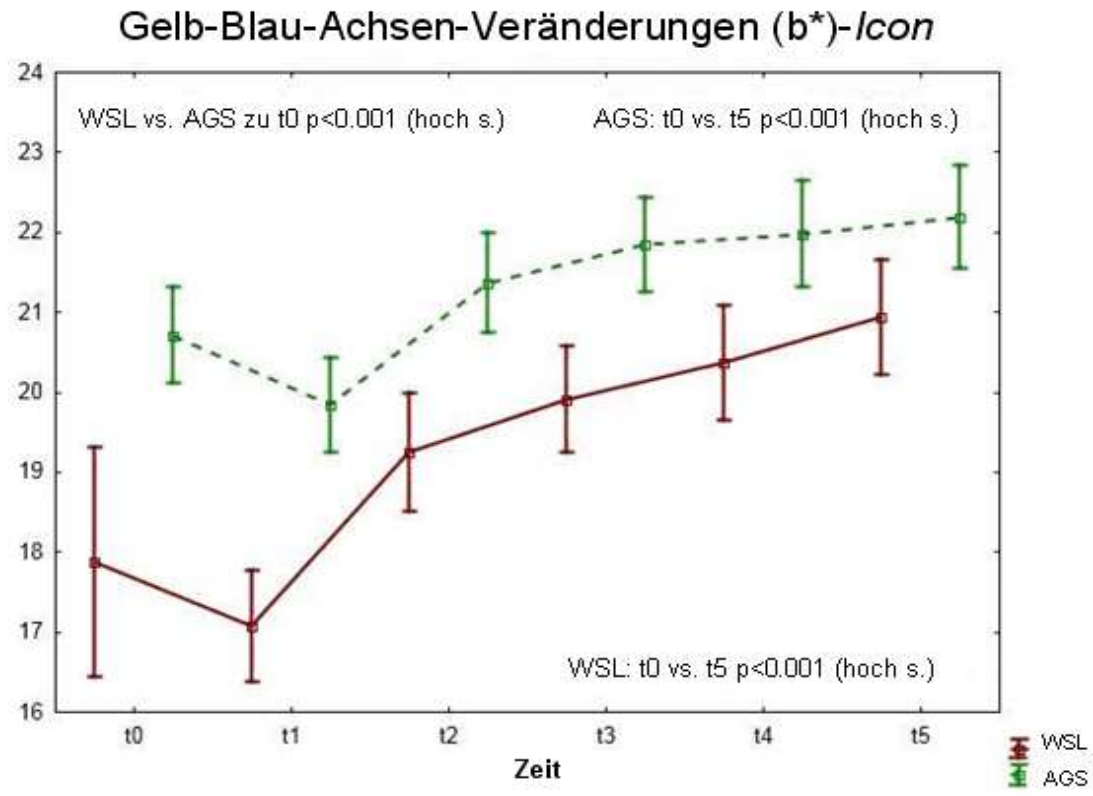

Abbildung 13f 


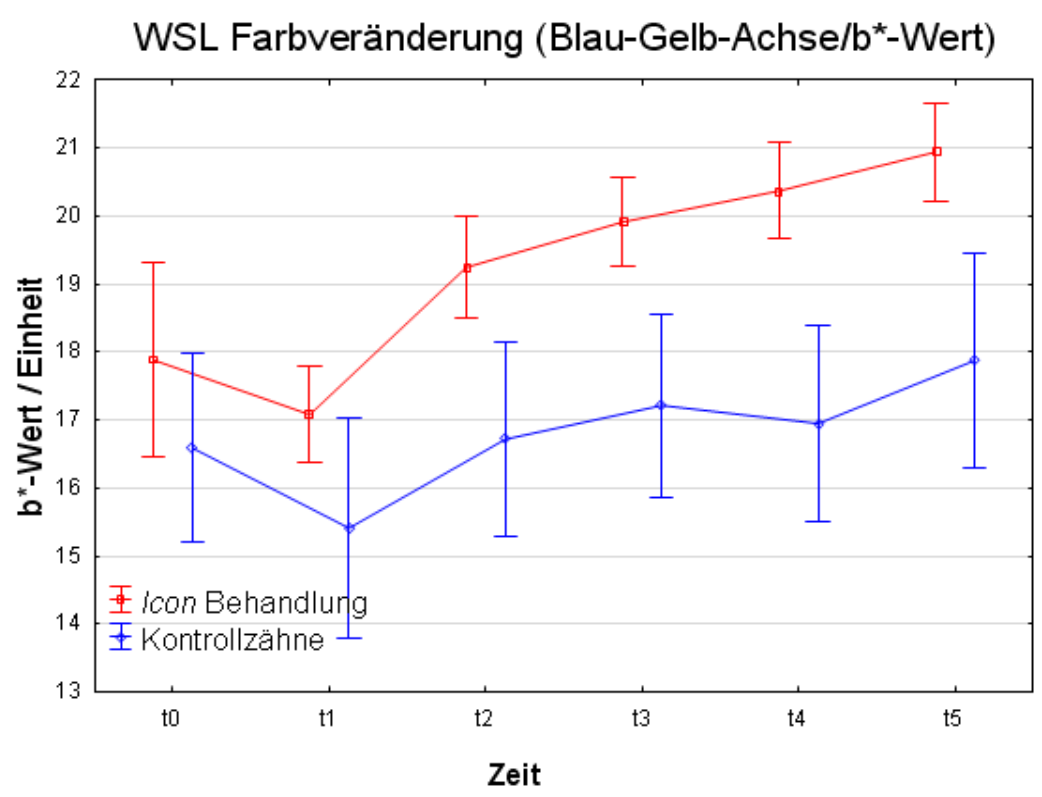

Abbildung $14 \mathrm{e}$

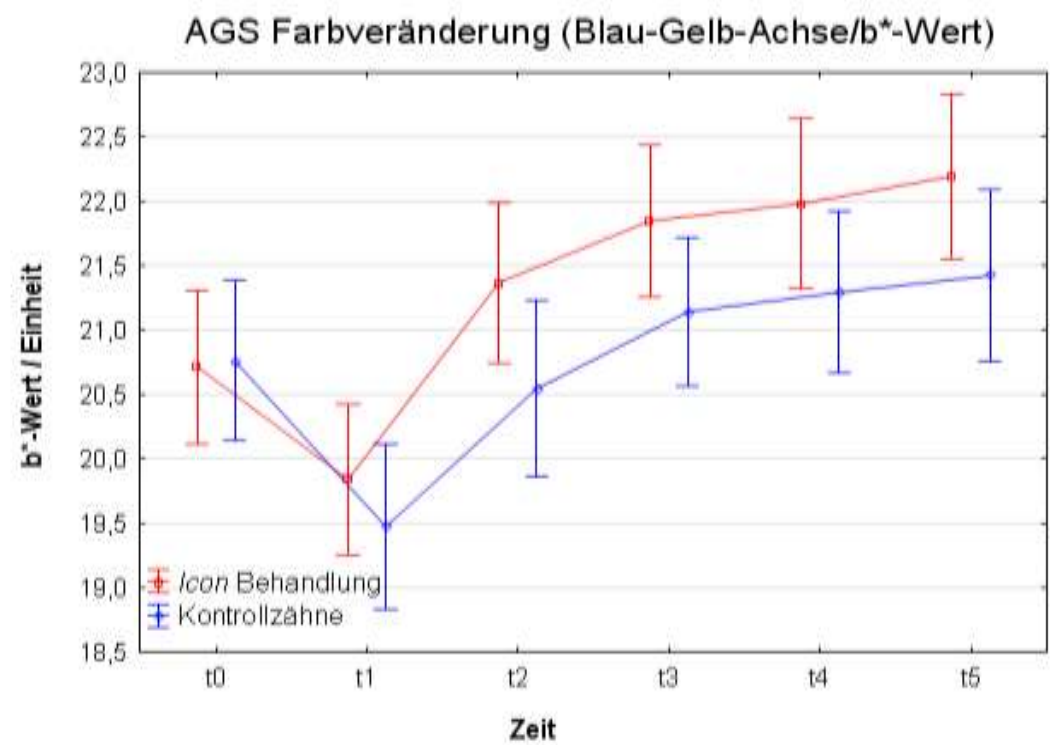

Abbildung $14 \mathrm{f}$

\subsection{Zusammenfassung 6-Monats-Ergebnisse}

Beide Parameter ,Behandlung' und ,Zeitablauf' zeigen einen hoch signifikanten Einfluss auf die $\Delta \mathrm{E}$-Werte. Es gab einen optischen Angleich der Farbe der WSL an die der umgebenden Schmelzareale, der über sechs Monate ohne signifikante Veränderungen farbstabil blieb. In der Gruppe der unbehandelten Zähne gab es in dem halben Jahr keine signifikanten Veränderungen im Vergleich zu Baseline. 


\subsection{Nachuntersuchung der infiltrierten Zähne nach 12 Monaten}

Als Neben-Experiment zu der vorliegenden Studie wurden die infiltrierten Zähne zusätzlich noch einmal nach 12 Monaten gemessen (t6). Allerdings wurde zu Beginn der Studie mit den Patienten vereinbart, dass die Zähne des Kontrollquadranten bei Interesse nach der t5Messung (nach einem halben Jahr) ebenfalls mit Icon behandelt werden können. Alle Patienten, die an der Studie teilnahmen, haben sich nach einem halben Jahr entschlossen, die Zähne der Kontrollgruppe infiltrieren zu lassen. Daher konnten zum Zeitpunkt t6 nur noch die zur Baseline (t0) Icon-Zähne gemessen werden. Die Messung der Kontrollquadranten entfiel, die Analyse der t6-Messungen erfolgte Baseline-kontrolliert und im Vergleich zu den t5-Messungen.

Von 20 kontaktierten Patienten konnten neun Patienten mit insgesamt $n=49$ Zähnen (96 Flächen) im t6-Recall gemessen werden. Alle Vergleiche wurden mittels verbundenem t-Test zum Fehlerniveau alpha=5\% durchgeführt. Dabei wurden, wie aus Tabelle VI (Seite 50) ersichtlich Vergleiche zwischen t0, t5 und t6 durchgeführt. Die $\Delta \mathrm{E}$-Werte beziehen sich auf die Icon-Quadranten.

Der Unterschied von t0 zu t5 war hoch signifikant ( $p<0,001)$. Die Differenz $\Delta E$ (WSL/AGS) zum Zeitpunkt t6 betrug 5.55 Einheiten (SD 2.6), zu t5 6.33 Einheiten (SD 3.81) und $\Delta \mathrm{E}$ zu t0 8.15 Einheiten (SD 3.74). Daraus lässt sich ermitteln, dass auch der Unterschied t0-t6 hoch signifikant war $(p<0.001)$ (siehe Tabelle VII, Seite 51).

Obwohl sich die $\Delta$ E-Differenz (WSL/AGS) im Vergleich zur Baseline und zu t5 bei t6 noch weiter verringert hat, ist dieser Unterschied t5-t6 nicht signifikant. Das bedeutet, dass die infiltrierten WSL durch die noch stärkere Absenkung der summierten Farb- und Helligkeitsunterschiede zwischen infiltriertem und gesundem Schmelz theoretisch noch etwas weniger sichtbar sein müssten, was aber wahrscheinlich im klinischen Setting kaum wahrnehmbar ist (Johnston und Kao 1989). 
Tabelle VI: Deskriptive Statistik: CIE-L*-, $a^{*}-$, und $b^{*}$-Werte der infiltrierten WSL und den AGS sowie die WSL/AGS-Unterschiede zu den verschiedenen Zeitpunkten.

\begin{tabular}{|c|c|c|c|c|c|c|c|c|}
\hline \multicolumn{2}{|c|}{$\begin{array}{l}\text { Vergleich der } \\
\text { Zeitpunkte ts } \\
\text { Zeitpunkt 1/ Zeitpunkt2 }\end{array}$} & Bereich & $\begin{array}{l}\text { Para- } \\
\text { meter }\end{array}$ & $\begin{array}{c}\text { Mittelwert } \pm S D \\
\text { Zeitpunkt } 1\end{array}$ & $\begin{array}{c}\text { Mittelwert } \pm S D \\
\text { Zeitpunkt } 2\end{array}$ & Diff. & $\mathrm{p}$ & Cl 95\% \\
\hline to & t5 & WSL & L & $72.55 \pm 3.45$ & $70.88 \pm 3.49$ & 1.68 & 0.008 & {$[0.47 ; 2.88]$} \\
\hline to & $t 6$ & WSL & L & $72.55 \pm 3.45$ & $70.37 \pm 3.16$ & 2.19 & $<0.001$ & {$[0.99 ; 3.38]$} \\
\hline to & t5 & AGS & L & $72.8 \pm 2.92$ & $72.74 \pm 2.34$ & 0.05 & 0.85 & {$[-0.51 ; 0.62]$} \\
\hline to & t6 & AGS & L & $72.8 \pm 2.92$ & $72.50 \pm 2.21$ & 0.30 & 0.42 & {$[-0.43 ; 1.03]$} \\
\hline to & $t 5$ & $\begin{array}{l}\text { WSL- } \\
\text { AGS }\end{array}$ & Diff $L$ & $-0.25 \pm 2.73$ & $-1.87 \pm 3.34$ & 1.62 & 0.003 & {$[0.60 ; 2.65]$} \\
\hline to & $t 6$ & $\begin{array}{l}\text { WSL- } \\
\text { AGS }\end{array}$ & Diff $L$ & $-0.25 \pm 2.73$ & $-2.13 \pm 2.94$ & 1.89 & $<0.001$ & {$[0.97 ; 2.80]$} \\
\hline to & $t 5$ & WSL & a & $6.54 \pm 2.21$ & $7.56 \pm 2.47$ & -1.02 & $<0.001$ & {$[-1.50 ;-0.54]$} \\
\hline to & t6 & WSL & a & $6.54 \pm 2.21$ & $8.00 \pm 2.67$ & -1.45 & $<0.001$ & {$[-2.20 ;-0.70]$} \\
\hline to & t5 & AGS & a & $5.39 \pm 1.52$ & $5.28 \pm 1.66$ & 0.11 & 0.35 & {$[-0.13 ; 0.36]$} \\
\hline to & t6 & AGS & $a$ & $5.39 \pm 1.52$ & $5.38 \pm 1.61$ & 0.01 & 0.95 & {$[-0.33 ; 0.35]$} \\
\hline to & $t 5$ & $\begin{array}{l}\text { WSL- } \\
\text { AGS }\end{array}$ & Diff a & $1.15 \pm 2.11$ & $2.28 \pm 2.20$ & -1.13 & $<0.001$ & {$[-1.57 ;-0.70]$} \\
\hline to & t6 & $\begin{array}{l}\text { WSL- } \\
\text { AGS }\end{array}$ & Diff a & $1.15 \pm 2.11$ & $2.61 \pm 2.30$ & -1.46 & $<0.001$ & {$[-2.03 ;-0.89]$} \\
\hline to & $t 5$ & WSL & $b$ & $16.24 \pm 5.31$ & $20.48 \pm 4.68$ & -4.24 & $<0.001$ & {$[-5.94 ;-2.54]$} \\
\hline to & t6 & WSL & b & $16.24 \pm 5.31$ & $21.88 \pm 3.47$ & -5.64 & $<0.001$ & {$[-7.51 ;-3.76]$} \\
\hline to & $t 5$ & AGS & $b$ & $20.72 \pm 3.57$ & $22.56 \pm 3.40$ & -1.83 & $<0.001$ & {$[-2.39 ;-1.28]$} \\
\hline to & t6 & AGS & $b$ & $20.72 \pm 3.57$ & $22.71 \pm 3.37$ & -1.99 & $<0.001$ & {$[-2.69 ;-1.29]$} \\
\hline to & $t 5$ & $\begin{array}{l}\text { WSL- } \\
\text { AGS }\end{array}$ & Diff $b$ & $-4.48 \pm 6.93$ & $-2.07 \pm 5.12$ & -2.40 & 0.001 & {$[-3.81 ;-0.99]$} \\
\hline to & $t 6$ & $\begin{array}{l}\text { WSL- } \\
\text { AGS }\end{array}$ & Diff $b$ & $-4.48 \pm 6.93$ & $-0.83 \pm 3.46$ & -3.65 & $<0.001$ & {$[-5.44 ;-1.85]$} \\
\hline
\end{tabular}


Tabelle VII: Entwicklung der summierten Farb- und Helligkeitsunterschiede zwischen WSL und dem angrenzenden gesunden Schmelz (AGS) sowie WSL/AGS Unterschiede in den $\Delta \mathrm{E}_{\text {WSL/AGS }}$-Werten zu den verschiedenen Zeitpunkten. Im Vergleich zu t0 zeigen die P-Werte eine hohe Signifikanz. Die Veränderung zwischen t5 und t6 hingegen ist nicht signifikant.

\begin{tabular}{|c|c|c|c|c|c|c|c|}
\hline \multicolumn{2}{|c|}{$\begin{array}{l}\text { Vergleich der } \\
\text { Zeitpunkte } \\
\text { Zeitpunkt 1/ Zeitpunkt2 }\end{array}$} & Parameter & $\begin{array}{c}\text { Mittelwert土SD } \\
\text { Zeitpunkt } 1\end{array}$ & $\begin{array}{c}\text { Mittelwert+SD } \\
\text { Zeitpunkt } 2\end{array}$ & Diff. & $P$ & Cl 95\% \\
\hline to & t5 & $\Delta \mathrm{E}_{\mathrm{WSL} / \mathrm{AGS}}$ & $8.15 \pm 3.74$ & $6.33 \pm 3.81$ & 1.82 & $<0.001$ & {$[0.84 ; 2.79]$} \\
\hline to & t6 & $\Delta \mathrm{E}_{\text {WSL/AGS }}$ & $8.15 \pm 3.74$ & $5.55 \pm 2.61$ & 2.60 & $<0.001$ & {$[1.45 ; 3.76]$} \\
\hline t5 & t6 & $\Delta \mathrm{E}_{\text {WSL/AGS }}$ & $6.33 \pm 3.81$ & $5.55 \pm 2.61$ & 0.79 & 0.08 & {$[-0.10 ; 1.67]$} \\
\hline
\end{tabular}

Tabelle VIII: Paarweise t-Tests zeigen hoch signifikante Unterschiede in den summierten Farb- und Helligkeitswerten von entweder WSL- oder AGS-Bereichen ( $\Delta \mathrm{E}_{\mathrm{WSL}} ; \Delta \mathrm{E}_{\mathrm{AGS}}$ ) zwischen Baseline und nach dem Verstreichen von sechs (t5) oder zwölf (t6) Monaten.

\begin{tabular}{|l|c|c|c|c|c|c|c|}
\hline Bereich & $\begin{array}{c}\text { Vergleich der } \\
\text { Zeitpunkte }\end{array}$ & $\begin{array}{c}\text { Mittelwert } \pm \text { SD } \\
\text { Bereich 1 }\end{array}$ & $\begin{array}{c}\text { Mittelwert } \pm \text { SD } \\
\text { Bereich 2 }\end{array}$ & Diff. & P & Cl 95\% \\
\hline WSL & AGS & t0-t5 & $7.54 \pm 4.46$ & $2.91 \pm 1.77$ & 4.63 & $<0.001$ & $\begin{array}{c}{[3.54 ;} \\
5.72]\end{array}$ \\
\hline WSL & AGS & t0-t6 & $8.83 \pm 5.17$ & $3.51 \pm 2.31$ & 5.32 & $<0.001$ & $\begin{array}{c}{[4.10 ;} \\
6.55]\end{array}$ \\
\hline WSL & AGS & t5-t6 & $2.69 \pm 5.66$ & $1.14 \pm 2.09$ & 1.56 & 0.01 & $\begin{array}{c}{[0.343 ;} \\
2.78]\end{array}$ \\
\hline
\end{tabular}




\section{Ergebnisse}

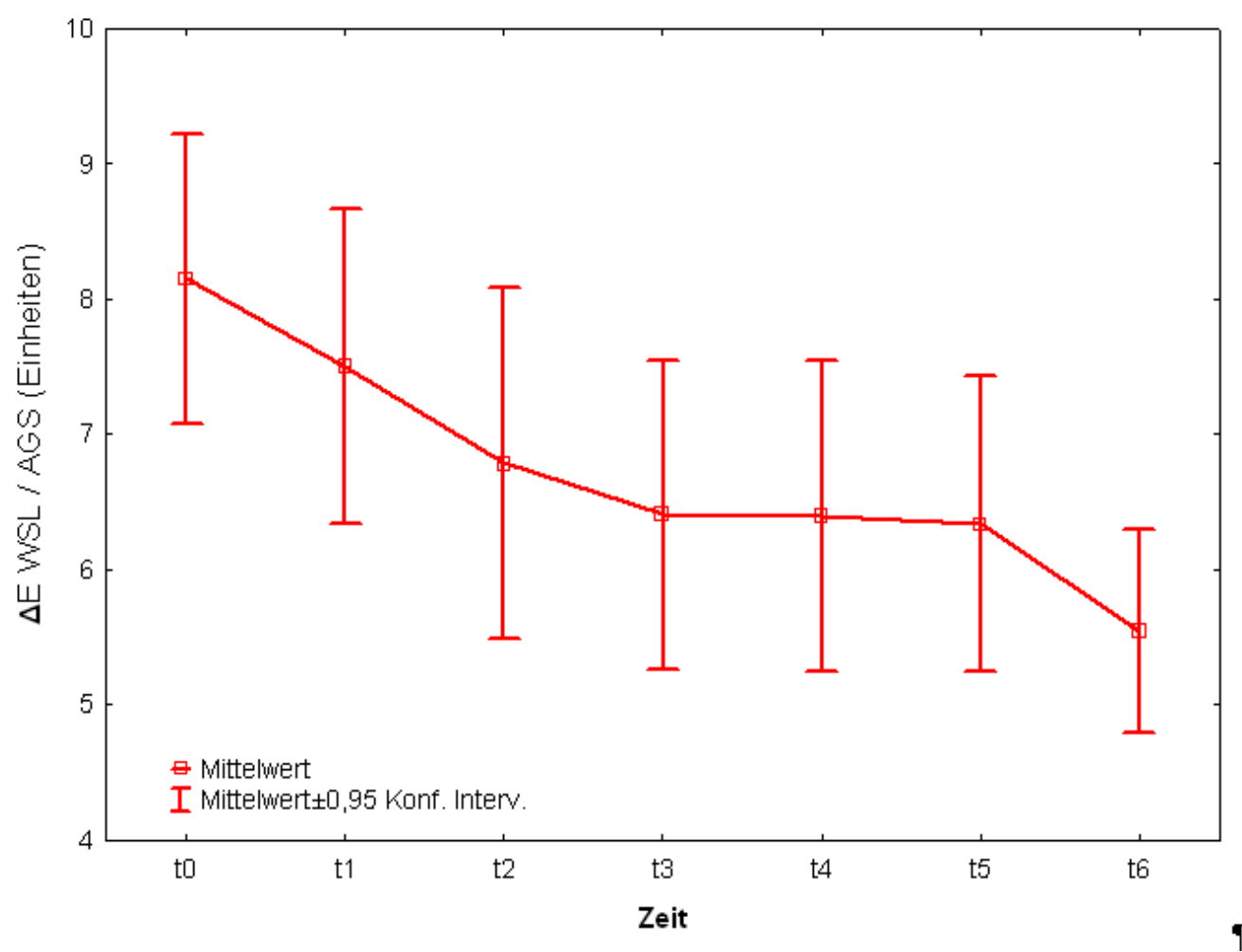

Abbildung 15: Es ist der stetige Abfall der $\triangle \mathrm{E}$-WSL/AGS-Werte zu sehen, der aber zwischen t5 und t6 keine Signifikanz aufweist. Die ursprünglichen Kontrollzähne waren zum Zeitpunkt des Nebenexperiments t6 bereits ebenfalls infiltriert und daher nicht mehr verfügbar. 


\section{Diskussion}

\subsection{Problemstellungen bei der WSL-Behandlung}

Die Behandlung von White-Spot-Läsionen soll dazu dienen, die Progression der Läsion aufzuhalten. Trotz zahlreicher Empfehlungen zur Remineralisation von WSL fehlen in der Literatur klare evidenzbasierte Richtlinien zur effektiven Behandlung (Kalha 2004). Allgemein wird, wie bei der WSL-Prophylaxe, zur Therapie der lokale Einsatz niedrig konzentrierter Fluoridspüllösungen empfohlen. Für diese Art der Remineralisation des erkrankten Schmelzes existiert eine „gewisse Evidenz“ („some evidence“, Kalha 2004)

Typischerweise kann innerhalb des ersten Jahres nach der Entbänderung eine geringe optische Verminderung von WSL beobachtet werden, was auf Remineralisationsvorgänge sowie Zahnbürstabrasion der oberflächlichen WSL-Schicht zurückzuführen ist (Backer Dirks 1966, Fejerskov et al. 2003, Holmen et al. 1987). Die in diesem Jahr entstehende pseudointakte Oberfläche der WSL weist eine erhöhte Härte im Vergleich zu ,frischen' WSL auf (Palamara et al. 1986, Al-Khateeb et al. 1998, Willmot 2004, Wiegand et al. 2007). Die Kombination aus Remineralisation und oberflächlicher Bürstabrasion resultiert also in einem Verlust der porösen Oberfläche.

Dennoch erfolgt keine vollständige restitutio ad integrum: Auch remineralisierte WSL bleiben dauerhaft sichtbar (Backer Dirks 1966, Fejerskov et al. 2003, Holmen et al. 1987) und bringen ästhetische Beeinträchtigungen der dentofazialen Ästhetik mit sich (Knösel et al. 2007).

Die Aufnahme von Lebensmittelfarbstoffen während der Remineralisierung in den Läsionen kann sogar zur Bildung sogenannter brown spot Läsionen führen (Hellwig et al. 2009), die das ästhetisches Problem verstärken können.

Abgesehen von der beeinträchtigten Ästhetik bedeuten WSL jedoch gleichzeitig eine Stufe initialer Schmelzkaries, die im Vergleich zur äußeren gesunden Schmelzschicht eine signifikant reduzierte Mineralisation zeigt (Palamara et al. 1986). Die Läsion kann sich, wenn die auslösende unzureichende Mundhygiene persistiert, bis zu einem Schweregrad fortsetzen, bei dem eine Restauration der dann entstandenen Karies notwendig wird (Featherstone 2000). Meyer-Lueckel und Paris betonten, dass die ursprüngliche Idee der Kariesinfiltration das Aufhalten der Progression der Läsion war, und zwar durch Verschließen der Mikroporositäten, die Säuren und aufgelösten Mineralien einen Diffusionsweg bieten (Meyer-Lueckel und Paris 2008). In verschiedenen Studien konnten Meyer-Lueckel und Paris belegen, dass infiltrierte Schmelzläsionen signifikant langsamer bis gar nicht mehr 
voranschreiten im Vergleich zu nicht behandelten Läsionen (Paris et al. 2010). Die Infiltrationsbehandlung hat sich somit als neuer Weg etabliert, WSL mit dem Ziel, die Kariesprogression zu inhibieren und gleichzeitig auch die Sichtbarkeit der WSL zu reduzieren, zu behandeln. Dieser Effekt ergibt sich daraus, dass sich die Opazität der WSL verringert und damit die Ästhetik an das Vorbild der Lichtbrechung gesunden Schmelzes angeglichen wird (Paris et al. 2010). Der Farbangleich ist darauf zurückzuführen, dass der Lichtbrechungsindex des Infiltranten ähnlich dem des Schmelzes ist (Paris et al. 2009) (siehe Punkt 6.2.1. Seite 53 und Abbildung 3, Seite 16).

Bei inaktiven labialen WSL kann dem ästhetischen Problem mehr Berücksichtigung geschenkt werden, als dem Risiko dieser WSL eine Karies auszubilden.

Bisher mögliche Aussagen zum Thema des Farbangleichs nach Infiltration bewegten sich auf niedrigem Evidenzniveau: So existierten lediglich In-vitro-Untersuchungen an infiltrierten Rinderschmelzproben mit sehr kurzen Beobachtungszeiträumen sowie vereinzelte Fallberichte (Gugnani et al. 2012, Gerard Kugel et al. 2009).

Während es bereits einige aussagekräftige Studien zur WSL-Infiltration gibt, die die Fähigkeit der Kariesinhibition thematisieren (Paris et al. 2009), fehlten bisher noch randomisierte, kontrollierte In-vivo-Langzeituntersuchungen zur Stabilität des Camouflage-Effekts. Diese werden aber benötigt, um eine Einschätzung der Haltbarkeit des Maskierungseffekts treffen und auch gegenüber potentiellen Patienten kommunizieren zu können.

\subsection{Patienten, Material und Methoden}

In der vorliegenden In-vivo-Studie wurden White-Spot-Läsionen (WSL) nach der Infiltration mit Icon über sechs Monate beobachtet. Hierbei wurden das Ausmaß und die Haltbarkeit der Farb- und Lichtveränderung zwischen den WSL und den daran angrenzenden Schmelzbereichen (AGS) nach Infiltration im Vergleich zu einer unbehandelten Kontrollgruppe bewertet. Die Auswertung wurde mit Hilfe von CIE-L*a* $\mathrm{b}^{*}$ - und $\triangle \mathrm{E}-$ Werten durchgeführt. Die Messungen fanden vor (t0), direkt (t1), eine Woche (t2), einen Monat (t3), drei Monate (t4) und sechs Monate (t5) nach der Infiltration statt. Die Null-Hypothese, dass keine signifikant unterschiedlichen $\Delta$-E-Werte (WSL vs. AGS) zwischen der behandelten Gruppe und der Kontrollgruppe im Verlauf von sechs Monaten auftreten, konnte anhand der Ergebnisse dieser Arbeit zurückgewiesen werden.

Die Genehmigung der Studie erfolgte durch die Ethikkommission der Universitätsmedizin Göttingen (siehe Anhang, Seite 85). Da die Studie in vivo durchgeführt wurde, bestand eine hohe Abhängigkeit von der Patientencompliance. Demineralisationen ungeschützten Schmelzes sind während der Behandlung mit einer festsitzenden kieferorthopädischen 
Apparatur prävalent, vor allem, wenn gleichzeitig eine schlechte Mundhygiene besteht (Geiger et al. 1992, Hellwig et al. 2009). Die Prävention der Demineralisationen während einer Muttibracketbehandlung stellt trotz der Fortschritte in der Kariesprävention eine große Herausforderung dar (Bergstrand und Twetman 2011). Die Entstehung von WSL ist eine Folge anhaltender Plaque-Akkumulation neben den Brackets, die Hygienehindernisse darstellen (Gorelick et al. 1982, Artun und Brobakken 1986, O'Reilly und Featherstone 1987, Geiger et al. 1988, Øgaard et al. 1989). Festsitzende Apparaturen machen die konventionelle Mundhygiene nicht nur schwieriger, sie steigern auch die Anzahl der PlaqueRetentionsstellen an Glattflächen, die normalerweise als weniger kariesanfällig gelten (Tufekci et al. 2011). Das Problem, das sich daraus ergibt, ist, dass die Mundhygiene deshalb umso besser durchgeführt werden müsste, die KFO-Patienten mit Multibrackets sich aber meist im vorpubertären bis pubertären Alter befinden, in dem ihr Interesse an der Zahnpflege häufig sehr eingeschränkt ist (Sander et al. 2010). Für die Studie wurden Patienten gesucht, die kurz nach ihrer Entbänderung multibracket-induzierte WSL aufwiesen. Eben dieses Patientenklientel hat es zum Teil auch schwierig gestaltet, genug motivierte Patienten zu akquirieren, die die Bereitschaft zeigten, über 6 Monate regelmäßig zu den Untersuchungsterminen zu erscheinen.

\subsubsection{Der Infiltrant}

Der hier untersuchte Infiltrant ,Icon' ist ein lichthärtender Kunststoff, der für eine schnelle Penetration in die kapillaren Strukturen des Läsionskörpers optimiert wurde. Das Material ist sehr niedrigviskös, hat geringe Kontaktflächen mit dem Schmelz und eine hohe Oberflächenspannung (Meyer-Lueckel und Paris 2008). Diese Materialeigenschaften sind für eine komplette Penetration des Kunststoffinfiltranten in den Läsionskörper der Schmelzkaries wichtig. Des Weiteren demonstrierten Meyer-Lueckel und Paris die Inhibition der Kariesprogression von Läsionen nach der Applikation von Icon sowohl in vitro als auch in vivo (Meyer-Lueckel and Paris 2008, Mueller et al. 2006).

Die pseudointakte Schmelzoberfläche behindert das Penetrieren des Infiltranten in die Läsion. Deshalb muss diese Schicht durch Auftragen von 15\%-HCl-Gel zunächst entfernt werden. Im Gegensatz zur Schmelzmikroabrasion werden mit dieser Technik nur 30-40nm erodiert (Gugnani et al. 2012), was sie als mikroinvasiv gelten lässt. Betrachtet man die optischen Eigenschaften, so stellt man fest, dass Schmelz einen Brechungsindex von 1,62 hat. Die Mikroporositäten von Schmelzläsionen sind entweder mit einem Wassermedium, das den Brechungsindex 1,33 besitzt, oder mit Luft mit einem Brechungsindex 1 gefüllt. Der Unterschied der Brechungsindizes zwischen Schmelzkristallen und dem Medium innerhalb 
der Porositäten verursacht eine Lichtstreuung, die in einer weißlich-opaken Erscheinung der Läsion resultierten. Am deutlichsten erkennbar sind WSL in getrocknetem Zustand (Gugnani et al. 2012). Das Prinzip, die Schmelzläsionen durch Kunststoffinfiltration zu maskieren, basiert also auf den Veränderungen der Lichtstreuung innerhalb der Läsion (Paris et al. 2009).

Icon ist ein Kunststoff mit einem Brechungsindex von 1,51, der im Vergleich zum wässrigen Medium nicht verdunsten kann. Das macht den Unterschied des Brechungsindex zwischen Porositäten und Schmelz unbedeutend, und die Farbe der Läsion scheint der des angrenzenden Schmelzes ähnlich (siehe Abbildung 3, Seite 16).

Läsionen verlieren ihre weißlich-opake Farbe und vermischen sich gut mit der natürlichen Zahnstruktur (Kugel et al. 2009). Daher konnte eine sofortige Verbesserung des ästhetischen Erscheinungsbildes beobachtet werden.

\subsubsection{Anrauen des umliegenden Schmelzes}

Ohne die Vorbehandlung der häufig undurchlässigen Oberflächen-Schicht ist die Kunststoffinfiltration nur begrenzt möglich (Gray und Shellis 2002). Die relativ hohe Viskosität dentaler Kunststoffe (verglichen mit wässrigen Lösungen) und die kurze Behandlungszeit (verglichen mit der Fluoridbehandlung), verlangt relativ große Poren, um den Eintritt in den Läsionskörper gewährleisten zu können. Croll und Cavanaugh hatten die Idee, die Oberflächenschicht mechanisch mit einem Diamanten oder einem Polierstreifen zu entfernen (Croll und Cavanaugh 1986). Aber bei diesem Verfahren würden die Poren mit einer SmearLayer verschlossen werden und der Grad der Oberflächenreduktion wäre nur schwer zu kontrollieren. Mit der Schmelzätztechnik hingegen kann eine kontrollierte Reduktion dieser Oberflächenschicht erreicht werden. Meyer-Lueckel et al. haben die in der Zahnheilkunde gängige 37\%ige Phosphorsäure mit Hydrochloridsäuren verglichen (Meyer-Lueckel et al. 2007). Dabei stellte sich heraus, dass die 37\%ige Phosphorsäure, wenn sie für 30 bis 120 Sekunden aufgetragen wird, nicht dafür geeignet ist, die pseudointakte Oberfläche von natürlichen Läsionen zu entfernen. 15\%iges $\mathrm{HCl}$-Gel hingegen, das man für 90-120 Sekunden einwirken ließ, führte in den Versuchen zu einer praktisch komplett entfernten Oberflächenschicht. Aus diesem Grund hat man sich bei der Läsionsinfiltration für die Empfehlung 15\%iger $\mathrm{HCl}$-Säure entschieden. Ein Nebenbefund der vorliegenden Untersuchung war, dass bei einigen älteren, ausgeprägten WSL ein 120-Sekunden- oder auch 240-Sekunden-Ätzvorgang nicht zu einer vollständigen Entfernung der pseudointakten Schicht ausreicht, mit der Notwendigkeit weiterer Ätzvorgänge zur Entfernung der erkrankten Schicht. 
Zu bedenken ist weiterhin, dass man in der Behandlung mit Icon nicht verhindern kann, dass der umliegende Schmelz ähnlich behandelt wird wie die WSL. Beide Areale werden mit $\mathrm{HCl}$ Gel angeraut und mit dem Infiltranten behandelt. Die Anzahl der Ätzvorgänge in dieser Studie lag bei bis zu vier Wiederholungen mit je zwei Minuten. (Tabelle I, Seite 32). Das Risiko des übermäßigen Ätzens von in der Folge nicht geschützten Schmelzflächen beim kieferorthopädischen Bonding wurde bereits diskutiert und erforscht (Knösel et al. 2012b). Dies stellt jedoch bei der Infiltrationstechnik kein Problem dar, da die vorbehandelten Areale durch die Infiltration versiegelt und damit geschützt werden.

In der vorliegenden Studie wurde beobachtet, dass die einzige optische Veränderung in den infiltrierten AGS-Arealen die oben genannte Veränderung im CIE-b*-Wert war. Diese Veränderung konnte hier gleichermaßen in den WSL und in der Kontrollgruppe beobachtet werden. Vorherige In-vitro-Forschungen ergaben, dass es nach dem Ätzen im Vergleich zum umliegenden Schmelz keine signifikanten Unterschiede hinsichtlich der Erosionstiefen in der Läsion gibt (Meyer-Lueckel et al. 2007). Daraus kann geschlossen werden, dass das Ätzen und Infiltrieren des AGS die Struktur nicht sehr verändert und dass das Ätzen mehr vom porösen WSL-Schmelz als vom gesunden Schmelz entfernt. Das HCl-Ätzgel wurde daher auch in einer hohen Viskosität hergestellt, so dass man es relativ sicher nur auf die zu ätzenden Bereiche auftragen kann, wodurch das Ätzen des umliegenden Schmelzes minimalisiert wird. Des Weiteren kann durch die Viskosität die Penetration in den Läsionskörper hinein verhindert werden, welche zu schweren, irreversiblen Strukturveränderungen führen könnte (Meyer-Lueckel et al. 2007). Insgesamt wird die Methode der Kunststoffinfiltration als mikroinvasiv (also substanzerhaltend) und vor allem weniger invasiv als herkömmliches restauratives Versorgen der WSL angesehen. Durch die Infiltration sind ästhetisch ansprechendere Resultate möglich als sie mit der Abrasion durch die Zahnbürste und Remineralisation alleine erzielt werden können (Willmot 2004, Wiegand et al. 2007). Auch verglichen mit der rein ästhetischen Behandlung der WSL durch Bleaching, ist die Methode der Infiltration wirkungsvoller, nachhaltiger und umfassender (Knösel et al. 2007).

\subsubsection{Der Faktor der Läsionstiefe, -ausdehnung und -alter der WSL}

Während der Studie war zu beobachten, dass bei manchen älteren und tieferen Läsionen eine mehrfache Wiederholung des Ätzvorgangs notwendig war, um zu akzeptablen Infiltrierungen mit ästhetischen Ergebnissen zu kommen. Allerdings kam es bei diesen tiefen WSL vor, dass sie trotz längerer Ätzdauer zwar infiltriert und optisch verbessert wurden, aber trotzdem deutlich sichtbar blieben (Abbildung 16, Seite 58). 
Das lässt sich dadurch erklären, dass sich eine Läsion zeitabhängig vertieft, während die Oberflächenschicht mit der Zeit remineralisiert, was zum einen das Durchdringen der Oberfläche und zum anderen das Penetrieren bis in die Tiefe der Läsion erschwert.

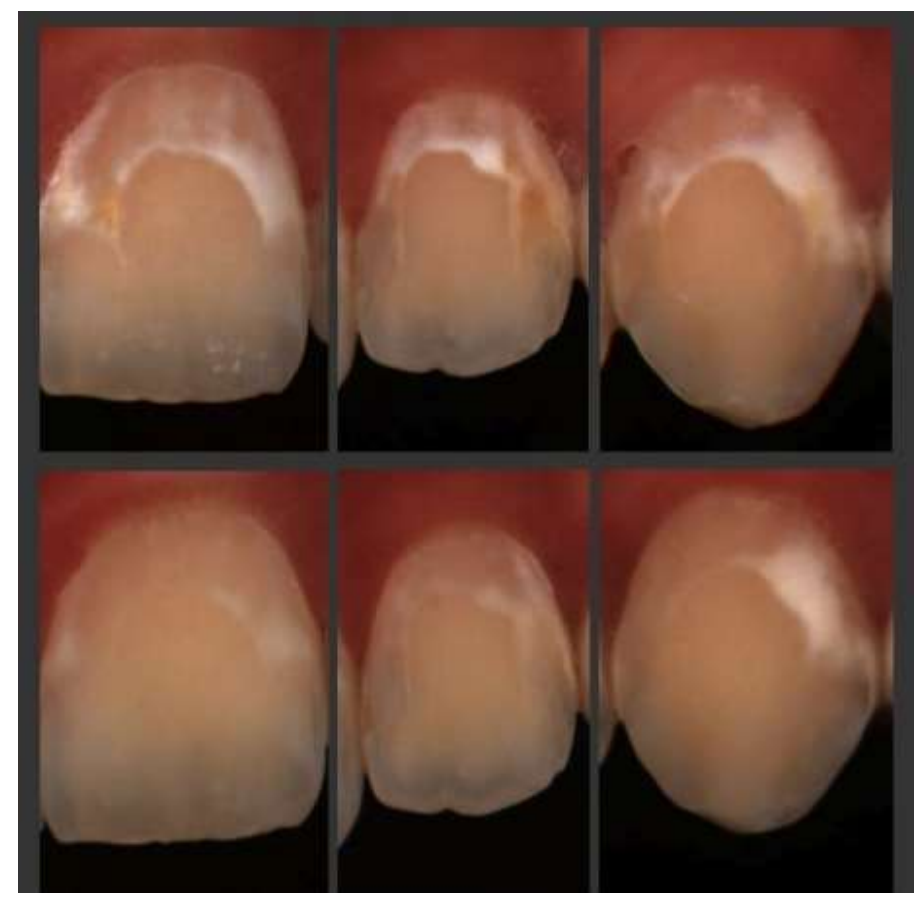

Abbildung 16: Während in den meisten Fällen die Läsionen nach der Infiltration kaum noch sichtbar sind, was durch den ähnlichen Brechungsindex von Schmelz $(R I=1,62)$ und dem Infiltranten $(\mathrm{Rl}=1,51)$ zu erklären ist, bleiben einige Opazitäten in manchen Fällen auffällig.

Obere Reihe: t0, Baseline

Untere Reihe: $\mathrm{t} 1$, nach Infiltration

(Knösel et. al 2013 S.95)

Kleinere und oberflächlicher lokalisierte Läsionen wurden nach ästhetischen Aspekten effektiver behandelt. Die Dauer der Existenz der WSL spielt eine maßgebliche Rolle: Die Zeitspanne zwischen Entbänderung und Infiltration sollte so kurz wie möglich sein, um Remineralisierung und vor allem die Oberflächenveränderungen durch tägliches Zähneputzen zu verhindern. Je weniger die porösen Schmelzoberflächen vor der Infiltration wieder remineralisieren, umso besser wird sich das ästhetisches Ergebnis darstellen. Ähnliche Beobachtungen konnten in vereinzelten Fallberichten beschrieben werden (Neuhaus et al. 2010). 


\subsection{Ergebnisse}

\subsubsection{Kontrollierte Farb- und Helligkeitsentwicklung über 6 Monate (von Baseline bis t5)}

Das Hauptergebnis der vorliegenden Untersuchung ist, dass ein optischer Angleich der Farbe der infiltrierten WSL an die der umgebenden Schmelzareale erfolgte, der über sechs Monate ohne signifikante Veränderungen in vivo farbstabil blieb. In der Gruppe der unbehandelten Zähne gab es in dem halben Jahr keine signifikanten Veränderungen im Vergleich zu Baseline. Dies ist die erste strukturierte, kontrollierte randomisierte Studie, die sich dieser Fragestellung widmet. Im an die WSL angrenzenden Schmelz war keine signifikante Veränderung der Helligkeit $\left(\mathrm{CIE}-\mathrm{L}^{*}\right)$ und der Rot-Grün-Farbachse (CIE-a*) festzustellen, während signifikante Farb- und Helligkeitsveränderungen in den infiltrierten WSL festgestellt wurden. In den WSL sowie im Schmelz der infiltrierten Zähne und der Kontrollgruppe konnten zwar signifikante Veränderungen in der Blau-Gelb-Achse ( $b^{*}$-Wert) festgestellt werden, was jedoch ähnlich bereits in der Literatur über sechs Monate in den b*Werten der Zähne unbehandelter Patienten berichtet wurde (Sluzker et al. 2011). Daher kann eine Schwankung dieses Wertes über sechs Monate als normal betrachtet werden. Es ist auch fraglich, ob die mittlere Abweichung von -0.74 Einheiten auf der b*-Skala klinisch relevant ist. Obwohl sie statistisch als signifikant gilt, ist der $\Delta \mathrm{E}$ Schwellenwert für die klinische Sichtbarkeit mit 3.7 CIE-L*a*b* $\Delta$ E-Einheiten angegeben (Johnston und Kao 1989). Ergänzend ist hinzuzufügen, dass alle in dieser Studie behandelten Patienten mit dem Behandlungsergebnis zufrieden waren.

\subsubsection{Baselinekontrollierte Farb- und Helligkeitsentwicklung über 12 Monate}

Es konnte nach einem Jahr keine signifikante Farbveränderung zwischen t5 und t6 festgestellt werden, obwohl sich die $\Delta \mathrm{E}$ - Differenz (WSL/ASE) bei t6 im Vergleich zu t5 etwas vergrößert hat. Die Farbveränderung von t0 zu t6 blieb weiterhin signifikant. Da der Wert der $\Delta \mathrm{E}$ - Differenz, wie oben beschrieben, weiterhin unter 3,7 Einheiten liegt (Johnston und Kao 1989), lässt sich daraus schließen, dass auch in den zweiten sechs Monaten keine für das menschliche Auge wahrnehmbare Farbveränderung stattgefunden hat und das Infiltrationsergebnis über ein Jahr stabil geblieben ist. 


\subsubsection{Zusammengefasste Farb- und Helligkeitsentwicklung}

Es gab durch die Infiltration eine farbstabile Angleichung der WSL-Farbe an die des umliegenden Schmelzes, ohne dass sich diese signifikant über sechs Monate veränderte.

Die Nullhypothese, dass es keine signifikante Farb- und Helligkeitsveränderung (CIE-L*a*b* $\Delta \mathrm{E}$-Werte) zwischen den WSL und den daran angrenzenden Schmelzarealen nach der Infiltration im Vergleich zur nicht behandelten Kontrollgruppe gibt, wurde zurückgewiesen.

Die $\Delta \mathrm{E}$-Werte haben sich in der behandelten Gruppe signifikant reduziert (Tabelle IV, Seite 39; Icon (Mittelwert) von $8.14 \pm 4.59$ zu $5.59 \pm 2.82$; Kontrollgruppe (Mittelwert) von $7.9 \pm 4.51$ zu $7.6 \pm 4.51$ ). Diese Differenz in der Icon-Gruppe ist klinisch relevant, da es sie den Schwellenwert erreicht, der die Kapazität des menschlichen Auges kennzeichnet (Johnston und Kao 1989), wenngleich in manchen Fällen die Opazität noch sichtbar war (Abbildung 16, Seite 58). Die einzige nennenswerte Veränderung in der Kontrollgruppe war ein vorübergehender Anstieg in der Helligkeit bei der t1-Messung, die auf die Infiltrationsbehandlung folgt. Diese Veränderung ergibt sich durch die Austrocknung der kompletten Zahnreihen, also auch der Kontrollzähne, während der Infiltration. Durch das Trockenhalten der Zähne mit Sauger, Watte und Wangen-Lippenschild werden die Zähne temporär für ca. 24 Stunden aufgehellt. Der Effekt wurde in der Literatur beschrieben (Russel et al. 2000). In den folgenden Untersuchungsterminen war die gestiegene Helligkeit entsprechend nicht mehr zu verzeichnen.

\subsection{Perspektiven für weitergehende Forschung}

Da dies die erste kontrollierte In-vivo-Langzeitstudie zum Thema der Langzeitstabilität der optischen Verbesserungen von WSL durch Infiltration darstellt, werden sich Folgeuntersuchungen der hier infiltrierten Zähne anschließen. Dies beinhaltet neben einer Untersuchung der Farbstabilität auch eine Überwachung der Integrität der mit-infiltrierten angrenzenden Schmelzareale. Gut strukturierte In-vivo-Projekte zur Prophylaxe von WSL während der kieferorthopädischen Behandlung sowie zur Infiltration aufgetretener WSL bereits während der Multibracketbehandlung werden als sinnvoll erachtet. 


\subsection{Schlussfolgerung}

Auf den Ergebnissen dieser Studie basierend können folgende Schlussfolgerungen gezogen werden:

Das Erscheinungsbild demineralisierter Zähne verbessert sich durch die Infiltration der WSL. Dieses Ergebnis zeigt über den Zeitraum eines Jahres eine adäquate Stabilität. Weder die Farbe noch die Helligkeit des angrenzenden gesunden Schmelzes wird signifikant verändert. Lediglich der $b^{*}$-Wert der Behandlungs- und Kontrollgruppe zeigte eine signifikante Veränderung, jedoch in einer Größenordnung, die vom menschlichen Auge nicht wahrnehmbar ist, und daher als klinisch irrelevant eingestuft wird.

Für die Infiltrationsbehandlung der WSL spielt deren Alter, Oberflächenstruktur und Tiefe eine entscheidende Rolle: Je kleiner die Läsion ist und je früher nach Entfernung der Brackets infiltriert wird, umso weniger Konditionierungsintervalle werden benötigt und umso weniger WSL-Opazitäten bleiben sichtbar. Eine Infiltrationsbehandlung sollte daher möglichst zeitnah nach Entfernung der Multibracket-Apparatur durchgeführt werden. 


\section{Zusammenfassung}

White-Spot-Läsionen (WSL), die sich häufig als unerwünschte Nebenwirkung einer festsitzenden kieferorthopädischen Behandlung auf den Glattflächen des Zahnschmelzes ausbilden, stellen ein frühes Kariesstadium und eine ästhetische Beeinträchtigung im Frontzahnbereich dar. Durch die neuartige Methode der Läsions-Infiltration (Icon, DMG Chemisch-Pharmazeutische Fabrik GmbH, 22547 Hamburg, Deutschland) kann ein Fortschreiten der Läsion verhindert werden; gleichzeitig ergibt sich durch den ähnlichen Lichtbrechungsindex von gesundem Schmelz und Infiltrant ein optisches Angleichen der WSL an den angrenzenden gesunden Schmelz (AGS). Bislang existierten zu diesem Thema der WSL-Camouflage lediglich In-vitro-Untersuchungen an infiltrierten Rinderschmelzproben bzw. Kurzzeit-Fallberichte. Primäres Ziel der vorliegenden Arbeit ist daher die erstmalige Bestimmung der Beständigkeit der ästhetischen Angleichung von White-Spot-Läsionen (WSL) durch Infiltration (Icon) im Vergleich zu unbehandelten WSL über 6 Monate in vivo. Das sekundäre Ziel ist die Nachuntersuchung der infiltrierten Läsionen nach 12 Monaten.

Methode: Multibracket-induzierte WSL von 20 konsekutiven Probanden der Abt. Kieferorthopädie der Universitätsmedizin Göttingen ( $n_{\text {WSL total }}=219 ; n_{\text {Icon }}=111 ; n_{\text {Kontrolle }}=108$ ) wurden nach vorangegangener Schmelzkonditionierung $(15 \% \mathrm{HCl}-\mathrm{Gel})$ nach Herstellerangaben infiltriert. Farbe und Helligkeit (CIE-L*a*b*) der WSL wurden im Vergleich zu umgebenden, gesunden Schmelzarealen spektrophotometrisch (ShadePilot, Degudent, Hanau-Wolfgang, Deutschland) in einem randomisierten, split-mouth-kontrollierten Design erfasst zu den Zeitpunkten: Vor Infiltration (Baseline, t0), nach 1 Tag (t1), 1 Woche (t2), 4 Wochen (t3), 3 Monaten (t4), 6 Monaten (t5). Die Kontrollquadranten wurden als Teil der Vereinbarung mit den Probanden nach sechs Monaten ebenfalls infiltriert. Die infiltrierten WSL von 9 Probanden ( $n_{\text {Icon }}=49$; response rate: 45\%) konnten nach 12 Monaten nachuntersucht werden (t6). Der Einfluß von Infiltration und Zeit auf die Farb- und Helligkeitsveränderungen ( $\Delta \mathrm{E}$-Werte) wurden mit Hilfe multi-faktorieller ANOVA und Posthoc-Tests mit einem a-Level von 5\% und einer Power von $80 \%$ (t5) bestimmt. Ergebnisse: Sowohl Infiltration als auch die Zeitdauer hatten global einen hoch signifikanten Einfluss auf die $\Delta \mathrm{E}$-Werte. WSL glichen sich nach Infiltration an die umgebenden gesunden Schmelzareale an; dieser Effekt war über 12 Monate farbstabil ohne signifikante Veränderung. An den unbehandelten Kontrollzähnen ergaben sich im Vergleich zu den Baseline-Werten keine signifikanten Farb- oder Helligkeitsveränderungen. Schlussfolgernd kann die WSL-Infiltration als ein praxistaugliches Konzept zur langfristigen Verbesserung der Ästhetik demineralisierter Frontzahnflächen empfohlen werden. 


\section{Literaturverzeichnis}

Aas JA, Paster BJ, Stokes LN, Olsen I, Dewhirst FE (2005): Defining the normal bacterial flora of the oral cavity. J Clin Microbiol $\underline{43}, 5721-5732$

Al-Khateeb S, Forsberg CM, de Josselin de Jong E, Angmar-Mansson B (1998): A longitudinal laser fluorescence study of white spot lesions in orthodontic patients. Am J Orthod Dentofacial Orthop $\underline{113}$, 595-602

Andrews LF (1979): The straight-wire appliance. Br J Orthod.

Archegas LR, Freire A, Vieira S, Caldas DB, Souza EM (2011): Colour stability and opacity of resin cements and flowable composites for ceramic veneer luting after accelerated ageing. J Dent $\underline{39}(\underline{11}), 804-810$

Artun J, Thylstrup A (1986): Clinical and scanning electron microscopic study of surface changes of incipient caries lesions after debonding. Scand J Dent Res 94,193-201

Artun J, Brobakken BO (1986): Prevalence of carious white spots after orthodontic treatment with multibanded appliances. Eur J Orthod $\underline{8}, 229-34$

Backer Dirks O (1966): The clinical testing of agents for the prevention of dental caries. Adv Fluorine Res. $\underline{4}, 1-2$

Balenseifen JW, Madonia JV (1970): study of dental plaque in orthodontic patients. J Dent $\operatorname{Res} \underline{49}$,

320-324

Benson PE, Pender N, Higham SM (1999): An in situ caries model to study demineralization during fixed orthodontics. Clin Orthod Res $\underline{2}, 143-153$.

Benson PE, Parkin N, Millett DT, Dyer FE, Vine S, Shah A (2004): Fluorides for the prevention of white spots on teeth during fixed brace treatment. Cochrane Database Syst Rev 3: CD003809 
Bergstrand F, Twetman S (2011): A review on prevention and treatment of post-orthodontic white spot lesions - evidence based methods and emerging technologies. Open Dent $\mathrm{J} \underline{5}$, 158-162

Berns RS, Billmeyer FW, Saltzman M.: Billmeyer and Saltzman's principles of color technology. 3rd ed, Wiley, New York 2000

Bishara SE, Ostby AW (2008): White Spot Lesions: Formation, Prevention, and Treatment. Semin Orthod 14(3), 174-182

Bolt RA, ten Bosch JJ, Coops JC (1994): Influence of window size in small-window color measurement, particularly of teeth. Physics in Medicine and Biology $\underline{39}, 1133-1142$

Bruker MO, Ziegelbecker R: Vorsicht Fluor!: Das Kariesproblem. Fluoridtabletten, Fluoridlacke, Kochsalzfluoridierung, Trinkwasserfluoridierung. 9. Auflage; emu-Verlags- und Vertriebs-GmbH, Lahnstein 2000

Buren JL, Staley RN, Wefel J, Quian F (2008): Inhibition of enamel demineralisation by an enamel sealant, Pro Seal: an in-vitro study. Am J Orthod Dentofacial Orthop $\underline{133}, 88-94$

Burne RA, Marquis RE (2000): Alkali production by oral bacteria and protection against dental caries. FEMS Microbiol Lett 193, 1-6

Buschang PH, Campbell PM, Julien KC (2013): Prevalence of white spot lesion formation during orthodontic treatment. Angle Orthod 83(4), 641-647

Busscher HJ, van der Mei HC (1997): Physico-chemical interactions in initial microbial adhesion and relevance for biofilm formation. Adv Dent Res 11, 24-32

Chang HS, Walsh LJ, Freer TJ (1997): Enamel demineralization during orthodontic treatment. Aetiology and prevention. Aust Dent J $\underline{42}, 322-327$

Commission Internationale de l'Eclairage (CIE) (2004): Colorimetry, 3rd ed. Publication CIE No. 15.3. Vienna, Austria: Central Bureau of the CIE 
Croll TP, Cavanaugh RR (1986): Enamel color modification by controlled hydrochloric acidpumice abrasion. I. Technique and examples. Quintessence Int $\underline{17}$, 81-87

Davila JM, Buonocore MG, Greeley CB, Provenza DV (1975): Adhesive penetration in human artificial and natural white spots. J Dent Res $\underline{54}, 999-1008$

Demito CF, Vivaldi-Rodrigues G, Ramos AL, Bowman SJ (2004): The efficacy of a fluoride varnish in reducing enamel demineralization adjacent to orthodontic brackets: an in vitro study. Orthod Craniofac Res $\underline{7}, 205-210$

DGZMK (2000): Empfehlungen zur Kariesprophylaxe mit Fluoriden. Wissenschaftliche Stellungnahme (Gülzow HJ, Hellwig E, Hetzer G), URL:http://www.dgzmk.de

Donly KJ, Istre S, Istre T (1995): In vitro enamel remineralization at orthodontic band margins cemented with glass ionomer cement. Am J Orthod Dentofacial Orthop 107, 461464

Dubroc GC Jr, Mayo JA, Rankine CA (1994): Reduction of caries and of demineralization around orthodontic brackets: effect of a fluoride-releasing resin in the rat model. Am J Orthod Dentofacial Orthop 106, 583-537

Emilson CG, Krasse B (1985): Support for and implications of the specific plaque hypothesis. Scand J Dent Res $\underline{93}$, 96-104

Fani G, Vichi A, Davidson CL (2007): Spectrophotometric and visual shade measurements of human teeth using three shade guides. Am J Dent 20, 142-146

Featherstone JD (2000): The science and practice of caries prevention. J Am Dent Assoc $\underline{131}, 887-899$

Fejerskov O, Nyvad B, Kidd EAM (2003) Clinical and histological manifestations of dental caries. In: Dental Caries-The Disease and Its Clinical Management. Ames, lowa: Blackwell Munksgaard $\underline{5}, 72-97$

Fischer C, Lussi A, Hotz P (1995): Kariostatische Wirkungsmechanismen der Fluoride. Schweiz Monatsschr Zahnmed 105, 311-317 
Fitzgerald RJ, Keyes PH (1960): Demonstration of the etiologic role of streptococci in experimental caries in the hamster. J Am Dent Assoc 61, 9-19

Franke G, Baume LJ (1976): Klassifizierung epidemiologischer Studien über Zahnkaries und Definition verwandter Begriffe, Herausgegeben von Mitgliedern der Arbeitsgruppe 1 der Kommission zur Klassifizierung und Normung von Statistiken über Gebissbefunde der Fédération Dentaire Internationale (FDI). Int Dent J 26, 73-95

Gängler P, Hoffmann, T, Willershausen, B., Schwenzer, N, Ehrenfeld, M: Zahn-Mund-KieferHeilkunde. Lehrbuchreihe zur Aus- und Weiterbildung: Konservierende Zahnheilkunde und Parodontologie, Thieme, Stuttgart 2010

Geiger AM, Gorelick L, Gwinnett AJ, Griswold PG (1988): The effect of a fluoride program on white spot formation during orthodontic treatment. Am J Orthod Dentofacial Orthop 93(1), 2937

Geiger AM, Gorelick L, Gwinnett AJ, Benson BJ (1992): Reducing white spot lesions in orthodontic populations with fluoride rinsing. Am J Orthod Dentofacial Orthop 101(5), 403407

Glazer HS (2009): Treating white spots: new caries infiltration technique. Dent Today $\underline{28}(10)$, 82, 84-85

Gorelick L, Geiger AM, Gwinnett AJ (1982): Incidence of white spot formation after bonding and banding. Am J Orthod 81, 93-98

Gorton J, Featherstone JD (2003): In vivo inhibition of demineralization around orthodontic brackets. Am J Orthod Dentofacial Orthop 123, 10-14

Gray GB, Shellis P (2002): Infiltration of resin into white spot caries-like lesions of enamel: an in vitro study. Eur J Prosthodont Resort Dent 10, 27-32

Griffin SO, Oong E, Kohn W, Vidakovic B, Gooch BF, Bader J, Clarkson J, Fontana MR, Meyer DM, Rozier RG, Weintraub JA, Zero DT (2008): The effectiveness of sealants in managing caries lesions. J Dent Res $\underline{87}, 169-174$ 
Gugnani N, Pandit IK, Gupta M, Josan R (2012): Caries infiltration of noncavitated white spot lesions: A novel approach for immediate esthetic improvement. Contemp Clin Dent $\underline{3}: 199$ 202

Hadler-Olsen S, Sandvik K, El-Agroudi MA, Ogaard B (2011): The incidence of caries and white spot lesions in orthodontically treated adolescents with a comprehensive caries prophylactic regimen-a prospective study. Eur J Orthod [Epub ahead of print]

Hammad IA (2003): Intrarater repeatability of shade selections with two shade guides. J Prosthet Dent $\underline{89}, 50-53$

Hecht E: Optics. 4th ed; Addison-Wesley Longman, Boston 2002

Hellwig E (1996): Fluoride - Chemie und Biochemie. Dtsch Zahnarztl Z $\underline{51}$, 638-648

Hellwig E., Klimek J., Attin T: Einführung in die Zahnerhaltung. 5. Auflage; Deutscher Zahnärzte-Verlag, Köln 2009: 3-8, 15-33, 98-102, 111-142

Holmen L, Thylstrup A, Artun J (1987): Surface changes during the arrest of active enamel carious lesions in vivo. A scanning electron microscope study. Acta Odontol Scand $\underline{45}, 383-$ 390

Hotz PR (1996): Anwendung der Fluoride in der Zahnmedizin. Dtsch Zahnärztl Z 1ㅗ, 649-653

Inokoshi S, Burrow MF, Kataumi M (1996): Opacity and color changes of tooth-colored restorative materials. Oper Dent $\underline{21}, 73-80$

Ishiwata T (1986): A study of color in prosthodonticsexamination using spectroradiometry. Jpn Prosthodont Soc (Nippon Hotetsu Shikka Gakkai Zasshi) 으, 652-664

Ishikawa-Nagai S, Da Silva JD, Weber HP, Park SE (2007): Optical phenomenon of periimplant soft tissue. Part II. Preferred implant neck color to improve soft tissue esthetics. Clinical oral implants research $\underline{18}, 575-80$

Johnston WM (2009): Color measurement in dentistry. J Dent $\underline{37}$ (Supplement 1), e2-6 
Johnston WM, Kao EC (1989): Assessments of appearance match by visual observation and clinical colorimetry. J Dent Res $\underline{68(5)}, 819-22$

Joiner A, Jones NM, Raven SJ (1995): Investigation of factors influencing stain formation utilizing an in situ model. Advances in Dental Research $\underline{9}, 471-476$

Joiner A (2004): Tooth colour: a review of the literature. J Dent 32, 3-12

Kalha A (2004): Some evidence that fluoride during orthodontic treatment reduces occurrence and severity of white spot lesions. Evid Based Dent $\underline{5}, 98-99$

Karamouzos A, Papadopoulos MA, Kolokithas G, Athanasiou AE (2007): Precision of in vivo spectrophotometric color evaluation of natural teeth. Journal of Oral Rehabilitation $\underline{34,613}$ 621.

Keyes PH, Fitzgerald RJ (1962): Dental caries in the Syrian hamster. IX. Arch Oral Biol $\underline{7}$, 267-277

Kidd EAM, van Amerongen JP, van Amerongen WE (2008): The role of operative treatment in caries control; in Fejerskov O, Kidd EAM (eds): Dental Caries: The Disease and Its Clinical Management. Oxford, Blackwell Munksgaard, 2, 355-365

Kim HS, Um CM (1996): Color differences between resin composites and shade guides. Quintessence Int 27, 559-67.

Kleber CJ, Milleman JL, Davidson KR, Putt MS, Triol CW, Winston AE (1999): Treatment of orthodontic white spot lesions with a remineralizing dentifrice applied by toothbrushing or mouth trays. J Clin Dent $\underline{10}(1), 44-49$

Klimm W: Kariologie, Carl Hauser Verlag, München, Wien 1997: 48, 126-131, 206-207

Kneist S, Zingler S, Lux C (2008): Thearapiebegleitende Maßnahmen zur Kontrolle des Karies-und Demineralisationsrisikos bei kieferorthpädischen Patienten. ZWR Dtsch Zahnärztebl 117, 218-226 
Knösel M, Attin R, Becker K, Attin T (2007): External Bleaching Effect on the Color and Luminosity of Inactive White-Spot Lesions after Fixed Orthodontic Appliances. Angle Orthod $\underline{77}, 646-652$

Knösel M, Attin R, Jung K, Brunner E, Kubein-Meesenburg D, Attin T (2009): Digital Image Color Analysis compared to Direct Dental CIE $\left(\mathrm{L}^{*}, \mathrm{a}^{*}, \mathrm{~b}^{*}\right)$ Colorimeter Assessment under Different Ambient Conditions. Am J Dent 22(2), 67-72

Knösel M, Reus M, Rosenberger A, Attin T, Ziebolz D (2011): Durability of bleaching results achieved with $15 \%$ carbamide peroxide and $38 \%$ hydrogen peroxide in vitro. Eur J Esthet Dent 6(3), 342-56.

Knösel M, Reus M, Rosenberger A, Ziebolz D (2012a): A novel method for testing the veridicality of dental colour. Eur J Orthod 34(1), 19-24

Knösel M, Bojes M, Jung K, Ziebolz D (2012b): Increased susceptibility for white spot lesions by surplus orthodontic etching exceeding bracket base area. Am J Orthod Dentofacial Orthop $\underline{141(5)}, 574-582$

Knösel M, Eckstein A, Helms H-J (2013): Durability of esthetic improvement following Icon resin infiltration of multibracket-induced white spot lesions compared with no therapy over 6 months: A single-center, split-mouth, randomized clinical trial. Am J Orthod Dentofacial Orthop $\underline{144}$, 86-96

Krasse B (1966): Human streptococci and experimental caries hamsters. Arch Oral Biol 11, 429-436

Kuehni RG (2002): The early development of the Munsell system. Color Research and Application 27, 20-27

Kuehni RG, Marcus RT (1979): A experiment in visual scaling of small color difference. Color Research and Application 4, 83-91

Kugel G, Arsenaul P, Papas A (2009): Treatment modalities for caries management, including a new resin infiltration system. Compend Contin Educ Dent 30, 1-10 
Lundstrom F, Krasse B (1987): Streptococcus mutans and lactobacilli frequency in orthodontic patients; the effect of chlorhexidine treatments. Eur J Orthod $\underline{9}, 109-116$

Marsh PD (1995): Effect of fluorides on bacterial metabolism. In: Bowen WH: Relative efficacy of sodium monofluorphosphate as anti-caries agent in dentifrices. Royal Soc Med Press, London: 9

Marsh P, Martin MV: Orale Mikrobiologie, Thieme, Stuttgart 2003

Marsh PD, Moter A, Devine DA (2011): Dental plaque biofilms: communities, conflict and control. Periodontol $2000 \underline{55}, 16-35$

Mattousch TJ, van der Veen MH, Zentner A (2007): Caries lesions after orthodontic treatment followed by quantitative light-induced fluorescence: a 2-year follow-up: Eur $\mathrm{J}$ Orthod $\underline{29}, 294-298$

McLaren K (1987): Colour space, colour scales and colour difference. In: McDonald R, editor. Colour physics for industry. Huddersfield: H. Charlesworth \& Co Ltd; 1987, 97-115

Melrose CA, Appleton J, Lovius BB (1996): A scanning electron microscopic study of early enamel caries formed in vivo beneath orthodontic bands. Br J Orthod $\underline{23}, 43-47$

Meyer-Lueckel H, Paris S (2008a): Improved resin infiltration of natural caries lesions. J Dent $\underline{87}, 1112-1116$

Meyer-Lueckel H, Paris S (2008b): Progression of artificial enamel caries lesions after infiltration with experimental light curing resins. Caries Res $\underline{42}, 117-124$

Meyer-Lueckel H, Paris S, Kielbassa AM (2007): Surface layer erosion of natural caries lesions with phosphoric and hydrochloric acid gels in preparation for resin infiltration. Caries Res $\underline{41(3)}$, 223-30

Mueller J, Meyer-Lueckel H, Paris S, Hopfenmueller W, Kielbassa AM (2006): Inhibition of lesion progression by penetration of resins in vitro: Influence of the application procedure. Oper Dent $\underline{31}, 338-345$ 
Neuhaus KW, Graf M, Lussi A, Katsaros C (2010): Late infiltration of post-orthodontic white spot lesions. J Orofac Orthop $\underline{71(6)}, 442-447$

Newbrun E (1989): Cariology. 3rd edn. Chicago. Quintessence1989, 29-61

Nuttal NM, Pitts NB (1990): Restorative treatment thresholds reported to be used by dentists in Scotland. Br Dent J 169, 119-126

O'Brien WJ, Johnston WM, Fanian F (1990): Double-layer color effects in porcelain systems. J Dent Res 64, 940-943

O'Brien WJ, Groh CL, Boenke KM (1990b): A new, small-colordifference equation for dental shades. J Dent Res $\underline{69}, 1762-1764$

Øgaard B (1988): Prevalence of white spot lesions in 19-year-olds: a study on untreated and orthodontically treated persons 5 years after treatment. Am J Orthod Dentofacial Orthop $\underline{96}$, 423-427

Øgaard B, Ten Bosch JJ (1994): Regression of white spot enamel lesions. A new optical method for quantitative longitudinal evaluation in vivo. Am J Orthod Dentofacial Orthop 106, 238-242

Øgaard B, Rolla G, Arends J (1988): Orthodontic appliances and enamel demineralization. Part 1. Lesion development. Am J Orthod Dentofacial Orthop 94:68-73

Øgaard B, Larsson E, Henriksson T, Birkhed D, Bishara SE (2001): Effects of combined application of antimicrobial and fluoride varnishes in orthodontic patients. Am $\mathrm{J}$ Orthod Dentofacial Orthop $\underline{120}, 28-35$

Okubo SR, Kanawati A, Richards MW (1998): Evaluation of visual and instrument shade matching. J Prosthet Dent $\underline{80}$, 642-648

O'Reilly MM, Featherstone JDB (1987): Decalcification and remineralization around orthodontic appliances: an in vivo study. Am J Orthod Dentofacial Orthop 92(1), 33-40 
Orland FJ, Blayney JR, Harrison RW, Reyniers JA, Trexler PC, Wagner M, Gordon HA, Luckey TD (1954): Use of the germfree animal technic in the study of experimental dental caries. I. Basic observations on rats reared free of all microorganisms. J Dent Res $\underline{33}, 147-$ 174

Orland FJ, Blayney JR, Harrison RW, Reyniers JA, Trexler PC, Ervin RF, Gordon HA, Wagner M (1955): Experimental caries in germfree rats inoculated with enterococci. J Am Dent Assoc 무, 259-272

Palamara J, Phakey PP, Rachinger WA, Orams HJ (1986): Ultrastructure of the intact surface zone of white spot and brown spot carious lesions in human enamel. J Oral Pathol $\underline{15}, 28-35$

Paris S, Meyer-Lueckel H (2009): Masking of labial enamel white spot lesions by resin infiltration-a clinical report. Quintessence Int $\underline{40(9)}$, 713-728

Paris S, Meyer-Lueckel H (2010): Inhibition of Caries Progression by Resin Infiltration in situ. Caries Res $\underline{44}, 47-54$

Paris S, Meyer-Lueckel H, Colfen H, Kielbassa AM (2007a): Resin infiltration of artificial enamel caries lesion with experimental light-curing resins. Dent Mater J $\underline{26}, 582-588$

Paris S, Meyer-Lueckel H, Kielbassa AM (2007b): Resin infiltration of natural caries lesions. J Dent Res 6ㅜ, 662-666

Paris S, Hopfenmuller W, Meyer-Lueckel H (2010): Resin infiltration of caries lesions: an efficacy randomized trial. J Dent Res $\underline{89(8)}$, 823-826

Paul S, Peter A, Pietrobon N (2002): Visual and Spectrophotometic Shade Analysis of Human Teeth. J Dent Res $\underline{81}, 578-582$

Pitts NB (2004): Are we ready to move from operative to non-operative/ preventive treatment of dental caries in clinical practice? Caries Res $\underline{38}, 294-304$

Plagmann H-C: Lehrbuch der Parodontologie. Hanser, München-Wien 1998, 43-46 
Qvist V (2008), Longevity of restaurations: ,the death spriral'. In: Dental caries: the disease and its clinical management. Fejerskov O, Kidd EAM, editors. Oxford, UK: Black Munksgaard 2008, 444-455

Richter AE, Arruda AO, Peters MC, Sohn W (2011): Incidence of caries lesions among patients treated with comprehensive orthodontics. Am J Orthod Dentofacial Orthop $\underline{139}$, 65764

Rocha Gomes Torres C, Borges AB, Torres LM, Gomes IS, de Oliveira RS (2011): Effect of caries infiltration technique and fluoride therapy on the colour masking of white spot lesions.

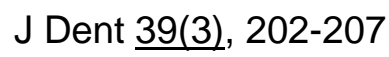

Rodda JC (1983): Impregnation of caries-like lesions with dental resins. N Z Dent J $\underline{79}, 114$ 117

Russell MD, Gulfraz M, Moss BW (2000): In vivo measurement of colour changes in natural teeth. Journal of Oral Rehabilitation 27, 786-792

Ruyter IE, Nilner K, Moller B (1987): Color stability of dental composite resin materials for crown and bridge veneers. Dent Mater $\underline{3}, 246-251$.

Sander F G, Schwenzer N, Ehrenfeld M: Zahn-Mund-Kieferheilkunde Kieferorthopädie. 2. Auflage; Georg Thieme Verlag, Stuttgart, New York 2010, 44-45

Seghi RR, Johnston WM, O'Brien WJ (1989): Performance assessment of colorimetric devices on dental porcelains. J Dent Res $\underline{68}, 1755-1759$

Shannon IL (1981): Prevention of decalcification in orthodontic patients. J Clin Orthod 15, 694-706

Sluzker A, Knösel M, Athanasiou AE (2011): Sensitivity of digital dental photo CIE L*a*b* analysis compared to spectrophotometer clinical assessments over 6 months. Am J Dent $\underline{24(5)}, 300-4$

Smith T, Guild J (1931): The C.I.E. colorimetric standards and their use. Trans Opt Soc $\underline{33}$, 73-134 
Stahl J, Zandona AF (2007): Rationale and protocol for the treatment of non-cavitated smooth surface carious lesions. Gen Dent $\underline{55(2), 105-111}$

Stephan RM (1940): Changes in hydrogen-ion concentration on tooth surfaces and in caries lesions. J Am Dent Assoc 27, 718-723

Subramaniam K., Siswomihardjo W, Sunarintyas S (2005): The effect of different concentrations of Neem (Azadiractha indica) leaves extract on the inhibition of Streptococcus mutans (In vitro). Maj Ked Kigi Dent J $\underline{38}, 1769$.

Ten Bosch JJ, Coops JC (1995): Tooth color and reflectance as related to light scattering and enamel hardness. J Dent Res $\underline{74}$, 374-380

Ten Cate JM, Duijsters PP (1983): Influence of fluoride in solution on tooth demineralization. II. Microradiographic data. Caries Res $\underline{17}, 513-519$

Ten Cate JM, Featherstone JD (1991): Mechanistic aspects of the interactions between fluoride and dental enamel. Crit Rev Oral Biol Med 2, 283-296

Tezel H, Ergucu Z, Onal B (2002): Effects of topical fluoride agents on artificial enamel lesion formation in vitro. Quintessence Int $\underline{33}, 347-352$.

Travess H, Roberts-Harry D, Sandy J (2004): Orthodontics. Part 6: Risks in orthodontic treatment. Br Dent J $\underline{196}, 71-77$

Tufekci E, Dixon JS, Gunsolley JC, Lindauer SJ (2011): Prevalence of white spot lesions during orthodontic treatment with fixed appliances. Angle Orthod. 81, 206-210

Tzeng D-Y, Berns RS (2005): A Review of principal component analysis and its applications to color technology. Color Res Appl $\underline{30}, 84-98$

Van der Burgt TP, Ten Bosch JJ, Borsboom PCF, Kortsmit WJPM (1990): A comparison of new and conventional methods for quantification of tooth color. J Prosthet Dent $\underline{63}, 155-162$ 
Vila Verde A, Ramos MM, Stoneham AM (2009): Benefits in cost and reduced discomfort of new techniques of minimally invasive cavity treatment. J Dent Res $\underline{88}, 297-299$

Watts A, Addy M (2001): Tooth discolouration and staining: a review of the literature. Br Dent $\mathrm{J} \underline{190}, 309-316$

Wiegand A, Köwing L, Attin T (2007): Impact of brushing force on abrasion of acid-softened and sound enamel. Arch Oral Biol $\underline{52}, 1043-1047$

Willmot DR (2004): White lesions after orthodontic treatment: does low fluoride make a difference? J Orthod. 31, 235-242,discussion 202

Zimmer BW, Rottwinkel Y (2004): Assessing patient-specific decalcification risk in fixed orthodontic treatment and ist impact on prophylactic procedures. Am J Orthod Dentofacial Orthop $\underline{126}, 318-324$ 


\section{Abkürzungsverzeichnis}

\begin{tabular}{|l|l|}
\hline AGS & angrenzender gesunder Schmelzbereich \\
\hline bzW. & beziehungsweise \\
\hline ca. & circa \\
\hline CIE & Commission internationale de l'éclairage \\
\hline Diff & Differenz \\
\hline F & weiblich \\
\hline FDI & Fédération Dentaire Internationale \\
\hline KFO & Kieferorthopädie \\
\hline KHN & knoop-hardness numbers \\
\hline M & männlich \\
\hline MB & Multibandapparatur, Multibracketapparatur \\
\hline n & Anzahl \\
\hline n.S. & nicht signifikant \\
\hline RI & Lichtbrechungsindex, Refractive Index \\
\hline S & Schmelz \\
\hline S. & Seite \\
\hline S. & Streptococcus \\
\hline SD & Standardabweichung, standard deviation \\
\hline SE & Standardfehler, standard error \\
\hline UMG & Universitätsmedizin Göttingen \\
\hline vs & versus \\
\hline WS & White-Spot \\
\hline WSL & White-Spot-Läsion \\
\hline z.B. & Zum Beispiel \\
\hline zW & zwischen \\
\hline
\end{tabular}




\section{Abbildungsverzeichnis}

\begin{tabular}{|c|c|c|}
\hline Abbildung 1a & Polarisationsmikroskopisches Bild einer Schmelzkaries & S. 7 \\
\hline Abbildung 1b & Histologische Einteilung der Approximalkaries & S. 8 \\
\hline Abbildung 2 & Patientenaufnahme mit schlechter Mundhygiene bei MB & S. 11 \\
\hline Abbildung 3 & Lichtbrechung Schmelz/WSL & S.16 \\
\hline $\begin{array}{l}\text { Abbildungen } 4 a \\
\text { und } 4 \mathrm{~b}\end{array}$ & $\begin{array}{l}\text { 4a: Icon-Patienten-Kit } \\
\text { 4b: und dessen Inhalt }\end{array}$ & S.18 \\
\hline Abbildung $5 a$ & $\begin{array}{l}\text { Das Spektrophotometer-Handmessgerät (ShadePilot, } \\
\text { Degudent, Hanau-Wolfgang, Deutschland) }\end{array}$ & S.20 \\
\hline Abbildung 5b & Position des Spektrophotometers bei der Messung & S.20 \\
\hline Abbildung 5c & $\begin{array}{l}\text { Bild von der Auswertung mit dem Shadepilot-Programm } \\
\text { (DeguDent Shadepilot, Hanau-Wolfgang, Deutschland) }\end{array}$ & S.21 \\
\hline Abbildung 6 & Dreidimensionale Darstellung des $L^{*} a^{*} b^{*}$-Systems & S.22 \\
\hline Abbildung 7 & $\begin{array}{l}\text { Die Farbbestimmung mithilfe eines standardisierten } \\
\text { Farbspiegels. }\end{array}$ & S.24 \\
\hline Abbildung 8 & Beispiel für die Quadrantenzuweisung & S.28 \\
\hline Abbildung 9a-h & $\begin{array}{l}\text { 9a/b: Legen der Retraktionsfäden } \\
\text { 9c: Anrauen mit Icon-Etch } \\
\text { 9d/e: Das Auftragen des Icon-Dry } \\
\text { 9f:Auftragen des Icon-Infiltrats } \\
\text { 9g: Lichtaushärtung } \\
\text { 9h:Abschlusssituation }\end{array}$ & S.30/31 \\
\hline Abbildung 10 & $\begin{array}{l}\text { Entwicklung der summierten Farb- und } \\
\text { Helligkeitsunterschiede zwischen den WSL und dem } \\
\text { umliegendem Schmelz }\end{array}$ & S. 36 \\
\hline Abbildung 11 & $\begin{array}{l}\text { Fallbeispiel. Einzelaufnahmen spektrophotometrisch } \\
\text { ausgewerteter Zähne zu den Zeitpunkten }\end{array}$ & S.38 \\
\hline Abbildung 12 & $\begin{array}{l}\text { Farb- und Helligkeitswerte }(\Delta \mathrm{E}-\text { Werte)-Vergleich der } \\
\text { behandelten Zähne im Vergleich zur Kontrollgruppe } \\
\text { zwischen Baseline (t0) und nach sechs Monaten (t5) }\end{array}$ & S.40 \\
\hline $\begin{array}{l}\text { Abbildungen } \\
\text { 13a-f }\end{array}$ & $\begin{array}{l}\text { Farb- und Helligkeitsveränderungen in den Gruppen } \\
\text { (Kontrollgruppe oder Icon) über die Zeit }\end{array}$ & $\begin{array}{l}\text { S. } 43,45 \text {, } \\
47\end{array}$ \\
\hline
\end{tabular}




\begin{tabular}{|l|l|c|}
\hline $\begin{array}{l}\text { Abbildungen } \\
\text { 14a-f }\end{array}$ & $\begin{array}{l}\text { Farb- und Helligkeitsveränderungen in den WSL und AGS } \\
\text { über die Zeit }\end{array}$ & $\begin{array}{c}\text { S. 44, 46, } \\
48\end{array}$ \\
\hline Abbildung 15 & $\Delta$ E-WSL/AGS-Werte der Icon-Gruppe nach 12 Monaten & S.52 \\
\hline Abbildung 16 & Fallbeispiel: verminderter Farbausgleich der WSL & S.58 \\
\hline
\end{tabular}

\section{Tabellenverzeichnis}

\begin{tabular}{|c|c|c|}
\hline Tabelle I & $\begin{array}{l}\text { Detaillierte Informationen und Zeiträume zu kieferorthopädischer } \\
\text { Behandlung, Beginn und Ablauf der Infiltrationsbehandlung und den } \\
\text { Folgeuntersuchungen. }\end{array}$ & S.32 \\
\hline Tabelle II & detaillierte Ergebnisse der Methodenfehleranalye & S.35 \\
\hline Tabelle III & $\begin{array}{l}\text { Inter-Gruppen-Vergleich: } \\
\text { die summierten } L^{*} / a^{*} / b^{*}-\text { Werte }(\Delta E) \text { zum Zeitpunkt Baseline (t0) } \\
\text { und nach sechs Monaten (t5) }\end{array}$ & S.37 \\
\hline Tabelle IV & $\begin{array}{l}\text { Der Intra-Gruppen-Vergleich der verschiedenen Werte von t0 bis t5 } \\
\text { für beide Gruppen. }\end{array}$ & S.39 \\
\hline Tabelle V & $\begin{array}{l}\text { Deskriptive Statistik } \\
\text { Entwicklung der Farb-und Helligkeitsunterschiede zwischen WSL } \\
\text { und AGS }\end{array}$ & S.41 \\
\hline Tabelle VI & $\begin{array}{l}\text { Deskriptive Statistik } \\
\text { Nachuntersuchung der infiltrierten Zähne nach } 12 \text { Monaten }\end{array}$ & S.50 \\
\hline Tabelle VII & $\begin{array}{l}\text { Entwicklung der Farb- und Helligkeitsunterschiede } \\
\text { Nachuntersuchung der infiltrierten Zähne nach } 12 \text { Monaten }\end{array}$ & S.51 \\
\hline Tabelle VIII & $\begin{array}{l}\text { Paarweise t-Tests } \\
\text { Nachuntersuchung der infiltrierten Zähne nach } 12 \text { Monaten }\end{array}$ & S.51 \\
\hline
\end{tabular}




\title{
12. Anhang
}

\section{Patientenaufklärungsbögen und Einverständniserklärungsformulare}

\author{
Georg-August-Universität \\ Universitätsklinikum $\approx$ Medizinische Fakultät \\ Bereich Humanmedizin $\approx$ D-37099 Göttingen \\ Zentrum für Zahn-, Mund- und Kieferheilkunde \\ Abt. Kieferorthopädie \\ Direktor: Prof. Dr. D. Kubein-Meesenburg \\ Hauptuntersucher: Priv.-Doz. Dr. M. Knösel \\ Tel. 0551-39-22017; mknoesel@yahoo.de \\ Abt. Kieferorthopädie, Robert-Koch-Str. 40
}

\section{Einwilligung volljähriger Probanden in die Teilnahme an der Klinischen Studie zur Wirksamkeit und Beurteilung möglicher Farbveränderungen von ,White-Spot- Läsionen' durch das Icon-Präparat}

Ich bin am von (Untersucher) über Art und Durchführung der minimalinvasiven Icon-Zahnversiegelungs-Therapie unterrichtet worden und habe das dazugehörige Informationsblatt gelesen. Weiterhin willige ich hiermit in die Aufnahme-Bedingung ein, während des Untersuchungszeitraumes auf zahnaufhellende, d.h. bleichende Maßnahmen (Verwendung von Bleaching-Gels und aufhellender Zahncremes) zu verzichten. Bei Problemen oder Beschwerden erreiche ich den Prüfarzt unter der o.g. Nummer. Über die darin beschriebene Anwendung des Füllungsverfahrens und die genannten Untersuchungsverfahren wurde ich in allem Umfang aufgeklärt. Ich hatte Gelegenheit, mich beim aufklärenden Prüfarzt über alle Fragen zu informieren und erhalte eine Kopie dieser Einwilligung. Ich habe zur Untersuchung im Rahmen der o.g. wissenschaflichen Studie und zur Durchführung keine weiteren Fragen und erkläre hiermit mein Einverständnis zu der besprochenen Untersuchung.

Das hier gegebene Einverständnis kann ich jederzeit, mündlich und -im Falle einer laufenden oder geplanten Behandlung-ohne medizinische Folgen für die Behandlung widerrufen.Ich erkläre mich einverstanden, dass die im Rahmen der durchgeführten Untersuchung ermittelten Daten in anonymisierter, d. h. nicht personenbezogener Form für wissenschaftliche Zwecke verwendet werden können.

Göttingen, den

(Unterschrift des Studienteilnehmers) 
Georg-August-Universität

Universitätsklinikum $\approx$ Medizinische Fakultät

Bereich Humanmedizin $\approx$ D-37099 Göttingen

Zentrum für Zahn-, Mund- und Kieferheilkunde

Abt. Kieferorthopädie

Direktor: Prof. Dr. D. Kubein-Meesenburg

Hauptuntersucher: Priv.-Doz. Dr. M. Knösel

Tel. 0551-39-22017; mknoesel@yahoo.de

Abt. Kieferorthopädie, Robert-Koch-Str. 40

\section{Einwilligung Erziehungsberechtigter minderjähriger Probanden in die Teilnahme an der Klinischen Studie zur Wirksamkeit und Beurteilung möglicher Farbveränderungen von ,White-Spot-Läsionen' durch das Icon-Präparat}

Ich bin am

von

(Untersucher) über Art und Durchführung der minimalinvasiven Icon-Zahnversiegelungs-Therapie unterrichtet worden und habe das dazugehörige Informationsblatt gelesen. Weiterhin willige ich hiermit in die Aufnahme-Bedingung ein, während des Untersuchungszeitraumes auf zahnaufhellende, d.h. bleichende Maßnahmen (Verwendung von Bleaching-Gels und aufhellender Zahncremes) zu verzichten. Bei Problemen oder Beschwerden erreiche ich den Prüfarzt unter der o.g. Nummer. Über die darin beschriebene Anwendung des Füllungsverfahrens und die genannten Untersuchungsverfahren wurde ich in allem Umfang aufgeklärt. Ich hatte Gelegenheit, mich beim aufklärenden Prüfarzt über alle Fragen zu informieren und erhalte eine Kopie dieser Einwilligung. Ich habe zur Untersuchung im Rahmen der o.g. wissenschaflichen Studie und zur Durchführung keine weiteren Fragen und erkläre hiermit mein Einverständnis zu der besprochenen Untersuchung an meinem Kind.

Das hier gegebene Einverständnis kann ich jederzeit, mündlich und -im Falle einer laufenden oder geplanten Behandlung-ohne medizinische Folgen für die

Behandlung widerrufen.Ich erkläre mich einverstanden, dass die im Rahmen der an meinem Kind durchgeführten Untersuchung ermittelten Daten in anonymisierter, d. h. nicht personenbezogener Form für wissenschaftliche Zwecke verwendet werden können.

Göttingen, den

(Unterschrift des Erziehungsberechtigten) 
Klinische Studie zur Wirksamkeit und Beurteilung möglicher Nebenwirkungen eines Präparates (ICON; DMG Chemisch-Pharmazeutische Fabrik GmbH, 22547 Hamburg, Germany) zur farblichen Angleichung inaktiver White-Spot-Läsionen an umgebende Schmelzareale

\section{Informationsblatt für volljährige Teilnehmer der Studie}

Sehr geehrte Interessentin, sehr geehrter Interessent unserer Studie! Uns sind an Ihren Zähnen Entkalkungsflecken, sog. White-Spot-Läsionen (WSL) aufgefallen. Diese sind nicht nur ästhetisch störend, sondern stellen darüber hinaus auch die Vorstufe einer Karies (Zahnfäule) dar: Bei ausbleibender Behandlung dieser WSL kann sich eine Karies manifestieren, die man später nur noch mit einer aufwändigen Füllungstherapie behandeln kann. Darüber hinaus stellen WSL, neben dem erhöhten Kariesrisiko, oft auch eine erhebliche ästhetische Beeinträchtigung dar, vor allem im gut sichtbaren Frontzahnbereich.

Wir möchten Ihnen daher die Teilnahme an unserer Studie vorschlagen, wobei wir Ihnen als Alternative zu einer herkömmlichen zahnärztlichen Füllungstherapie (,Bohren' mit anschließender Versiegelung mit einer Kunststoffüllung) den Zahn in einem schonenden Verfahren direkt versiegeln. Das Ziel ist es, den Zahn durch diese Versieglung zu schützen; als positiven Nebeneffekt gleicht sich die Farbe der hellen Flecken an den restlichen Zahn an. Mit gesundheitsbeeinträchtigenden Nebenwirkungen der angewandten Versiegelung ist, wie bei der herkömmlichen Füllungstherapie auch, nicht zu rechnen.

Zur Vorgehensweise:

Nach einer Farbbestimmung ihrer Zähne, werden diese vor der Behandlung gereinigt und die Zähne trocken gelegt, um während der Behandlung ein speichelfreies Arbeitsfeld zu erhalten. Anschließend wird ein Konditionierungsgel auf die Läsionsstellen der betroffenen Zähne auftragen. Nach 2 min Einwirkzeit wird es wieder abgespült und mit Luft getrocknet. Jetzt wird Icon-Dry, zur Trocknung, auftragen und nach 30 s Einwirkzeit mit Luft getrocknet. Zum Abschluss wird nun Icon-Infiltrant, ein flüssiger Kunststoff, auf den vorbereiteten Bereich aufgetragen 
und wirkt 3 min ein, bevor er für 40s lichtgehärtet wird. Der Vorgang wird ein zweites Mal wiederholt, diesmal mit einer Minute Einwirkzeit. Nach Beendigung der Behandlung wird erneut eine Farbbestimmung durchgeführt. Am Tag 2 (=nach 28 Tagen) wird die Farbe ein weiteres Mal ermittelt. Dies wird nach 12 Wochen (Tag 3) und 24 Wochen (Tag 4) noch einmal wiederholt.

Die genaue Durchführung der Behandlung wird Ihnen NOCH EINMAL beim ersten Termin gezeigt.

Zu wissenschaftlichen Zwecken werden die gemessenen Farb-Daten verschlüsselt gespeichert. Sie haben jederzeit das Recht, ohne Angabe von Gründen von der Teilnahme an der Studie zurückzutreten, ohne dass Ihnen daraus Nachteile in irgendeiner Form resultieren. 
Klinische Studie zur Wirksamkeit und Beurteilung möglicher Nebenwirkungen eines Präparates (ICON; DMG Chemisch-Pharmazeutische Fabrik GmbH, 22547 Hamburg, Germany) zur farblichen Angleichung inaktiver White-Spot-Läsionen an umgebende Schmelzareale

\section{Informationsblatt für minderjährige Teilnehmer der Studie}

Sehr geehrte Interessentin, sehr geehrter Interessent unserer Studie!

Uns sind an Deinen Zähnen Entkalkungsflecken, sog. White-Spot-Läsionen (WSL) aufgefallen. Diese sind nicht nur ästhetisch störend, sondern stellen darüber hinaus auch die Vorstufe einer Karies (Zahnfäule) dar: Bei ausbleibender Behandlung dieser WSL kann sich eine Karies manifestieren, die man später nur noch mit einer aufwändigen Füllungstherapie behandeln kann. Darüber hinaus stellen diese Entkalkungsflecken, neben dem erhöhten Kariesrisiko, oft auch eine erhebliche ästhetische Beeinträchtigung dar, vor allem im gut sichtbaren Frontzahnbereich.

Wir möchten Dir daher die Teilnahme an unserer Studie vorschlagen, wobei wir Dir als Alternative zu einer herkömmlichen zahnärztlichen Füllungstherapie (,Bohren' mit anschließender Versiegelung mit einer Kunststofffüllung) den Zahn in einem schonenden Verfahren direkt versiegeln, also verschließen. Das Ziel ist es, den Zahn durch diese Versieglung zu schützen; als positiven Nebeneffekt gleicht sich die Farbe der hellen Flecken an den restlichen Zahn an. Mit gesundheitsbeeinträchtigenden Nebenwirkungen der angewandten Versiegelung ist, wie bei der herkömmlichen Füllungstherapie auch, nicht zu rechnen.

Zur Vorgehensweise:

Nach einer Farbbestimmung ihrer Zähne, werden diese vor der Behandlung gereinigt und die Zähne trocken gelegt, um während der Behandlung ein speichelfreies Arbeitsfeld zu erhalten. Anschließend wird ein Konditionierungsgel auf die Läsionsstellen der betroffenen Zähne auftragen. Nach 2 min Einwirkzeit wird es wieder abgespült und mit Luft getrocknet. Jetzt wird Icon-Dry, zur Trocknung, auftragen und nach 30 s Einwirkzeit mit Luft getrocknet. Zum Abschluss wird nun 
Icon-Infiltrant, ein flüssiger Kunststoff, auf den vorbereiteten Bereich aufgetragen und wirkt 3 min ein, bevor er für 40s lichtgehärtet wird. Der Vorgang wird ein zweites Mal wiederholt, diesmal mit einer Minute Einwirkzeit. Nach Beendigung der Behandlung wird erneut eine Farbbestimmung durchgeführt. Am Tag 2 (=nach 28 Tagen) wird die Farbe ein weiteres Mal ermittelt. Dies wird nach 12 Wochen (Tag 3) und 24 Wochen (Tag 4) noch einmal wiederholt.

Die genaue Durchführung der Behandlung wird Dir NOCH EINMAL beim ersten Termin gezeigt.

Zu wissenschaftlichen Zwecken werden die gemessenen Farb-Daten verschlüsselt gespeichert. Du hast jederzeit das Recht, ohne Angabe von Gründen von der Teilnahme an der Studie zurückzutreten, ohne dass Dir daraus Nachteile in irgendeiner Form resultieren. 


\title{
Genehmigungsschreiben der Ethikkommission
}

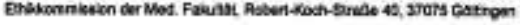

Herrn

PD Dr, med, dent. Michael Knosel

Abt. Kieferorthopadie

im Hause

\author{
UMG \\ Ethikkornmission \\ Vorsitzender: Prof. Dr. Wolfgang Poser \\ Referentin \\ Regienungsratitin Doris Wettschereck \\ 0551 / 39-8644 Tolefon \\ 37099 Gottingen Briofpost \\ Robert-Koch-Straße 40, 37075 Gottingen \\ Adresse \\ 0551/39-6629 Telefon \\ $0551 / 39-9536 \mathrm{Fax}$ \\ efhikemed uni-gosttingen.de E-Mail \\ www.ethikkammission.med.uni-goettingen.de \\ 24.02.2010 we-gó Datum
}

Nachrichtlich an: cand. Med. Amely Eckstein

\begin{abstract}
Antragsnummer:
4/8/09 (bitte stets angeben)

Studientitel:

Klinische Studie zur Wiksarnkeit und Beurteilung moglicher Neberwirkungen eines Präparates (ICON: DMG Chemisch-Pharmazeutische Fabrik GmbH. 22547 Hamburg. Germany ) zur farbilichen Angleichung inaktiver White-Spot-

Antragstellor: $\quad$ PD Dr. med, dent. Michaet KnOsel ,Abt. Keferorthopadie, UMG; Cand. med. dent Amely Eckstein
\end{abstract}

Sehr geehrter Herr PD, Dr. Knosel, sohr geehrte Damen und Herren,

wir bestatigen den Eingang thres Schreibens am 28.10.2009 und Ihrer email am 23.02.2010.

Folgende Unteriagen wurden vorgelegt:

- Geändertes Studienprotokoll

- Geànderte Erfassungsbogen

- Einwiligung far volljahrige Probanden

- Eirwilligungserklarung for die gesetzlichen Vertreter einschileß1. Minderjăhrige

- Patienteninformation for volliathrige und minderiahrige Probanden

Nach Erganzung der vorliegenden Dokumente und Beantwortung der im vorlaufigen Votum aufgefuhrten Fragen bestehen nunmehr keine ethischen und rechtichen Bedenken gegen die Durchfuhrung des oben genannten Forschungsworhabens.

Wir wonschen viel Erfolg bei der Durchfohrung thres Projektes.

Die Ethik-Kommission weist daraut hin, dass die ârztliche und juristische Verantwortung bei den jeweiligen Profarzten verbleibt.

Es wird bestatigt, dass Profarzte, die an der $0 . \mathrm{g}$. Studie beteiligt sind, nicht an der Abstimmung teilgenemman haben.

Die zustimmende Bewertung ist for die im Anhang aufgefohrten Personen/Einrichtungen guitig. Eine Anderung der lokalen Prafatelian ist der hiesigen Ethik-Kommission mitzuteilen.

Auf die Einhaltung einschisgiger Gesetze und Rechtsworschnitten wird hingewiesen. Die nach Rechtslage natwendigen Unterrichtungen (u. A. Profplanainderungen, entsprechende Zwischenfallsereignisse, neue Datanlage, Nachemeldung von Prutzentran, Abschlussbericht) sind des Ethk-Kommission unverzoglich worzulegen.

Die Ethik-Kommission bestatigt, dess sie aut Grundlage nationaler Gasetze, Vorschriften sowie der GCPNCH-Fichtlinie artellet.

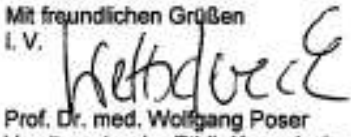

Vorsitzender der Ethik-Kommission

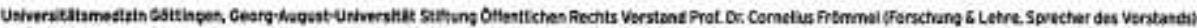

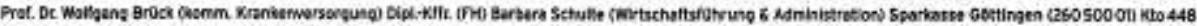


Die vorliegende Promotionsschrift war die Grundlage für folgende Veröffentlichung:

\section{Knösel M, Eckstein A, Helms H-J (2013):}

Durability of esthetic improvement following Icon resin infiltration of multibracket-induced white spot lesions compared with no therapy over 6 months: A single-center, split-mouth, randomized clinical trial.

Am J Orthod Dentofacial Orthop 144, 86-96 
Drittmittelunterstützung

Das in dieser Studie zur Behandlung der WSL verwendete Produkt Icon wurde von der Firma DMG (Icon, DMG Chemisch-Pharmazeutische Fabrik GmbH, 22547 Hamburg, Deutschland) zur Verfügung gestellt. Weiterhin wurde das Spektrophotometer-Handmessgerät (ShadePilot, Degudent, Hanau-Wolfgang, Deutschland), das zur Farb- und Helligkeitsmessung diente, von der Firma Degudent bereitgestellt. 


\section{Danksagung}

Mein größter Dank geht an Herrn PD Dr. Michael Knösel, der mir im Jahre 2009 freundlicherweise dieses Thema überließ. Eine umfassende, stets freundliche und perfekte Betreuung bei allen Fragen im Zusammenhang mit dieser Arbeit zeichneten inn aus.

Außerdem gilt mein Dank den Mitarbeitern der Kieferorthopädischen Abteilung der Universitätsmedizin Göttingen. Darunter möchte ich besonders Herrn Professor KubeinMeesenburg für die Möglichkeit, die Studie in seiner Abteilung durchführen zu können, danken. Desweiteren gilt mein Dank Herrn Dr. Frank Paschereit und seinem Team, die mir immer unterstützend standen.

Außerdem bedanke ich mich für die Hilfe in statistischen Fragen bei Herrn Dr. Hans-Joachim Helms und für die statistische Beratung im Rahmen der Doktorandensprechstunde bei der Abteilung Medizinische Statistik der Universität Göttingen. 